\title{
THE SINGULAR BIVARIATE QUARTIC TRACIAL MOMENT PROBLEM
}

\author{
ABHISHEK BHARDWAJ AND ALJAŽ ZALAR ${ }^{1}$
}

\begin{abstract}
The (classical) truncated moment problem, extensively studied by Curto and Fialkow, asks to characterize when a finite sequence of real numbers indexes by words in commuting variables can be represented with moments of a positive Borel measure $\mu$ on $\mathbb{R}^{n}$. In [BK12] Burgdorf and Klep introduced its tracial analog, the truncated tracial moment problem, which replaces commuting variables with non-commuting ones and moments of $\mu$ with tracial moments of matrices. In the bivariate quartic case, where indices run over words in two variables of degree at most four, every sequence with a positive definite $7 \times 7$ moment matrix $\mathcal{M}_{2}$ can be represented with tracial moments [BK10, BK12]. In this article the case of singular $\mathcal{M}_{2}$ is studied. For $\mathcal{M}_{2}$ of rank at most 5 the problem is solved completely; namely, concrete measures are obtained whenever they exist and the uniqueness question of the minimal measures is answered. For $\mathcal{M}_{2}$ of rank 6 the problem splits into four cases, in two of which it is equivalent to the feasibility problem of certain linear matrix inequalities. Finally, the question of a flat extension of the moment matrix $\mathcal{M}_{2}$ is addressed. While this is the most powerful tool for solving the classical case, it is shown here by examples that, while sufficient, flat extensions are mostly not a necessary condition for the existence of a measure in the tracial case.
\end{abstract}

\section{INTRODUCTION}

1.1. Context. The Moment problem (MP) is a classical question in analysis and concerns the existence of a positive Borel measure $\mu$ supported on a subset $K$ of $\mathbb{R}^{n}$, representing a given sequence of real or complex numbers indexed by monomials as the integration of the corresponding monomials w.r.t. $\mu$; nice expositions on the MP are [Akh65, KN77]. The solution to the MP on $\mathbb{R}^{n}$ is given by Haviland's theorem [Hav35], which establishes the duality with positive polynomials and relates the MP to real algebraic geometry (RAG). One of the cornerstones of RAG is the celebrated Schmüdgen theorem [Sch91], which solves the problem on compact basic closed semialgebraic sets and is the beginning of extensive research of the MP in RAG; we refer the reader to [Put93, PV99, DP01, PS01, KM02, Lau05, Lau09, Mar08, Las09] and the references therein for further details. Another important aspect of the MP is uniqueness of the representing measures. For compact sets the measure is unique (see e.g., [Mar08]), while for noncompact sets, the question of uniqueness is highly nontrivial (see [PS06, PS08]).

There are various generalizations of the MP. Functional analysis studies various versions of matrix and operator MPs; see [Kre49, Kov83, AV03, Vas03, BW11, CZ12, KW13] and references therein. The quantum MP from quantum physics is considered in [DLTW08]. The rational MP, which extends Schmüdgen theorem from the polynomial algebra to its localizations, is solved in [CMN11], while [GKM16] investigates the MP for the polynomial algebra in infinitely many variables. The MP on semialgebraic sets of generalized function is considered in [IKR14]. The beginning of free RAG is the solution of the full non-commutative (nc) MP by McCullough [McC01] and Helton [Hel02]. The nc MP has been further investigated in [HM04] and [HKM12]. In [HM04] the authors solve the full nc MP for nc matrix polynomials on a bounded nc semialgebraic set, while in [HKM12] the truncated nc MP for nc matrix polynomials on a convex nc semialgebraic set is solved. Finally, the most recent free MP is a tracial MP [BK10, BK12, BCKP13, BKP16] which is also the contents of this article.

The (classical) truncated moment problem (TMP) refers to the MP where only finitely many numbers in the sequence are given and one wants to know if they can be generated from a measure. By the Bayer-Teichmann version [BT06] of the Tchakaloff theorem [Tch57], it is sufficient to study only finitely atomic measures. Furthermore, the TMP is more general than the full MP by a result of Stochel [Sto01]. Curto and Fialkow shined new light on the TMP in their series of papers [CF96, CF98a, CF98b, CF02, CF04, CF05, CF08, Fia14]. One of their crowning achievements is the

Mathematical Sciences Institute, The Australian National University, Union Lane, Canberra ACT 2601

Institute of Mathematics, Physics, And MeChanics, JAdRAnska 19, 1000 LjublJana, SLOVENia

E-mail addresses: Abhishek. Bhardwaj@anu.edu.au, aljaz.zalareimfm.si.

Date: November 5, 2018.

2010 Mathematics Subject Classification. Primary 47A57, 15A45, 13J30; Secondary 11E25, 44A60, 15-04.

Key words and phrases. Truncated moment problem, non-commutative polynomial, moment matrix, affine linear transformations, flat extensions.

${ }^{1}$ Supported by the Slovenian Research Agency and in part by the Slovene Human Resources Development and Scholarship Fund. 
discovery that if a moment matrix admits a rank preserving extension (to a moment matrix), then the corresponding sequence admits a representing measure. Using this result, they completely solved the bivariate quartic TMP. Recently, the representing measures for the bivariate nonsingular quartic MP were constructed by the use of computer algebra [CS16].

The study of the truncated tracial moment problem (TTMP) was initiated by Burgdorf and Klep in [BK10, BK12], followed by [BCKP13]. A very nice reference including the results of this paragraph that also deals with polynomial optimization problems in matrix unknowns is [BKP16]. The motivation to study the TTMP comes from trace-positive polynomials, which are very interesting due to important applications, e.g., Connes' embedding problem [Con76] from functional analysis and Bessis-Moussa-Villani conjecture from statistical quantum mechanics have reformulations in terms of trace-positive polynomials [KS08a, KS08b, Bur11]. Determining if a polynomial is trace-positive, being the dual problem to the TTMP, is the connection of the TTMP with free RAG. Using this duality the bivariate quartic tracial MP with a positive definite moment matrix is solved in [BK10] (for alternative proof see [Caf13]) by showing that bivariate quartic trace-positive polynomials are always sums of hermitian squares and commutators. This fact does not generalize to higher powers or more variables (see examples in [KS08a, KS08b, Qua15]). In [BK12, BCKP13] the authors obtain the tracial analogs of the results on the classical moment problem of Curto and Fialkow, Stochel, Bayer and Teichmann, Fialkow and Nie [FN10], providing powerful means to tackle the special cases of the TTMP in a way analogous way to the classical one.

The origins of this paper lie in the first authors MSc thesis [Bha16] which contains alternative proofs for some preliminary results and some computational techniques to construct flat extensions of $\mathcal{M}_{2}$ to $\mathcal{M}_{3}$ in special cases.

In this section we state the main concepts and results of this paper. In Subsection 1.2 we introduce some essential definitions before stating the main results in Subsection 1.3. Finally, Subsection 1.4 is a guide to the organization of the rest of the paper.

1.2. Bivariate quartic tracial moment problem. In this subsection we state the main problem and introduce basic definitions used throughout this article.

1.2.1. Noncommutative bivariate polynomials. We denote by $\langle X, Y\rangle$ the free monoid generated by the noncommuting letters $X, Y$ and call its elements words in $X, Y$. Consider the free algebra $\mathbb{R}\langle X, Y\rangle$ of polynomials in $X, Y$ with coefficients in $\mathbb{R}$. Its elements are called noncommutative (nc) polynomials. Endow $\mathbb{R}\langle X, Y\rangle$ with the involution $p \mapsto p^{*}$ fixing $\mathbb{R} \cup\{X, Y\}$ pointwise. The length of the longest word in a polynomial $f \in \mathbb{R}\langle X, Y\rangle$ is the degree of $f$ and is denoted by $\operatorname{deg}(f)$ or $|f|$. We write $\mathbb{R}\langle X, Y\rangle_{\leq k}$ for all polynomials of degree at most $k$. For a word $w \in\langle X, Y\rangle$, $w^{*}$ is its reverse, and $v \in\langle X, Y\rangle$ is cyclically equivalent to $w$, which we denote by $v \stackrel{\text { cyc }}{\sim} w$, if and only if $v$ is a cyclic permutation of $w$.

1.2.2. Bivariate quartic real tracial moment problem. Given a sequence of real numbers $\beta \equiv \beta^{(4)}=\left(\beta_{w}\right)_{|w| \leq 4}$, indexed by words $w$ of length at most 4 such that

$$
\beta_{v}=\beta_{w} \quad \text { whenever } v \stackrel{\text { cyc }}{\sim} w \quad \text { and } \quad \beta_{w}=\beta_{w^{*}} \quad \text { for all }|w| \leq 4,
$$

i.e.,

$$
\begin{aligned}
\beta= & \left(\beta_{1}, \beta_{X}, \beta_{Y}, \beta_{X^{2}}, \beta_{X Y}=\beta_{Y X}, \beta_{Y^{2}}, \beta_{X^{3}}, \beta_{X^{2} Y}=\beta_{X Y X}=\beta_{Y X^{2}},\right. \\
& \beta_{X Y^{2}}=\beta_{Y X Y}=\beta_{Y^{2} X}, \beta_{Y^{3}}, \beta_{X^{4}}, \beta_{X^{3} Y}=\beta_{X^{2} Y X}=\beta_{X Y X^{2}}=\beta_{Y X^{3}}, \\
& \beta_{X^{2} Y^{2}}=\beta_{X Y^{2} X}=\beta_{Y^{2} X^{2}}=\beta_{Y X^{2} Y}, \beta_{X Y X Y}=\beta_{Y X Y X}, \\
& \left.\beta_{X Y^{3}}=\beta_{Y X Y^{2}}=\beta_{Y^{2} X Y}=\beta_{Y^{3} X}, \beta_{Y^{4}}\right),
\end{aligned}
$$

the bivariate quartic real tracial moment problem (BQTMP) for $\beta$ asks to find conditions for the existence of $N \in \mathbb{N}, t_{i} \in \mathbb{N}, \lambda_{i} \in \mathbb{R}_{>0}$ with $\sum_{i=1}^{N} \lambda_{i}=1$ and pairs of real symmetric matrices $\left(A_{i}, B_{i}\right) \in\left(\mathbb{S R}^{t_{i} \times t_{i}}\right)^{2}$, such that

$$
\beta_{w}=\sum_{i=1}^{N} \lambda_{i} \operatorname{Tr}\left(w\left(A_{i}, B_{i}\right)\right),
$$

where $w$ runs over the indices of the sequence $\beta$ and $\operatorname{Tr}$ denotes the normalized trace, i.e.,

$$
\operatorname{Tr}(A)=\frac{1}{t} \operatorname{tr}(A) \quad \text { for every } A \in \mathbb{R}^{t \times t} .
$$

If such data exist, we say that $\beta$ admits a representing measure. The vectors $\left(A_{i}, B_{i}\right)$ are atoms of size $t_{i}$ and the numbers $\lambda_{i}$ are densities. We say that $\mu$ is a representing measure of type $\left(m_{1}, m_{2}, \ldots, m_{r}\right)$ if it consists of exactly 
$m_{i} \in \mathbb{N} \cup\{0\}$ atoms of size $i$ and $m_{r} \neq 0$. A representing measure of type $\left(m_{1}^{(1)}, m_{2}^{(1)}, \ldots, m_{r_{1}}^{(1)}\right)$ is minimal, if there does not exist another representing measure of type $\left(m_{1}^{(2)}, m_{2}^{(2)}, \ldots, m_{r_{2}}^{(2)}\right)$ such that

$$
\begin{gathered}
r_{2}<r_{1} \quad \text { or } \quad\left(r_{2}=r_{1}, m_{r_{2}}^{(2)}<m_{r_{1}}^{(1)}\right) \quad \text { or } \quad\left(r_{2}=r_{1}, m_{r_{2}}^{(2)}=m_{r_{2}}^{(1)}, m_{r_{2}-1}^{(2)}<m_{r_{1}-1}^{(1)}\right) \\
\quad \text { or } \ldots \quad \text { or } \quad\left(r_{2}=r_{1}, m_{r_{2}}^{(2)}=m_{r_{2}}^{(1)}, \ldots, m_{2}^{(2)}=m_{2}^{(1)}, m_{1}^{(2)}<m_{1}^{(1)}\right) .
\end{gathered}
$$

We say that $\beta$ admits a noncommutative (nc) measure, if it admits a minimal meausre of type $\left(m_{1}, m_{2}, \ldots, m_{r}\right)$ with $r>1$. If $\beta_{1}=1$, then we say $\beta$ is normalized. We may always assume that $\beta$ is normalized (otherwise we replace Tr with $\frac{1}{\beta_{1}} \operatorname{Tr}$ ). If $\beta_{X^{2} Y^{2}}=\beta_{X Y X Y}$, we call $\beta$ a commutative (cm) sequence and the MP reduces to the classical one solved by Curto and Fialkow. If $\beta_{X^{2} Y^{2}} \neq \beta_{X Y X Y}$, then $\beta$ is a noncommutative (nc) sequence.

Remark 1.1. (1) Note that replacing a vector $\left(A_{i}, B_{i}\right)$ with any vector

$$
\left(U_{i} A_{i} U_{i}^{t}, U_{i} B_{i} U_{i}^{t}\right) \in\left(\mathbb{S R}^{t_{i} \times t_{i}}\right)^{2}
$$

where $U_{i} \in \mathbb{R}^{t_{i} \times t_{i}}$ is an orthogonal matrix, preserves (1.4).

(2) By the tracial version [BCKP13, Theorem 3.8] of Bayer-Teichmann theorem [BT06], the problem (1.4) is equivalent to the more general problem of finding a probability measure $\mu$ on $\left(\mathbb{S R}^{t \times t}\right)^{2}$ such that $\beta_{w}=$ $\int_{\left(\mathbb{S R}^{t \times t}\right)^{2}} \operatorname{Tr}(w(A, B)) \mathrm{d} \mu(A, B)$.

We associate to the sequence $\beta$ the truncated moment matrix $\mathcal{M}_{2}=\mathcal{M}_{2}(\beta)$ of order 2 with rows and columns indexed by words in $\mathbb{R}\langle X, Y\rangle_{\leq 2}$ in the degree-lexicographic order. The entry in row $U$ and column $V$ is $\beta_{U^{*} V}$, i.e.,

$$
\begin{array}{r}
\mathcal{M}_{2}= \\
\mathbb{1} \\
\mathbb{X} \\
\mathbb{Y} \\
\mathbb{X} \\
\mathbb{X} \\
\mathbb{X} \\
\mathbb{Y} \\
\mathbb{Y} \mathbb{X} \\
\mathbb{Y} \\
\mathbb{Y}^{2}
\end{array}
$$

Observe that the matrix $\mathcal{M}_{2}$ is symmetric. Let $S_{1}, S_{2}$ be subsets of $\left\{\mathbb{1}, \mathbb{X}, \mathbb{Y}, \mathbb{X}^{2}, \mathbb{X} \mathbb{Y}, \mathbb{Y} \mathbb{X}, \mathbb{Y}^{2}\right\}$. We will denote by $\left.\mathcal{M}_{2}\right|_{S_{1}, S_{2}}$ the submatrix of $\mathcal{M}_{2}$ consisting of the rows indexed by the elements of $S_{1}$ and the columns indexed by the elements of $S_{2}$. In case $S:=S_{1}=S_{2}$, we write $\left.\mathcal{M}_{2}\right|_{S}:=\left.\mathcal{M}_{2}\right|_{S, S}$ for short. If $\beta$ admits a measure, then $\mathcal{M}_{2}$ is positive semidefinite (psd); see Proposition 2.1. If $\mathcal{M}_{2}$ represents a $\mathrm{cm}$ sequence, we call it a $\mathbf{c m}$ moment matrix. Otherwise $\mathcal{M}_{2}$ is a nc moment matrix. By [BK12, Corollaries 3.19, 3.20], $\beta$ admits a measure if and only if there exists a moment matrix $\mathcal{M}_{2+k}$ extending $\mathcal{M}_{2}$, which admits a rank preserving extension $\mathcal{M}_{2+k+1}$. Furthermore, by [BK12, Corollary 3.2] in this case the atoms of size at most $\operatorname{rank}\left(\mathcal{M}_{2+k}\right)$ are sufficient. If $\mathcal{M}_{2}$ is positive definite, then $\beta$ admits a measure since all trace-positive polynomials of degree 4 are cyclically equivalent to sums of hermitian squares [BK10]. This is the duality established by [BK12, Theorem 4.4]. Moreover, the measure consists of at most 15 atoms of size 2 [BCKP13, Remark 3.9]. By dualizing a certain cone of trace-positive polynomials of degree 4 one obtains the so called completely positive semidefinite cone introduced by Laurent and Piovesan [LP15] which was used to study certain quantum graph parameters.

1.3. Results. In this paper we study the BQTMP for sequences with singular moment matrices. Initially, we approached this problem using a nc analog of the main tool for studying commutative sequences, i.e., finding rank preserving extensions of the moment matrices involved. As is already well established by Curto and Fialkow, the existence of a measure usually implies the existense of a rank preserving extension of a moment matrix, and hence a minimal measure with $\operatorname{rank}\left(\mathcal{M}_{2}\right)$ atoms; see Theorem 2.7 below. However, in stark contrast to the commutative case, our research soon revealed that this does not apply for noncommutative sequences $\beta$. Characterizing moment matrices $\mathcal{M}_{2}$ which admit a flat extension is insufficient for solving the BQTMP. The most versatile tool in tackling the BQTMP is the application of appropriate affine linear transformations on the sequences $\beta$, see Subsection 2.5 below. This splits the BQTMP into finitely many cases according to the column relations that exist in $\mathcal{M}_{2}$. For $\mathcal{M}_{2}$ of rank at most 5 , we completely characterize the existence, minimality and uniqueness of a measure in terms of the parameters $\beta_{w}$. In two out the four cases of $\mathcal{M}_{2}$ being of rank at most 6 , we prove that the BQTMP is equivalent to the feasibility problem of certain linear matrix inequalities with an additional rank-to-variety condition for one of them. In all but a single case - 
that of $\mathcal{M}_{2}$ being rank 6 and satisfying $\mathbb{Y}^{2}=\mathbb{1}$ - we show that atoms of size at most 2 suffice in the minimal measure of $\beta$. We now give a brief outline of the results we prove in this paper.

Outline of the results on BQTMP: (We assume that $\beta$ is a nc sequence.)

(1) If $\operatorname{rank}\left(\mathcal{M}_{2}\right) \leq 3$, then $\beta$ does not admit a nc measure. Namely, if $\beta$ admits a nc measure, then the columns $\mathbb{1}, \mathbb{X}, \mathbb{Y}, \mathbb{X} \mathbb{Y}$ in $\mathcal{M}_{2}$ must be linearly independent (see Corollary 2.3 below).

(2) If $\operatorname{rank}\left(\mathcal{M}_{2}\right)=4$, then it suffices, after applying an appropriate affine linear transformation, to study the case when $\mathcal{M}_{2}$ satisfies the column relations

$$
\mathbb{X}^{2}=\mathbb{1}, \quad \mathbb{X} \mathbb{Y}+\mathbb{Y} \mathbb{X}=a \mathbb{1}, \quad \mathbb{Y}^{2}=\mathbb{1},
$$

where $a \in(-2,2)$. By finding the representing atom of size 2 it turns out that such $\beta$ always admits a nc measure. Moreover, the representing atom is unique (up to orthogonal equivalence); see Theorem 3.1.

(3) If $\operatorname{rank}\left(\mathcal{M}_{2}\right)=5$, then it suffices, after applying an appropriate affine linear transformation, to study four special cases when $\mathcal{M}_{2}$ satisfies the column relation

$$
\mathbb{X}+\mathbb{Y} \mathbb{X}=\mathbf{0}
$$

and one of

$$
\mathbb{X}^{2}+\mathbb{Y}^{2}=\mathbb{1} \quad \text { or } \quad \mathbb{Y}^{2}=\mathbb{1} \quad \text { or } \quad \mathbb{Y}^{2}-\mathbb{X}^{2}=\mathbb{1} \quad \text { or } \quad \mathbb{Y}^{2}=\mathbb{X}^{2}
$$

(see Proposition 4.1 (1)). Due to the first relation the atoms in the minimal measure are of special form (see Proposition 5.1). In particular, all the atoms of size bigger than 1 have trace 0 . Analyzing moment matrices $\mathcal{M}_{2}$ generated only by atoms of size bigger than 1 with trace 0 , we show that only one such atom of size 2 is needed. Since every atom of size 2 generates a moment matrix of rank $4, \beta$ admits a nc measure if and only if there is a nc moment matrix $\widetilde{\mathcal{M}}_{2}$ of rank 4 representing a nc sequence $\widetilde{\beta}$ admitting a nc measure such that $\mathcal{M}_{2}-\alpha \widetilde{\mathcal{M}}_{2}, \alpha>0$, is a cm moment matrix admitting a measure. However, there are infinitely many possible atoms of size 2 satisfying relations (1.6) and (1.7). But there are at most 4 atoms of size 1 satisying (1.6) and (1.7). Therefore it is easier to subtract candidates for a cm moment matrices $\widehat{\mathcal{M}}_{2}$. Namely, we compute the smallest $\alpha>0$ such that $\operatorname{rank}\left(\mathcal{M}_{2}-\alpha \widehat{\mathcal{M}}_{2}\right)=4$. By the solution of the rank 4 case we can characterize exactly, in terms of the parameters $\beta_{w}$, when a nc measure exists, type of a minimal measure and its uniqueness.

(4) If $\operatorname{rank}\left(\mathcal{M}_{2}\right)=6$, then it suffices, after applying an appropriate affine linear transformation, to understand the four special cases when $\mathcal{M}_{2}$ satisfies one of the column relations

$$
\mathbb{X}^{2}+\mathbb{Y}^{2}=\mathbb{1} \quad \text { or } \quad \mathbb{X} \mathbb{Y}+\mathbb{Y} \mathbb{X}=\mathbf{0} \quad \text { or } \quad \mathbb{Y}^{2}-\mathbb{X}^{2}=\mathbb{1} \quad \text { or } \quad \mathbb{Y}^{2}=\mathbb{1}
$$

(see Proposition 4.1 (2)). In the first three cases the atoms in the minimal measure for $\beta$ are of size at most 2. In the first two cases the nc measure always exists if $\beta_{X}=\beta_{Y}=\beta_{X^{3}}=\beta_{X^{2} Y}=\beta_{Y^{3}}=0$. This is proved by computing the smallest $\alpha>0$ such that

$$
\operatorname{rank}\left(\mathcal{M}_{2}-\alpha\left(\mathcal{M}_{2}^{(1,0)}+\mathcal{M}_{2}^{(-1,0)}\right)\right)<6
$$

(resp. rank $\left(\mathcal{M}_{2}-\alpha \mathcal{M}_{2}^{(0,0)}\right)<6$ ), where $\mathcal{M}_{2}^{(x, y)}$ is the moment matrix generated by the atom $(x, y) \in \mathbb{R}^{2}$, and using the results of $\operatorname{rank}\left(\mathcal{M}_{2}\right) \leq 5$. Otherwise, still referring to the first two cases of rank 6 , if one of the moments $\beta_{X}, \beta_{Y}, \beta_{X^{3}}, \beta_{X^{2} Y}, \beta_{Y^{3}}$ is nonzero, then the existence of the nc measure is equivalent to the feasibilty of certain linear matrix inequalities.

1.4. Reader's guide. The paper is organized as follows. In Section 2 we prove some preliminary results about tracial moment sequences (see Subsections 2.1-2.5) and present the solution of the classical singular bivariate quartic MP (see Theorem 2.7). In Section 3 we solve BQTMP with $\mathcal{M}_{2}$ of rank 4. In Section 4 we reduce the study of BQTMP with $\mathcal{M}_{2}$ of rank 5 or 6 to four basic cases (see Proposition 4.1). In Section 5 we prove that in the basic cases of rank 5 and three basic cases of rank 6, atoms are of a special form and all the atoms of size at least 2 in some minimal measure have trace 0 (see Proposition 5.1). In Section 6 we solve all four basic cases of rank 5 (see Theorems 6.5, 6.8, 6.11, 6.14). In Section 7 we prove that in the first three basic cases of rank 6 atoms of size 2 suffice in the minimal measure for $\beta$. Then we study the relation $\mathbb{Y}^{2}=\mathbb{1}-\mathbb{X}^{2}$ in Subsection 7.1 (see Theorem 7.5 and Corollary 7.6) and the relation $\mathbb{X} \mathbb{Y}+\mathbb{Y} \mathbb{X}=\mathbf{0}$ in Subsection 7.2 (see Theorem 7.8 and Corollary 7.9). In Section 8 we analyze the existence of flat 
extensions with a moment structure $\mathcal{M}_{3}$ for moment matrices $\mathcal{M}_{2}$ of rank 6 .

Acknowledgement. Part of this paper was written at The University of Auckland under the supervision of Igor Klep who was the MSc supervisor of the first author and the PhD co-supervisor of the second author. Both authors wish to thank him for introducing us to this topic, the many insightful and inspiring discussions and support throughout the research. We are also thankful to two anonymous referees for useful comments and suggestions for improvements of the paper.

\section{PRELIMINARIES}

This section is devoted to terminology, notation and some preliminary results. Since these results hold for sequence of any degree (not only of degree 4) we will work with a general case.

2.1. Bivariate truncated tracial moment problem. BQTMP is a special case of a bivariate truncated tracial moment problem: Given a sequence of real numbers $\beta \equiv \beta^{(2 k)}=\left(\beta_{w}\right)_{|w| \leq 2 k}$, indexed by words $w$ of length at most $2 k$ such that

$$
\beta_{v}=\beta_{w} \quad \text { whenever } v \stackrel{\text { cyc }}{\sim} w \quad \text { and } \quad \beta_{w}=\beta_{w^{*}} \quad \text { for all }|w| \leq 2 k,
$$

when does there exist $N \in \mathbb{N}, t_{i} \in \mathbb{N}, \lambda_{i} \in \mathbb{R}_{>0}$ with $\sum_{i=1}^{N} \lambda_{i}=1$ and vectors $\left(A_{i}, B_{i}\right) \in\left(\mathbb{S R}^{t_{i} \times t_{i}}\right)^{2}$, such that

$$
\beta_{w}=\sum_{i=1}^{N} \lambda_{i} \operatorname{Tr}\left(w\left(A_{i}, B_{i}\right)\right)
$$

where $w$ runs over the indices of the sequence $\beta$.

2.2. Riesz functional and truncated moment matrix. For a polynomial $p \in \mathbb{R}\langle X, Y\rangle_{\leq 2 k}$, let $\hat{p}=\left(a_{w}\right)_{w}$ be its coefficient vector with respect to the lexicographically-ordered basis

$$
\left\{1, X, Y, X^{2}, X Y, Y X, Y^{2}, \ldots, X^{2 k}, \ldots, Y^{2 k}\right\}
$$

of $\mathbb{R}\langle X, Y\rangle_{\leq 2 k}$. Any sequence $\beta \equiv \beta^{(2 k)}: \beta_{1}, \ldots, \beta_{X^{2 k}}, \ldots, \beta_{Y^{2 k}}$, which satisfies (2.1) defines the Riesz functional $L_{\beta^{(2 k)}}: \mathbb{R}\langle X, Y\rangle_{\leq 2 k} \rightarrow \mathbb{R}$ by

$$
L_{\beta^{(2 k)}}(p):=\sum_{|w| \leq 2 k} a_{w} \beta_{w}, \quad \text { where } p=\sum_{|w| \leq 2 k} a_{w} w .
$$

Notice that

$$
\beta_{w}=L_{\beta^{(2 k)}}(w) \text { for every }|w| \leq 2 k .
$$

The truncated moment matrix $\mathcal{M}_{k}(\beta)$ of order $k$ is defined by

$$
\mathcal{M}_{k}=\mathcal{M}_{k}\left(\beta^{(2 k)}\right)=\left(\beta_{U^{*} V}\right)|U| \leq k,|V| \leq k,
$$

where the rows and columns are indexed by monomials in $\mathbb{R}\langle X, Y\rangle_{\leq k}$ in lexicographic order. When $k=2, \mathcal{M}_{2}$ is of the form (1.5). $\mathcal{M}_{k}$ is the unique matrix such that for $p, q \in \mathbb{R}\langle X, Y\rangle_{\leq k}$ we have that

$$
\left\langle\mathcal{M}_{k} \hat{p}, \hat{q}\right\rangle=L_{\beta^{(2 k)}}\left(p q^{*}\right),
$$

where $\langle\hat{p}, \hat{q}\rangle:=\hat{p}^{t} \hat{q}$. In particular, the row $w_{1}(X, Y)$ and column $w_{2}(X, Y)$ entry of $\mathcal{M}_{k}$ is equal to

$$
\left\langle\mathcal{M}_{k} \widehat{w_{2}(X, Y)}, \widehat{w_{1}(X, Y)}\right\rangle=L_{\beta(2 k)}\left(w_{2} w_{1}^{*}\right) .
$$

If $\beta^{(2 k)}$ admits a measure, i.e., (2.2) holds for every $\beta_{w}$, then for $p \in \mathbb{R}\langle X, Y\rangle$ of degree at most $k$ we have that

$$
\left\langle\mathcal{M}_{k} \hat{p}, \hat{p}\right\rangle=L_{\beta^{(2 k)}}\left(p p^{*}\right)=\sum_{i=1}^{m} \lambda_{i} \operatorname{Tr}\left(p\left(X_{i}, Y_{i}\right)\left(p\left(X_{i}, Y_{i}\right)\right)^{*}\right) \geq 0,
$$

where $\lambda_{i}, X_{i}, Y_{i}$ are as in (2.2). This proves the following proposition.

Proposition 2.1. If $\beta^{(2 k)}$ admits a measure, then $\mathcal{M}_{k}$ is positive semidefinite. 
2.3. Support of a measure and RG relations. Let $A$ be a matrix with its rows and columns indexed by words in $\mathbb{R}\langle X, Y\rangle$. Writing $w(\mathbb{X}, \mathbb{Y})$ we mean the column of $A$ indexed by the word $w$. $[A]_{E}$ denotes the compression of $A$ to the rows and columns indexed by the elements of the set $E$. Similarly for vectors $\mathbf{v}, \mathbf{v}_{E}$ denotes the compression of $\mathbf{v}$ to the entries indexed by words in $E . \mathbf{0}_{\mathbf{m} \times \mathbf{n}}$ stand for the $m \times n$ matrix with zero entries. Usually we will omit the subindex $m \times n$, when the size will be clear from the context.

Let $\mathcal{C}_{\mathcal{M}_{k}}$ denote the column space of $\mathcal{M}_{k}$, i.e.,

$$
\mathcal{C}_{\mathcal{M}_{k}}=\operatorname{span}\left\{\mathbb{1}, \mathbb{X}, \mathbb{Y}, \ldots, \mathbb{X}^{k} \ldots, \mathbb{Y}^{k}\right\} .
$$

For a polynomial $p \in \mathbb{R}\langle X, Y\rangle_{\leq k}$ of the form $p=\sum a_{w} w(X, Y)$, we define

$$
p(\mathbb{X}, \mathbb{Y})=\sum_{w} a_{w} w(\mathbb{X}, \mathbb{Y})
$$

and notice that $p(\mathbb{X}, \mathbb{Y}) \in \mathcal{C}_{\mathcal{M}_{k}}$. We express linear dependencies among the columns of $\mathcal{M}_{k}$ as

$$
p_{1}(\mathbb{X}, \mathbb{Y})=\mathbf{0}, \ldots, p_{m}(\mathbb{X}, \mathbb{Y})=\mathbf{0},
$$

for some $p_{1}, \ldots, p_{m} \in \mathbb{R}\langle X, Y\rangle_{\leq k}, m \in \mathbb{N}$. We define the free zero set $\mathcal{Z}(p)$ of $p \in \mathbb{R}\langle X, Y\rangle$ by

$$
\mathcal{Z}(p):=\left\{(A, B) \in\left(\mathbb{S R}^{t \times t}\right)^{2}: t \in \mathbb{N}, p(A, B)=\mathbf{0}_{t \times t}\right\} .
$$

Theorem 2.2 (1) (resp. (3)) is a real tracial analogue of [CF96, Proposition 3.1] (resp. [CF98b, Theorem 1.6]).

Theorem 2.2. Suppose $\beta^{(2 k)}$ admits a representing measure consisting of finitely many atoms $\left(X_{i}, Y_{i}\right) \in\left(\mathbb{S R}^{t_{i} \times t_{i}}\right)^{2}$, $t_{i} \in \mathbb{N}$, with the corresponding densities $\lambda_{i} \in(0,1)$. Let $p \in \mathbb{R}\langle X, Y\rangle_{\leq k}$ be a polynomial. Then the following are true:

(1) We have

$$
\bigcup_{i}\left(X_{i}, Y_{i}\right) \subseteq \mathcal{Z}(p) \quad \Leftrightarrow \quad p(\mathbb{X}, \mathbb{Y})=\mathbf{0} \text { in } \mathcal{M}_{k} .
$$

(2) Suppose the sequence $\beta^{(2 k+2)}=\left(\beta_{w}\right)_{|w| \leq k+1}$ is the extension of $\beta$ generated by

$$
\beta_{w}=\sum_{i} \lambda_{i} \operatorname{Tr}\left(w\left(X_{i}, Y_{i}\right)\right) .
$$

Let $\mathcal{M}_{k+1}$ be the corresponding moment matrix. Then:

$$
p(\mathbb{X}, \mathbb{Y})=\mathbf{0} \text { in } \mathcal{M}_{k} \Rightarrow p(\mathbb{X}, \mathbb{Y})=\mathbf{0} \text { in } \mathcal{M}_{k+1} .
$$

(3) (Recursive generation) For $q \in \mathbb{R}\langle X, Y\rangle_{\leq k}$ such that $p q \in \mathbb{R}\langle X, Y\rangle_{\leq k}$, we have

$$
p(\mathbb{X}, \mathbb{Y})=\mathbf{0} \text { in } \mathcal{M}_{k} \Rightarrow(p q)(\mathbb{X}, \mathbb{Y})=(q p)(\mathbb{X}, \mathbb{Y})=\mathbf{0} \text { in } \mathcal{M}_{k}
$$

Proof. Write $p=\sum_{|w| \leq n} a_{w} w$ where $a_{w} \in \mathbb{R}$. We have that

$$
\left\langle\mathcal{M}_{k} \hat{p}, \hat{p}\right\rangle=L_{\beta}\left(p p^{*}\right)=\sum_{|w|,|v| \leq k} a_{w} a_{v} \beta_{w v^{*}}=\sum_{i} \lambda_{i} \operatorname{Tr}\left(p\left(X_{i}, Y_{i}\right) p^{*}\left(X_{i}, Y_{i}\right)\right) .
$$

Observe that

$$
\mathcal{M}_{k} \hat{p}=p(\mathbb{X}, \mathbb{Y}) \text { in } \mathcal{M}_{k}
$$

Since $\mathcal{M}_{k}$ is psd,

$$
\mathcal{M}_{k} \hat{p}=\mathbf{0} \quad \Leftrightarrow \quad\left\langle\mathcal{M}_{k} \hat{p}, \hat{p}\right\rangle=0 .
$$

Since $p\left(X_{i}, Y_{i}\right) p^{*}\left(X_{i}, Y_{i}\right)$ is psd for each $i$, we have that

$$
\sum_{i} \lambda_{i} \operatorname{Tr}\left(p\left(X_{i}, Y_{i}\right) p^{*}\left(X_{i}, Y_{i}\right)\right)=0 \quad \Leftrightarrow \quad p\left(X_{i}, Y_{i}\right)=\mathbf{0}_{t_{i} \times t_{i}} .
$$

By (2.3)-(2.5), Theorem 2.2 (1) is true. Theorem 2.2 (2) follows easily.

It remains to prove Theorem 2.2 (3). If $\operatorname{deg}(p)=k$, then $q \in \mathbb{R}$ and statement is clear. Else $\operatorname{deg}(p)<k$. It suffices to prove that

$$
(X p)(\mathbb{X}, \mathbb{Y})=(p X)(\mathbb{X}, \mathbb{Y})=(Y p)(\mathbb{X}, \mathbb{Y})=(p Y)(\mathbb{X}, \mathbb{Y})=\mathbf{0} \text { in } \mathcal{M}_{k}
$$


First we will prove that $(X p)(\mathbb{X}, \mathbb{Y})=\mathbf{0}$ in $\mathcal{M}_{k}$. By Theorem 2.2 (1), we know that

$$
\begin{gathered}
\bigcup_{i}\left(X_{i}, Y_{i}\right) \subseteq \mathcal{Z}(p) \quad \Leftrightarrow \quad p(\mathbb{X}, \mathbb{Y})=\mathbf{0} \text { in } \mathcal{M}_{k}, \\
\bigcup_{i}\left(X_{i}, Y_{i}\right) \subseteq \mathcal{Z}(X p) \quad \Leftrightarrow \quad(X p)(\mathbb{X}, \mathbb{Y})=\mathbf{0} \text { in } \mathcal{M}_{k} .
\end{gathered}
$$

Since by assumption $p(\mathbb{X}, \mathbb{Y})=\mathbf{0}$ in $\mathcal{M}_{k}$, it follows by $\mathcal{Z}(p) \subseteq \mathcal{Z}(X p)$, (2.7) and (2.8) that

$$
(X p)(\mathbb{X}, \mathbb{Y})=\mathbf{0} \text { in } \mathcal{M}_{k} .
$$

By noticing that also the other three equalities of (2.6) are proved analogously, Theorem 2.2 (3) is true.

Column relations forced upon $\mathcal{M}_{k}$ with an application of Theorem 2.2 (3) will be important in solving BQTMP and we will refer to them as the RG relations. If $\mathcal{M}_{k}$ satisfies $\mathrm{RG}$ relations, we say $\mathcal{M}_{k}$ is recursively generated. The first consequence of the RG relations is the following important observation about a nc moment matrix $\mathcal{M}_{k}$.

Corollary 2.3. Suppose $k \geq 2$ and $\beta^{(2 k)}$ be a sequence such that $\beta_{X^{2} Y^{2}} \neq \beta_{X Y X Y}$. Then the columns $\mathbb{1}, \mathbb{X}, \mathbb{Y}, \mathbb{X} \mathbb{Y}$ of $\mathcal{M}_{k}$ are linearly independent.

Proof. Let us say that $\mathbf{0}=a \mathbb{1}+b \mathbb{X}+c \mathbb{Y}+d \mathbb{X} \mathbb{Y}$ for some $a, b, c, d \in \mathbb{R}$. If $d \neq 0$, then we have that $\beta_{X^{2} Y^{2}}=\beta_{X Y X Y}$, which is a contradiction with the assumption. Hence $d=0$. From $\mathbf{0}=a \mathbb{1}+b \mathbb{X}+c \mathbb{Y}$ it follows by the RG relations that

$$
\mathbf{0}=a \mathbb{X}+b \mathbb{X}^{2}+c \mathbb{X} \mathbb{Y}=a \mathbb{Y}+b \mathbb{X} \mathbb{Y}+c \mathbb{Y}^{2} .
$$

If $b \neq 0$ or $c \neq 0$, it follows that $\beta_{X^{2} Y^{2}}=\beta_{X Y X Y}$. Hence $b=c=0$. Finally $\mathbf{0}=a \mathbb{1}$ implies that $a=0$. This proves the corollary.

Corollary 2.4. Suppose $k \geq 2$ and $\beta^{(2 k)}$ be a sequence such that $\beta_{X^{2} Y^{2}} \neq \beta_{X Y X Y}$. If $\mathcal{M}_{k}$ is of rank at most 3 , then $\beta$ does not admit a measure.

2.4. Flat extensions. For a matrix $A \in \mathbb{S R}^{s \times s}$, an extension $\widetilde{A} \in \mathbb{S R}^{(s+u) \times(s+u)}$ of the form

$$
\widetilde{A}=\left(\begin{array}{cc}
A & B \\
B^{t} & C
\end{array}\right)
$$

for some $B \in \mathbb{R}^{s \times u}$ and $C \in \mathbb{R}^{u \times u}$, is called flat if $\operatorname{rank}(A)=\operatorname{rank}(\widetilde{A})$. This is equivalent to saying that there is a matrix $W \in \mathbb{R}^{s \times u}$ such that $B=A W$ and $C=W^{t} A W$. The connection between flat extensions and BTTMP is [BK12, Theorem 3.19].

Theorem 2.5. Let $\beta \equiv \beta^{(2 k)}$ be a sequence satisfying (2.1). If $\mathcal{M}_{k}(\beta)$ is psd and is a flat extension of $\mathcal{M}_{k-1}(\beta)$, then $\beta$ admits a representing measure.

2.5. Affine linear transformations. An important result for converting a given moment problem into a simpler, equivalent moment problem is the application of affine linear transformations to a sequence $\beta$. For $a, b, c, d, e, f \in \mathbb{R}$ with $b f-c e \neq 0$, let us define

$$
\phi(x, y)=\left(\phi_{1}(x, y), \phi_{2}(x, y)\right):=(a+b x+c y, d+e x+f y),(x, y) \in \mathbb{R}^{2} .
$$

Let $\widetilde{\beta}^{(2 k)}$ be the sequence obtained by the rule

$$
\widetilde{\beta}_{w}=L_{\beta^{(2 k)}}(w \circ \phi) \quad \text { for every }|w| \leq k .
$$

Notice that

$$
L_{\widetilde{\beta}(2 k)}(p)=L_{\beta^{(2 k)}}(p \circ \phi) \quad \text { for every } p \in \mathbb{R}\langle X, Y\rangle_{\leq k} .
$$

The following is the tracial analogue of [CF04, Proposition 1.9], which will allow us to make affine linear changes of variables.

Proposition 2.6. Suppose $\beta^{(2 k)}$ and $\widetilde{\beta}^{(2 k)}$ are as above and $\mathcal{M}_{k}$ and $\widetilde{\mathcal{M}}_{k}$ the corresponding moment matrices. Let $J_{\phi}: \mathbb{R}\langle X, Y\rangle_{\leq 2 k} \rightarrow \mathbb{R}\langle X, Y\rangle_{\leq 2 k}$ be the linear map given by

$$
J_{\phi} \widehat{p}:=\widehat{p \circ \phi} .
$$

Then the following hold:

(1) $\widetilde{\mathcal{M}}_{k}=\left(J_{\phi}\right)^{t} \mathcal{M}_{k} J_{\phi}$. 
(2) $J_{\phi}$ is invertible.

(3) $\widetilde{\mathcal{M}}_{k} \succeq 0 \Leftrightarrow \mathcal{M}_{k} \succeq 0$.

(4) $\operatorname{rank} \widetilde{\mathcal{M}}_{k}=\operatorname{rank} \mathcal{M}_{k}$.

(5) The formula $\mu=\tilde{\mu} \circ \phi$ establishes a one-to-one correspondence between the sets of representing measures of $\beta$ and $\tilde{\beta}$, and $\phi$ maps $\operatorname{supp}(\mu)$ bijectively onto $\operatorname{supp}(\tilde{\mu})$.

(6) $\mathcal{M}_{k}$ admits a flat extension if and only if $\widetilde{\mathcal{M}}_{k}$ admits a flat extension.

(7) For $p \in \mathbb{R}\langle X, Y\rangle_{\leq k}$, we have $p(\widetilde{X}, \widetilde{Y})=\left(J_{\phi}\right)^{t}((p \circ \phi)(X, Y))$.

Proof. The proof is the same to the proof of the corresponding statement in the commutative case [CF05, Proposition 1.9].

2.6. Classical bivariate quartic real moment problem. The classical bivariate quartic MP has been solved by Curto and Fialkow in a series of papers, e.g., [CF96, CF98a, CF98b, CF02, CF04, CF05, CF08, Fia14]. The main technique used was the analysis of the existence of a flat extension of a moment matrix $\mathcal{M}_{2}$. The solution of the singular bivariate quartic real MP is Theorem 2.7 below. Given a polynomial $p \in \mathbb{R}[x, y]_{\leq 2}$ we write $\mathcal{Z}_{c m}(p)=$ $\left\{(x, y) \in \mathbb{R}^{2}: p(x, y)=0\right\}$ for the variety generated by $p$.

Theorem 2.7. Suppose $\beta \equiv \beta^{(4)}$ is a commutative sequence with the associated moment matrix $\mathcal{M}_{2}$. Let

$$
\mathcal{V}:=\bigcap_{\substack{g \in \mathbb{R}[x, y]_{\leq 2} \\ g(\mathbb{X}, \mathbb{Y})=\mathbf{0}}} \mathcal{Z}_{c m}(g)
$$

be the variety associated to $\mathcal{M}_{2}$ and $p \in \mathbb{R}[x, y]$ a polynomial with $\operatorname{deg}(p)=2$. Then $\beta$ has a representing measure supported in $\mathcal{Z}_{c m}(p)$ if and only if $M(2)$ is positive semidefinite, recursively generated, satisfies $\operatorname{rank}(M(2)) \leq \operatorname{card} \mathcal{V}$ and has a column dependency relation $p(\mathbb{X}, \mathbb{Y})=0$.

Moreover, assume that $\mathcal{M}_{2}$ is positive semidefinite, recursively generated and satisfies the column dependency relation $p(\mathbb{X}, \mathbb{Y})$. The following statements are true:

(1) If $\operatorname{rank}\left(\mathcal{M}_{2}\right) \leq 3$, then $\mathcal{M}_{2}$ always admits a flat extension to a moment matrix $\mathcal{M}_{3}$ and hence $\beta$ admits a $\operatorname{rank}\left(\mathcal{M}_{2}\right)$-atomic minimal measure.

(2) If $\operatorname{rank}\left(\mathcal{M}_{2}\right)=4$, then $\beta$ does not necessarily come from a nc measure.

(3) If $\operatorname{rank}\left(\mathcal{M}_{2}\right)=5$, then $\beta$ always admits a nc measure, but $\mathcal{M}_{2}$ does not necessarily admit a flat extension to a moment matrix $\mathcal{M}_{3}$. There exists an affine linear transformation such that $\mathcal{V}$ is one of $x^{2}+y^{2}=1, y=x^{2}$, $x y=1, x^{2}=1$ or $x y=0$. In the first four cases $\mathcal{M}_{2}$ always admits a flat extension to a moment matrix $\mathcal{M}_{3}$ and hence $\beta$ admits a 5-atomic measure. However, in the last case there always exists a measure with 6 representing atoms, but not necessarily 5.

(4) If $\operatorname{rank}\left(\mathcal{M}_{2}\right)=6$, then $\mathcal{M}_{2}$ always admits a flat extension to a moment matrix $\mathcal{M}_{3}$ and hence $\beta$ admits a 6-atomic measure.

Proof. For the proof of the first part see [Fia14] and references therein. Let us now prove points (1)-(4) of the second part. Defining $z:=x+i y$ and $\bar{z}:=x-i y, \beta$ has a representing measure if and only if the complex sequence $\gamma_{i j}^{(4)}:=L_{\beta}\left(\bar{z}^{i} z^{j}\right)$ has a representing measure by [CF02, Proposition 1.12]. We write $\mathcal{M}_{2}^{\mathbb{C}}$ for the associated complex moment matrix. If $\mathbb{1}, \mathbb{X}, \mathbb{Y}$ are linearly dependent, then $\overline{\mathbb{Z}} \in \operatorname{span}\{\mathbb{1}, \mathbb{Z}\}$ in $\mathcal{M}_{2}^{\mathbb{C}}$ and hence $\mathcal{M}_{2}$ admits a flat extension to a moment matrix $\mathcal{M}_{3}$ by [CF98a, Theorem 2.1]. In particular, this is true if $\operatorname{rank}\left(\mathcal{M}_{2}\right) \leq 2$. If $\operatorname{rank}\left(\mathcal{M}_{2}\right)=3$ and $\mathbb{1}, \mathbb{X}, \mathbb{Y}$ are linearly independent, then $\mathbb{1}, \mathbb{Z}, \overline{\mathbb{Z}}$ are linearly independent, $\mathbb{Z} \overline{\mathbb{Z}} \in \operatorname{span}\{\mathbb{1}, \mathbb{Z}, \overline{\mathbb{Z}}\}$ and $\mathcal{M}_{2}$ admits a flat extension by [CF02, Theorem 1.1]. This proves (1). Parts (2) and (3) follow by the results in [CF02, Fia14]. Part (4) follows by [CS16, Theorem 2.1].

\section{SOLUTION OF THE BQTMP FOR $\mathcal{M}_{2}$ OF RANK 4}

In this section we solve the BQTMP for $\mathcal{M}_{2}$ of rank 4. In Theorem 3.1 we characterize exactly when the corresponding nc sequence $\beta$ admits a nc measure. Moreover, we prove that the minimal measure is unique (up to orthogonal equivalence) of type $(0,1)$ and find the concrete atom. In particular, $\beta$ admits a nc measure if and only if $\mathcal{M}_{2}$ admits a flat extension to a moment matrix $\mathcal{M}_{3}$.

Let $\beta \equiv \beta^{(4)}$ be a nc sequence such that the moment matrix $\mathcal{M}_{2} \equiv \mathcal{M}_{2}(\beta)$ has rank 4. By Corollary 2.3 we may assume that the set $\{\mathbb{1}, \mathbb{X}, \mathbb{Y}, \mathbb{X} \mathbb{Y}\}$ is linearly independent and hence a basis for the column space $\mathcal{C}_{\mathcal{M}_{2}}$. The main result of this section is the following. 
Theorem 3.1. Suppose $\beta \equiv \beta^{(4)}$ is a normalized nc sequence with the moment matrix $\mathcal{M}_{2}$ of rank 4 . Let the set $\{\mathbb{1}, \mathbb{X}, \mathbb{Y}, \mathbb{X} \mathbb{Y}\}$ be a basis for the column space $\mathcal{C}_{\mathcal{M}_{2}}$. Write

$$
\begin{aligned}
\mathbb{X}^{2} & =a_{1} \mathbb{1}+b_{1} \mathbb{X}+c_{1} \mathbb{Y}+d_{1} \mathbb{X} \mathbb{Y}, \\
\mathbb{Y} \mathbb{X} & =a_{2} \mathbb{1}+b_{2} \mathbb{X}+c_{2} \mathbb{Y}+d_{2} \mathbb{X} \mathbb{Y}, \\
\mathbb{Y}^{2} & =a_{3} \mathbb{1}+b_{3} \mathbb{X}+c_{3} \mathbb{Y}+d_{3} \mathbb{X} \mathbb{Y},
\end{aligned}
$$

where $a_{j}, b_{j}, c_{j}, d_{j} \in \mathbb{R}$ for $j=1,2,3$. The following statements are true:

(1) $d_{1}=d_{3}=0, d_{2}=-1$.

(2) $\beta$ admits a nc measure if and only if $\mathcal{M}_{2}$ has a flat extension to a moment matrix $\mathcal{M}_{3}$.

(3) $\beta$ admits a nc measure if and only if $\mathcal{M}_{2}$ is positive semidefinite and

$$
c_{1}=b_{3}=0, \quad b_{2}=c_{3}, \quad c_{2}=b_{1} .
$$

(4) Suppose $\beta$ admits a nc measure. Then the minimal measure is of type $(0,1)$ with a unique (up to orthogonal equivalence) atom $(X, Y) \in\left(\mathbb{S R}^{2 \times 2}\right)^{2}$ given by

$$
\begin{aligned}
& X=\left(\begin{array}{cc}
\sqrt{a_{1}+\frac{b_{1}^{2}}{4}} & 0 \\
0 & -\sqrt{a_{1}+\frac{b_{1}^{2}}{4}}
\end{array}\right)+\frac{b_{1}}{2} \cdot I_{2}, \\
& Y=\sqrt{a_{3}+\frac{c_{3}^{2}}{4}} \cdot\left(\begin{array}{cc}
\frac{a}{2} & \frac{1}{2} \sqrt{4-a^{2}} \\
\frac{1}{2} \sqrt{4-a^{2}} & -\frac{a}{2}
\end{array}\right)+\frac{c_{3}}{2} \cdot I_{2},
\end{aligned}
$$

where $a=\frac{4 a_{2}+2 b_{1} c_{3}}{\sqrt{\left(4 a_{1}+b_{1}^{2}\right)\left(4 a_{3}+c_{3}^{2}\right)}}$ and $I_{2}$ is the $2 \times 2$ identity matrix.

Proof. Part (1) follows by comparing the rows $\mathbb{X} \mathbb{Y}$ and $\mathbb{Y} \mathbb{X}$ on both sides of (3.1), (3.2), (3.3) and noticing that the columns $\mathbb{1}, \mathbb{X}, \mathbb{Y}, \mathbb{X}^{2}$ and $\mathbb{Y}^{2}$ have the same entries in the rows $\mathbb{X} \mathbb{Y}$ and $\mathbb{Y} \mathbb{X}$.

The implication $(\Leftarrow)$ of $(2)$ follows by Theorem 2.5. It remains to prove the converse. If $\beta$ admits a nc measure, then the extension $\mathcal{M}_{3}$ of $\mathcal{M}_{2}$, generated by the nc measure, must satisfy RG relations obtained from (3.1), (3.2), (3.3); see Theorem 2.2 (3). On multiplying (3.1) from right (resp. left) by $\mathbb{X}$ (resp. $\mathbb{Y}$ ) we conclude that in $\mathcal{M}_{3}$ the columns $\mathbb{X}^{3}, \mathbb{X}^{2} \mathbb{Y}, \mathbb{Y} \mathbb{X}^{2}$ lie in the linear span of the columns $\mathbb{1}, \mathbb{X}, \mathbb{Y}, \mathbb{X} \mathbb{Y}$. By analogous reasoning it follows from (3.3) that the same is true for $\mathbb{Y}^{3}, \mathbb{Y}^{2} \mathbb{X}, \mathbb{X} \mathbb{Y}^{2}$. Finally using these conclusions after multiplying (3.2) by $\mathbb{X}$ (resp. $\mathbb{Y}$ ), the same applies to $\mathbb{X} \mathbb{Y} \mathbb{X}, \mathbb{Y} \mathbb{X}$. Hence $\mathcal{M}_{3}$ is a flat extension of $\mathcal{M}_{2}$.

Now we prove the implication $(\Rightarrow)$ of (3). Let $\mathcal{M}_{3}$ be a flat extension of $\mathcal{M}_{2}$. Reasoning in the same way as in (2) we must have $\mathbb{X}^{3}=a_{1} \mathbb{X}+b_{1} \mathbb{X}^{2}+c_{1} \mathbb{X} \mathbb{Y}$ in the column space of $\mathcal{M}_{3}$. Since $\mathbb{X}^{3}$ has the same entries in rows $\mathbb{X} \mathbb{Y}, \mathbb{Y} \mathbb{X}$, it follows that $c_{1}=0$. Analogously we conclude that $b_{3}=0$. Applying an affine linear transformation $\phi_{1}(X, Y)=\left(X-\frac{b_{1}}{2}, Y-\frac{c_{3}}{2}\right)$ to $\beta$ we get $\widetilde{\beta}$ with a psd moment matrix $\widetilde{M}_{2}$ of rank 4 satisfying the relations

$$
\mathbb{X}^{2}=\left(a_{1}+\frac{b_{1}^{2}}{4}\right) \mathbb{1}, \quad \mathbb{X} \mathbb{Y}+\mathbb{Y} \mathbb{X}=a_{4} \mathbb{1}+b_{4} \mathbb{X}+c_{4} \mathbb{Y}, \quad \mathbb{Y}^{2}=\left(a_{3}+\frac{c_{3}^{2}}{4}\right) \mathbb{1},
$$

where

$$
a_{4}=a_{2}+\frac{b_{1} c_{3}}{2}, \quad b_{4}=b_{2}-c_{3}, \quad c_{4}=c_{2}-b_{1} .
$$

Claim 1: $a_{1}+\frac{b_{1}^{2}}{4}>0$ and $a_{3}+\frac{c_{3}^{2}}{4}>0$.

If $a_{1}+\frac{b_{1}^{2}}{4} \leq 0$, then $\widetilde{\beta}_{X^{4}}=\left(a_{1}+\frac{b_{1}^{2}}{4}\right) \widetilde{\beta}_{X^{2}} \leq 0$. The case $\widetilde{\beta}_{X^{4}}<0$ contradicts to $\widetilde{\mathcal{M}}_{2}$ being psd, while in the case $\widetilde{\beta}_{X^{4}}=0$ it follows that $\widetilde{\beta}_{X^{2}}=\widetilde{\beta}_{X^{4}}=\widetilde{\beta}_{X^{2} Y^{2}}=0$ which contradicts to the rank of $\widetilde{\mathcal{M}}_{2}$ being 4. Analogously we conclude that $a_{3}+\frac{c_{3}^{2}}{4}>0$.

Applying an affine linear transformation $\phi_{2}(X, Y)=\left(\frac{X}{\sqrt{a_{1}+\frac{b_{1}^{2}}{4}}}, \frac{Y}{\sqrt{a_{3}+\frac{c_{3}^{2}}{4}}}\right)$ to $\widetilde{\beta}$ we get $\widehat{\beta}$ with a psd moment matrix $\widehat{\mathcal{M}}_{2}$ of rank 4 satisfying the relations

$$
\mathbb{X}^{2}=\mathbb{1}, \quad \mathbb{X} \mathbb{Y}+\mathbb{Y} \mathbb{X}=a \mathbb{1}+b \mathbb{X}+c \mathbb{Y}, \quad \mathbb{Y}^{2}=\mathbb{1},
$$

where

$$
a=\frac{4 a_{2}+2 b_{1} c_{3}}{C}, \quad b=\frac{4\left(b_{2}-c_{3}\right)}{C}, \quad c=\frac{4\left(c_{2}-b_{1}\right)}{C},
$$


and $C=\sqrt{\left(4 a_{1}+b_{1}^{2}\right)\left(4 a_{3}+c_{3}^{2}\right)}$. By RG relations it follows from (3.5) that the extension $\widehat{\mathcal{M}}_{3}$ of $\widehat{\mathcal{M}}_{2}$ satisfies the relations

$$
\begin{aligned}
\mathbb{X}^{2} \mathbb{Y} & =\mathbb{Y}, & \mathbb{X}^{2} \mathbb{Y}+\mathbb{X} \mathbb{X} & =a \mathbb{X}+b \mathbb{X}^{2}+c \mathbb{X} \mathbb{Y} \\
\mathbb{X} \mathbb{Y}^{2}+\mathbb{Y} \mathbb{Y} & =a \mathbb{Y}+b \mathbb{X}+c \mathbb{Y}^{2}, & \mathbb{Y}^{2} \mathbb{X} & =\mathbb{X} .
\end{aligned}
$$

In particular,

$$
\mathbb{X}+\mathbb{Y} \mathbb{X}=a \mathbb{Y}+b \mathbb{X} \mathbb{Y}+c \mathbb{Y}^{2}, \quad \mathbb{Y}+\mathbb{X} \mathbb{Y}=a \mathbb{X}+b \mathbb{X}^{2}+c \mathbb{X} \mathbb{Y}
$$

Observing the rows $\mathbb{X} \mathbb{Y}$ and $\mathbb{Y} \mathbb{X}$ on the both sides of the equations in (3.7) and noticing that the columns $\mathbb{Y}, \mathbb{Y} \mathbb{X}, \mathbb{Y}^{2}$, $\mathbb{X}, \mathbb{X}^{2}$ have the same entries in the rows $\mathbb{X} \mathbb{Y}, \mathbb{Y} \mathbb{X}$, we must have $b=c=0$. Hence, (3.6) implies that $b_{2}=c_{3}$ and $c_{2}=b_{1}$.

Now we prove the implication $(\Leftarrow)$ of $(3)$. As above let

$$
\phi_{1}(X, Y)=\left(X-\frac{b_{1}}{2}, Y-\frac{c_{3}}{2}\right), \quad \phi_{2}(X, Y)=\left(\frac{X}{\sqrt{a_{1}+\frac{b_{1}^{2}}{4}}}, \frac{Y}{\sqrt{a_{3}+\frac{c_{3}^{2}}{4}}}\right) .
$$

Applying transformation $\phi_{2} \circ \phi_{1}$ to $\beta$ we get $\widehat{\beta}$ with a psd moment matrix $\widehat{\mathcal{M}}_{2}$ of rank 4 satisfying the relations

$$
\mathbb{X}^{2}=\mathbb{1}, \quad \mathbb{X} \mathbb{Y}+\mathbb{Y} \mathbb{X}=a \mathbb{1}, \quad \mathbb{Y}^{2}=\mathbb{1},
$$

where $a=\frac{4 a_{2}+2 b_{1} c_{3}}{\sqrt{\left(4 a_{1}+b_{1}^{2}\right)\left(4 a_{3}+c_{3}^{2}\right)}}$. We have to prove that $\widehat{\beta}$ admits a nc measure. From the relations (3.8) we get the following system

$$
\begin{aligned}
& \beta_{X^{2}}=1, \\
& 2 \beta_{X Y}=a, \\
& \beta_{Y^{2}}=1, \\
& \beta_{X^{3}}=\beta_{X}, \\
& 2 \beta_{X^{2} Y}=a \beta_{X}, \\
& \beta_{X Y^{2}}=\beta_{X} \text {, } \\
& \beta_{X^{2} Y}=\beta_{Y}, \\
& 2 \beta_{X Y^{2}}=a \beta_{Y}, \\
& \beta_{Y^{3}}=\beta_{Y}, \\
& \beta_{X^{4}}=\beta_{X^{2}}, \\
& 2 \beta_{X^{3} Y}=a \beta_{X^{2}} \text {, } \\
& \beta_{X^{2} Y^{2}}=\beta_{X^{2}} \text {, } \\
& \beta_{X^{3} Y}=\beta_{X Y}, \\
& \beta_{X^{2} Y^{2}}+\beta_{X Y X Y}=a \beta_{X Y}, \\
& \beta_{X Y^{3}}=\beta_{X Y}, \\
& \beta_{X^{2} Y^{2}}=\beta_{Y^{2}} \text {, } \\
& 2 \beta_{X Y^{3}}=a \beta_{Y^{2}}, \\
& \beta_{Y^{4}}=\beta_{Y^{2}} \text {. }
\end{aligned}
$$

The solutions to (3.9) are given by

$$
\begin{aligned}
\beta_{Y^{4}}=\beta_{X^{4}}=\beta_{X^{2} Y^{2}}=\beta_{Y^{2}}=\beta_{X^{2}} & =1 \\
\beta_{X Y^{3}}=\beta_{X^{3} Y}=\beta_{X Y} & =\frac{a}{2}, \\
\beta_{X Y X Y} & =\frac{a^{2}}{2}-1,
\end{aligned}
$$

and one of the following:

Case 1: $\beta_{X Y^{2}}=\beta_{X^{2} Y}=\beta_{Y^{3}}=\beta_{Y}=\beta_{X^{3}}=\beta_{X}=0$,

Case 2: $a=2$ and $\beta_{X^{3}}=\beta_{X}=\beta_{Y}=\beta_{Y^{3}}=\beta_{X Y^{2}}=\beta_{X^{2} Y} \in \mathbb{R}$,

Case 3: $a=-2$ and $\beta_{X^{3}}=\beta_{X}=-\beta_{Y}=-\beta_{Y^{3}}=\beta_{X Y^{2}}=-\beta_{X^{2} Y} \in \mathbb{R}$.

However, in Cases 2 and 3 the submatrices $\left[\widehat{\mathcal{M}}_{2}\right]_{\{\mathbb{X}, \mathbb{Y}\}}$ are of the form $\left(\begin{array}{cc}1 & \pm 1 \\ \pm 1 & 1\end{array}\right)$ and are not positive definite. Hence we are in Case 1 and $\widehat{\mathcal{M}}_{2}$ takes the form

$$
\widehat{\mathcal{M}}_{2}=\left(\begin{array}{ccccccc}
1 & 0 & 0 & 1 & \frac{a}{2} & \frac{a}{2} & 1 \\
0 & 1 & \frac{a}{2} & 0 & 0 & 0 & 0 \\
0 & \frac{a}{2} & 1 & 0 & 0 & 0 & 0 \\
1 & 0 & 0 & 1 & \frac{a}{2} & \frac{a}{2} & 1 \\
\frac{a}{2} & 0 & 0 & \frac{a}{2} & 1 & \frac{a^{2}}{2}-1 & \frac{a}{2} \\
\frac{a}{2} & 0 & 0 & \frac{a}{2} & \frac{a^{2}}{2}-1 & 1 & \frac{a}{2} \\
1 & 0 & 0 & 1 & \frac{a}{2} & \frac{a}{2} & 1
\end{array}\right) .
$$

$\widehat{\mathcal{M}}_{2}$ is psd if and only if $a \in(-2,2)$. Now notice that the representing atom $(\widehat{X}, \widehat{Y})$ for $\widehat{\mathcal{M}}_{2}$ is given by the pair

$$
\widehat{X}=\left(\begin{array}{cc}
1 & 0 \\
0 & -1
\end{array}\right), \quad \widehat{Y}=\left(\begin{array}{cc}
\frac{a}{2} & -\frac{1}{2} \sqrt{4-a^{2}} \\
-\frac{1}{2} \sqrt{4-a^{2}} & -\frac{a}{2}
\end{array}\right) .
$$


This proves the implication $(\Leftarrow)$ of $(3)$.

It remains to prove part (4). Let $\phi_{1}, \phi_{2}$ and $\widehat{\mathcal{M}}_{2}$ be as in the proof of part (3). By Proposition 2.6 (5) the measures $\mu$ for $\beta$ are in the bijective correspodence with the measures $\widehat{\mu}$ for $\widehat{\beta}$ given by the rule $\mu=\widehat{\mu}\left(\phi_{2} \circ \phi_{1}\right)$ and $\operatorname{supp}(\mu)=$ $\left(\phi_{2} \circ \phi_{1}\right)^{-1}(\operatorname{supp}(\widehat{\mu}))$. We have that

$$
\left(\phi_{2} \circ \phi_{1}\right)^{-1}(X, Y)=\left(\sqrt{a_{1}+\frac{b_{1}^{2}}{4}} \cdot X+\frac{b_{1}}{2}, \sqrt{a_{3}+\frac{c_{3}^{2}}{4}} \cdot Y+\frac{c_{3}}{2}\right) .
$$

Therefore it suffices to prove the following claim.

Claim 2: The atom $(\widehat{X}, \widehat{Y}) \in\left(\mathbb{S R}^{2 \times 2}\right)^{2}$ of the form (3.11) is up to orthogonal equivalence the unique atom for the measure $\widehat{\mu}$ of $\widehat{\beta}$.

Let $(X, Y) \in\left(\mathbb{S R}^{2 \times 2}\right)^{2}$ be an atom representing $\widehat{\beta}$. Since $X, Y$ do not commute and $X^{2}=I_{2}$, the eigenvalues of $X$ must be $1,-1$. Hence we may assume (after conjugating by a suitable orthogonal matrix) that

$$
X=\widehat{X} \quad \text { and } \quad Y=\left(\begin{array}{cc}
k_{1} & k_{2} \\
k_{2} & -k_{1}
\end{array}\right) \quad \text { for some } k_{1}, k_{2} \in \mathbb{R} .
$$

The equality $Y^{2}=I_{2}$ implies that $k_{1}^{2}+k_{2}^{2}=1$, while from $\operatorname{Tr}(X Y)=\frac{a}{2}$ we get that $k_{1}=\frac{a}{2}$ and hence $k_{2}= \pm \frac{1}{2} \sqrt{4-a^{2}}$. It is now easy to check that the moment matrix $\mathcal{M}_{2}^{(X, Y)}$ generated by $(X, Y)$ where $\left(k_{1}, k_{2}\right)=$ $\left(\frac{a}{2}, \pm \frac{1}{2} \sqrt{4-a^{2}}\right)$ is equal to $\widehat{\mathcal{M}}_{2}$. Since both solutions are unitarily equivalent pairs (the orthogonal equivalence acts on the standard basis vectors $e_{1}, e_{2}$ as $e_{1} \mapsto e_{1}, e_{2} \mapsto-e_{2}$ ), this proves Claim 2.

The following corollary will be very important in the proofs of theorems about the existence of a nc measure in the rank 5 case.

Corollary 3.2. Suppose $\beta \equiv \beta^{(4)}$ has a psd moment matrix $\mathcal{M}_{2}$ of rank 4 satisfying the relations

$$
\mathbb{X}^{2}=a \mathbb{1}, \quad \mathbb{X} \mathbb{Y}+\mathbb{Y} \mathbb{X}=b \mathbb{1}, \quad \mathbb{Y}^{2}=c \mathbb{1} \quad \text { for some } a, b>0, c \in \mathbb{R} .
$$

Then

$$
\beta_{X}=\beta_{Y}=\beta_{X^{3}}=\beta_{X^{2} Y}=\beta_{X Y^{2}}=\beta_{Y^{3}}=0 .
$$

Proof. Applying an affine linear transformation $\phi(X, Y)=\left(\frac{X}{\sqrt{a}}, \frac{Y}{\sqrt{c}}\right)$ to $\mathcal{M}_{2}$ the relations of the corresponding matrix $\widetilde{M}_{2}$ become

$$
X^{2}=\mathbb{1}, \quad \mathbb{X} \mathbb{Y}+\mathbb{Y} \mathbb{X}=\frac{b}{\sqrt{a c}} \mathbb{1}, \quad \mathbb{Y}^{2}=\mathbb{1} .
$$

Then $\widetilde{M}_{2}$ is of the form (3.10) (where we replace $a$ with $\frac{b}{\sqrt{a c}}$ ). In particular, we have

$$
\widetilde{\beta}_{X}=\widetilde{\beta}_{Y}=\widetilde{\beta}_{X^{3}}=\widetilde{\beta}_{X^{2} Y}=\widetilde{\beta}_{X Y^{2}}=\widetilde{\beta}_{Y^{3}}=0 .
$$

Since the moments $\beta_{w}$ and $\widetilde{\beta}_{w}$ for $|w| \leq 4$ are scalar multiples of each other, (3.12) follows from (3.13).

\section{RANKS 5 AND 6 - REDUCTIONS}

In this section we establish an essential result for solving a BQTMP with a moment matrix of rank 5 or 6 . Namely, it suffices to solve the BQTMP only for moment matrices satisfying especially nice column relations; see Proposition 4.1. In the subsequent sections we will analyze each of those cases separately.

Proposition 4.1. Suppose a nc sequence $\beta \equiv \beta^{(4)}$ has a moment matrix $\mathcal{M}_{2}$ of rank 5 or 6 . Let $L_{\beta}$ be the Riesz functional belonging to $\beta$. If $\beta$ admits a nc measure, then there exists an affine linear transformation $\phi$ such that a sequence $\widehat{\beta}$, given by $\widehat{\beta}_{w}=L_{\beta}(w \circ \phi)$ for every $|w(X, Y)| \leq 4$, has a moment matrix $\widehat{\mathcal{M}}_{2}$ such that:

(1) If $\mathcal{M}_{2}$ is of rank 5 , then $\widehat{\mathcal{M}}_{2}$ satisfies $\mathbb{X} \mathbb{Y}+\mathbb{Y} \mathbb{X}=\mathbf{0}$ and one of the following relations:

Basic case 1: $\mathbb{X}^{2}+\mathbb{Y}^{2}=\mathbb{1}$,

Basic case 2: $\mathbb{Y}^{2}=\mathbb{1}$,

Basic case 3: $\mathbb{Y}^{2}-\mathbb{X}^{2}=\mathbb{1}$,

Basic case $4: \mathbb{Y}^{2}=\mathbb{X}^{2}$.

(2) If $\mathcal{M}_{2}$ is of rank 6 , then $\widehat{\widehat{\mathcal{M}}}_{2}$ satisfies one of the following relations:

Basic relation 1: $\mathbb{Y}^{2}=\mathbb{1}-\mathbb{X}^{2}$, 
Basic relation 2: $\mathbb{Y}^{2}=\mathbb{1}+\mathbb{X}^{2}$,

Basic relation 3: $\mathbb{X} \mathbb{Y}+\mathbb{Y} \mathbb{X}=\mathbf{0}$,

Basic relation 4: $\mathbb{Y}^{2}=\mathbb{1}$.

To prove Proposition 4.1 we need some lemmas.

Lemma 4.2. Suppose a nc sequence $\beta \equiv \beta^{(4)}$ has a moment matrix $\mathcal{M}_{2}$ of rank 5 or 6 satisfying the relation

$$
\mathbb{Y}^{2}=a_{1} \mathbb{1}+a_{2} \mathbb{X}+a_{3} \mathbb{Y}+a_{4} \mathbb{X}^{2}+a_{5} \mathbb{X} \mathbb{Y}+a_{6} \mathbb{Y} \mathbb{X}
$$

where $a_{i} \in \mathbb{R}$ for each $i$. Let $L_{\beta}$ be the Riesz funtional belonging to $\beta$. If $\beta$ admits a nc measure, then there exists an affine linear transformation $\phi$ of the form

$$
\phi(X, Y)=\left(\alpha_{1} X+\alpha_{2}, \alpha_{3} Y+\alpha_{4}\right),
$$

where $\alpha_{i} \in \mathbb{R}$ for each $i, \alpha_{1} \neq 0, \alpha_{4} \neq 0$, such that the sequence $\widehat{\beta}$ given by $\widehat{\beta}_{w}=L_{\beta}(w \circ \phi)$ for every $|w(X, Y)| \leq 4$, has a moment matrix $\widehat{\mathcal{M}}(2)$ satisfying one of the following relations:

Relation 1: $\mathbb{Y}^{2}=\mathbb{1}-\mathbb{X}^{2}$,

Relation 2: $\mathbb{Y}^{2}=\mathbb{1}$,

Relation 3: $\mathbb{Y}^{2}=\mathbb{1}+\mathbb{X}^{2}$,

Relation 4: $\mathbb{Y}^{2}=\mathbb{X}^{2}$.

Moreover, the relation 4 is equivalent to the relation

Relation $4: \mathbb{X} \mathbb{Y}+\mathbb{Y} \mathbb{X}=\mathbf{0}$.

Proof. By comparing the rows $\mathbb{X} \mathbb{Y}, \mathbb{Y} \mathbb{X}$ on both sides of (4.1) we conclude that $a_{5}=a_{6}$. We rewrite the relation (4.1) as

$$
\left(\mathbb{Y}-a_{5} \mathbb{X}\right)^{2}=a_{1} \mathbb{1}+a_{2} \mathbb{X}+a_{3} \mathbb{Y}+\left(a_{4}+a_{5}^{2}\right) \mathbb{X}^{2} .
$$

Applying an affine linear transformation $\phi_{1}(X, Y)=\left(X, Y-a_{5} X\right)$ to $\beta$ we get $\widetilde{\beta}$ with the moment matrix $\widetilde{\mathcal{M}}(2)$ satisfying the relation

$$
\mathbb{Y}^{2}=a_{1} \mathbb{1}+\left(a_{2}+a_{3} a_{5}\right) \mathbb{X}+a_{3} \mathbb{Y}+a_{4} \mathbb{X}^{2} .
$$

We separate three possibilities according to the sign of $a_{4} \in \mathbb{R}$.

Case 1: $a_{4}<0$. The relation (4.3) can be rewritten as

$$
\left(\mathbb{Y}-\frac{a_{3}}{2}\right)^{2}=-\left(\sqrt{\left|a_{4}\right|} \mathbb{X}-\frac{a_{2}+a_{3} a_{5}}{2 \sqrt{\left|a_{4}\right|}}\right)^{2}+\left(a_{1}+\frac{a_{3}^{2}}{4}+\frac{\left(a_{2}+a_{3} a_{5}\right)^{2}}{4 a_{4}}\right) \mathbb{1} .
$$

Applying an affine linear transformation $\phi_{2}(X, Y)=\left(\sqrt{\left|a_{4}\right|} X-\frac{a_{2}+a_{3} a_{5}}{2 \sqrt{\left|a_{4}\right|}}, Y-\frac{a_{3}}{2}\right)$ to $\widetilde{\beta}$ we get $\bar{\beta}$ with $\overline{\mathcal{M}}(2)$ satisfying the relation

$$
\mathbb{Y}^{2}=-\mathbb{X}^{2}+\left(a_{1}+\frac{a_{3}^{2}}{4}+\frac{\left(a_{2}+a_{3} a_{5}\right)^{2}}{4 a_{4}}\right) \mathbb{1}
$$

If $C_{1}:=a_{1}+\frac{a_{3}^{2}}{4}+\frac{\left(a_{2}+a_{3} a_{5}\right)^{2}}{4 a_{4}} \leq 0$, then by comparing the row $\mathbb{Y}^{2}$ on both sides of (4.4) we get

$$
0 \leq \beta_{Y^{4}}+\beta_{X^{2} Y^{2}}=C_{1} \cdot \beta_{Y^{2}} \leq 0
$$

where we used that $\beta_{Y^{4}} \geq 0, \beta_{X^{2} Y^{2}} \geq 0, \beta_{Y^{2}} \geq 0$. But then $\beta_{Y^{4}}=\beta_{X^{2} Y^{2}}=\beta_{Y^{2}}=0$, which contradicts to the rank of $\widetilde{\mathcal{M}}(2)$ being 5 or 6 . Therefore $C_{1}>0$. Applying an affine linear transformation $\phi_{3}(X, Y)=\left(\frac{X}{\sqrt{C_{1}}}, \frac{Y}{\sqrt{C_{1}}}\right)$ to $\bar{\beta}$ we get $\widehat{\beta}$ with $\widehat{\mathcal{M}}(2)$ satisfying

which is the relation 1 .

$$
\mathbb{Y}^{2}=\mathbb{1}-\mathbb{X}^{2}
$$

Case 2: $a_{4}=0$. Multiplying (4.3) with $\mathbb{Y}$ we get

$$
\mathbb{Y}^{3}=a_{1} \mathbb{Y}+\left(a_{2}+a_{3} a_{5}\right) \mathbb{X} \mathbb{Y}+a_{3} \mathbb{Y}^{2} .
$$

By comparing the rows $\mathbb{X} \mathbb{Y}, \mathbb{Y} \mathbb{X}$ on both sides of (4.5) we conclude that $a_{2}+a_{3} a_{5}=0$. We can rewrite (4.3) as

$$
\left(\mathbb{Y}-\frac{a_{3}}{2}\right)^{2}=\left(a_{1}+\frac{a_{3}^{2}}{4}\right) \mathbb{1}
$$


Applying an affine linear transformation $\phi_{4}(X, Y)=\left(X, Y-\frac{a_{3}}{2}\right)$ to $\widetilde{\beta}$ we get $\bar{\beta}$ with $\overline{\mathcal{M}}(2)$ satisfying

$$
\mathbb{Y}^{2}=\left(a_{1}+\frac{a_{3}^{2}}{4}\right) \mathbb{1}
$$

If $C_{2}:=a_{1}+\frac{a_{3}^{2}}{4} \leq 0$, then by comparing the row $\mathbb{Y}^{2}$ on both sides of (4.6) we get

$$
0 \leq \beta_{Y^{4}}=\left(a_{2}+\frac{c_{2}^{2}}{4}\right) \beta_{Y^{2}} \leq 0,
$$

where we used that $\beta_{Y^{4}} \geq 0, \beta_{Y^{2}} \geq 0$. But then $\beta_{Y^{4}}=\beta_{Y^{2}}=0$ and hence also $\beta_{X^{2} Y^{2}}=0$, which contradicts to the rank of $\widetilde{\mathcal{M}}(2)$ being 5 or 6 . Therefore $C_{2}>0$. Applying an affine linear transformation $\phi_{5}(X, Y)=\left(X, \frac{Y}{\sqrt{C_{2}}}\right)$ to $\bar{\beta}$ we get $\widehat{\beta}$ with $\widehat{\mathcal{M}}(2)$ satisfying

which is the relation 2 .

$$
\mathbb{Y}^{2}=\mathbb{1}
$$

Case 3: $a_{4}>0$. The relation (4.3) can be rewritten as

$$
\left(\mathbb{Y}-\frac{a_{3}}{2}\right)^{2}=\left(\sqrt{a_{4}} \mathbb{X}+\frac{a_{2}+a_{3} a_{5}}{2 \sqrt{a_{4}}}\right)^{2}+\left(a_{1}+\frac{a_{3}^{2}}{4}-\frac{\left(a_{2}+a_{3} a_{5}\right)^{2}}{4 a_{4}}\right) \mathbb{1}
$$

Applying an affine linear transformation $\phi_{6}(X, Y)=\left(\sqrt{a_{4}} X+\frac{a_{2}+a_{3} a_{5}}{2 \sqrt{a_{4}}}, Y-\frac{a_{3}}{2}\right)$ to $\widetilde{\beta}$ we get $\bar{\beta}$ with $\overline{\mathcal{M}}(2)$ satisfying

$$
\mathbb{Y}^{2}=\mathbb{X}^{2}+\left(a_{1}+\frac{a_{3}^{2}}{4}-\frac{\left(a_{2}+a_{3} a_{5}\right)^{2}}{4 a_{4}}\right) \mathbb{1}
$$

We separate three possibilities according to the sign of $C_{3}:=a_{1}+\frac{a_{3}^{2}}{4}-\frac{\left(a_{2}+a_{3} a_{5}\right)^{2}}{4 a_{4}}$.

Case 3.1: $C_{3}>0$. Applying an affine linear transformation $\phi_{7}(X, Y)=\left(\frac{X}{\sqrt{C_{3}}}, \frac{Y}{\sqrt{C_{3}}}\right)$ to $\bar{\beta}$ we get $\widehat{\beta}$ with $\widehat{\mathcal{M}}(2)$ satisfying

which is the relation 3 .

$$
\mathbb{Y}^{2}=\mathbb{1}+\mathbb{X}^{2}
$$

Case 3.2: $C_{3}=0$. The relation (4.7) is

$$
\mathbb{Y}^{2}=\mathbb{X}^{2}
$$

which is the relation 4. Applying an affine linear transformation $\phi_{8}(X, Y)=(X-Y, X+Y)$ to $\widetilde{\beta}$ we get $\bar{\beta}$ with $\overline{\mathcal{M}}(2)$ satisfying

which is the relation 4'.

$$
\mathbb{X} \mathbb{Y}+\mathbb{Y} \mathbb{X}=\mathbf{0}
$$

Case 3.3: $C_{3}<0$. Applying an affine linear transformation $\phi_{9}(X, Y)=(Y, X)$ to $\bar{\beta}$ we come into Case 3.1.

Lemma 4.3. Suppose a nc sequence $\beta \equiv \beta^{(4)}$ has a moment matrix $\mathcal{M}_{2}$ of rank 5 with linearly independent columns $\mathbb{1}, \mathbb{X}, \mathbb{Y}, \mathbb{X} \mathbb{Y}$. Then one of the following cases occurs:

Case 1: The set $\{\mathbb{1}, \mathbb{X}, \mathbb{Y}, \mathbb{X} \mathbb{Y}, \mathbb{Y}\}$ is the basis for $\mathcal{C}_{\mathcal{M}_{2}}$ and the columns $\mathbb{X}^{2}, \mathbb{Y}^{2}$ belong to the $\operatorname{span}\{\mathbb{1}, \mathbb{X}, \mathbb{Y}\}$.

Case 2: The set $\left\{\mathbb{1}, \mathbb{X}, \mathbb{Y}, \mathbb{X}^{2}, \mathbb{X} \mathbb{Y}\right\}$ is the basis for $\mathcal{C}_{\mathcal{M}_{2}}$.

Case 3: The set $\left\{\mathbb{1}, \mathbb{X}, \mathbb{Y}, \mathbb{Y}^{2}, \mathbb{Y} \mathbb{X}\right\}$ is the basis for $\mathcal{C}_{\mathcal{M}_{2}}$.

Proof. If $\mathbb{X}^{2} \notin \operatorname{span}\{\mathbb{1}, \mathbb{X}, \mathbb{Y}\}$, it follows by comparing the rows $\mathbb{X} \mathbb{Y}$ and $\mathbb{Y} \mathbb{X}$ that $\mathbb{X}^{2} \notin \operatorname{span}\{\mathbb{1}, \mathbb{X}, \mathbb{Y}, \mathbb{X} \mathbb{Y}\}$. Hence we are in Case 2. Similarly, if $\mathbb{Y}^{2} \notin \operatorname{span}\{\mathbb{1}, \mathbb{X}, \mathbb{Y}\}$, then we are in Case 3. Otherwise $\left\{\mathbb{X}^{2}, \mathbb{Y}^{2}\right\} \subseteq \operatorname{span}\{\mathbb{1}, \mathbb{X}, \mathbb{Y}\}$ and since $\mathcal{M}_{2}$ is of rank $5,\{\mathbb{1}, \mathbb{X}, \mathbb{Y}, \mathbb{X} \mathbb{Y}, \mathbb{Y} \mathbb{X}\}$ is a basis for $\mathcal{C}_{\mathcal{M}_{2}}$. Hence we are in Case 1 .

Lemma 4.4. Suppose a nc sequence $\beta \equiv \beta^{(4)}$ has a moment matrix $\mathcal{M}_{2}$ of rank 6 with linearly independent columns $\mathbb{1}, \mathbb{X}, \mathbb{Y}, \mathbb{X} \mathbb{Y}$. There exists an affine linear transformation $\phi$ such that a sequence $\widehat{\beta}$, given by $\widehat{\beta}_{w}=L_{\beta}(w \circ \phi)$ for every $|w(X, Y)| \leq 4$, has a moment matrix $\widehat{\mathcal{M}}_{2}$ such that:

Case 1: The set $\left\{\mathbb{1}, \mathbb{X}, \mathbb{Y}, \mathbb{X}^{2}, \mathbb{X} \mathbb{Y}, \mathbb{Y}\right\}$ is the basis for $\mathcal{C}_{\widehat{\mathcal{M}}_{2}}$.

Case 2: The set $\left\{\mathbb{1}, \mathbb{X}, \mathbb{Y}, \mathbb{X}^{2}, \mathbb{X} \mathbb{Y}, \mathbb{Y}^{2}\right\}$ is the basis for $\mathcal{C}_{\widehat{\mathcal{M}}_{2}}$. 
Proof. If $\mathbb{Y}^{2} \in \operatorname{span}\left\{\mathbb{1}, \mathbb{X}, \mathbb{Y}, \mathbb{X}^{2}, \mathbb{X} \mathbb{Y}, \mathbb{Y}\right\}$, then we are in Case 1 (linear independence of the columns $\{\mathbb{1}, \mathbb{X}, \mathbb{Y}, \mathbb{X} 2, \mathbb{X} \mathbb{Y}, \mathbb{Y}\}$ follows from the rank of $\mathcal{M}_{2}$ being 6). Otherwise $\mathbb{Y}^{2} \notin \operatorname{span}\left\{\mathbb{1}, \mathbb{X}, \mathbb{Y}, \mathbb{X}^{2}, \mathbb{X} \mathbb{Y}, \mathbb{Y} \mathbb{X}\right\}$. In particular, $\left\{\mathbb{1}, \mathbb{X}, \mathbb{Y}, \mathbb{X} \mathbb{Y}, \mathbb{Y}^{2}\right\}$ is a linearly independent set. Now we have two possibilities. Either $\left\{\mathbb{1}, \mathbb{X}, \mathbb{Y}, \mathbb{X} \mathbb{Y}, \mathbb{Y}^{2}, \mathbb{X}^{2}\right\}$ is a linearly independent set and we are in Case 2 , or $\mathbb{X}^{2} \in \operatorname{span}\left\{\mathbb{1}, \mathbb{X}, \mathbb{Y}, \mathbb{X} \mathbb{Y}, \mathbb{Y}^{2}\right\}$ and $\left\{\mathbb{1}, \mathbb{X}, \mathbb{Y}, \mathbb{X} \mathbb{Y}, \mathbb{Y}^{2}, \mathbb{Y} \mathbb{X}\right\}$ is a linearly independent set. After applying an affine linear transformation $\phi(X, Y)=(Y, X)$ we are in Case 1.

Lemma 4.5. Suppose a nc sequence $\beta \equiv \beta^{(4)}$ has a moment matrix $\mathcal{M}_{2}$ satisfying one of the relations

$$
\mathbb{Y}^{2}+\mathbb{X}^{2}=\mathbb{1} \text { or } \quad \mathbb{Y}^{2}-\mathbb{X}^{2}=\mathbb{1} \text { or } \quad \mathbb{Y}^{2}=\mathbb{X}^{2}
$$

If $\beta$ admits a nc measure $\mu$, then the extension $\mathcal{M}_{3}:=\left(\begin{array}{cc}\mathcal{M}_{2} & B_{3} \\ B_{3}^{t} & C_{3}\end{array}\right)$ generated by $\mu$ satisfies the relations

$$
\mathbb{X}^{2} \mathbb{Y}=\mathbb{Y} \mathbb{X}^{2} \text { and } \mathbb{X} \mathbb{Y}^{2}=\mathbb{Y}^{2} \mathbb{X} \text {. }
$$

In particular, the rows $\mathbb{X} \mathbb{Y}, \mathbb{Y} \mathbb{X}$ are the same in the columns $\mathbb{X}^{2} \mathbb{Y}, \mathbb{Y} \mathbb{X}^{2}$ and the columns $\mathbb{X} \mathbb{Y}^{2}, \mathbb{Y}^{2} \mathbb{X}$.

Proof. We will give the proof in the case of the relation $\mathbb{Y}^{2}+\mathbb{X}^{2}=\mathbb{1}$. The other two cases are proved in the same way. Multiplying $\mathbb{Y}^{2}+\mathbb{X}^{2}=\mathbb{1}$ by $\mathbb{Y}\left(\right.$ resp. $\mathbb{X}$ ) from the left (resp. right) gives $\mathbb{X}^{2} \mathbb{Y}=-\mathbb{Y}^{3}+\mathbb{Y}=\mathbb{Y}^{2}$ and $\mathbb{Y}^{2} \mathbb{X}=\mathbb{X}-\mathbb{X}^{3}=\mathbb{X} \mathbb{Y}^{2}$. By Theorem 2.2 we must have $\mathbb{X}^{2} \mathbb{Y}=\mathbb{Y}^{2}$ and $\mathbb{X} \mathbb{Y}^{2}=\mathbb{Y}^{2} \mathbb{X}$ in $\mathcal{M}_{3}$.

Finally we give the proof of Proposition 4.1 (1).

Proof of Proposition 4.1 (1). By Proposition 2.3 the columns $\mathbb{1}, \mathbb{X}, \mathbb{Y}, \mathbb{X} \mathbb{Y}$ of $\mathcal{M}_{2}$ are linearly independent. By Lemma 4.3 there are three cases to consider.

Case 1: The set $\{\mathbb{1}, \mathbb{X}, \mathbb{Y}, \mathbb{X} \mathbb{Y}, \mathbb{Y}\}$ is the basis for $\mathcal{C}_{\mathcal{M}_{2}}$ and the columns $\mathbb{X}^{2}, \mathbb{Y}^{2}$ belong to the $\operatorname{span}\{\mathbb{1}, \mathbb{X}, \mathbb{Y}\}$.

By assumption there are constants $a_{j}, b_{j}, c_{j} \in \mathbb{R}$ for $j=1,2$ such that

$$
\mathbb{X}^{2}=a_{1} \mathbb{1}+b_{1} \mathbb{X}+c_{1} \mathbb{Y} \quad \text { and } \quad \mathbb{Y}^{2}=a_{2} \mathbb{1}+b_{2} \mathbb{X}+c_{2} \mathbb{Y} .
$$

By multiplying the first relation with $\mathbb{X}$ and the second with $\mathbb{Y}$ it follows that if $\beta$ admits a nc measure, then $c_{1}=b_{2}=0$. Let

$$
\phi_{1}(X, Y)=\left(X-\frac{b_{1}}{2}, Y-\frac{c_{3}}{2}\right), \quad \phi_{2}(X, Y)=\left(\frac{X}{\sqrt{a_{1}+\frac{b_{1}^{2}}{4}}}, \frac{Y}{\sqrt{a_{3}+\frac{c_{3}^{2}}{4}}}\right) .
$$

Applying an affine linear transformation $\phi_{2} \circ \phi_{1}$ to $\beta$ we get $\widetilde{\beta}$ with $\widetilde{M}(2)$ satisfying

$$
\mathbb{X}^{2}=\mathbb{Y}^{2}=\mathbb{1}
$$

Equivalently, the relations are

$$
\mathbb{Y}^{2}-\mathbb{X}^{2}=\mathbf{0}, \quad \mathbb{Y}^{2}=\mathbb{1} .
$$

Finally applying an affine linear transformation $\phi_{3}(X, Y)=\left(\frac{X+Y}{2}, \frac{Y-X}{2}\right)$ to $\widetilde{\beta}$ we get $\widehat{\beta}$ with $\widehat{\mathcal{M}}(2)$ satisfying

$$
\mathbb{X} \mathbb{Y}+\mathbb{Y}=\mathbf{X}, \quad \mathbb{X}^{2}+\mathbb{Y}^{2}=\mathbb{1}
$$

Hence we are in a basic case 1 of Proposition 4.1.

Case 2: The set $\left\{\mathbb{1}, \mathbb{X}, \mathbb{Y}, \mathbb{X}^{2}, \mathbb{X} \mathbb{Y}\right\}$ is the basis for $\mathcal{C}_{\mathcal{M}_{2}}$.

By assumption there are constants $a_{j}, b_{j}, c_{j}, d_{j}, e_{j} \in \mathbb{R}$ for $j=1,2$ such that

$$
\mathbb{Y} \mathbb{X}=a_{1} \mathbb{1}+b_{1} \mathbb{X}+c_{1} \mathbb{Y}+d_{1} \mathbb{X}^{2}+e_{1} \mathbb{X} \mathbb{Y}, \quad \mathbb{Y}^{2}=a_{2} \mathbb{1}+b_{2} \mathbb{X}+c_{2} \mathbb{Y}+d_{2} \mathbb{X}^{2}+e_{2} \mathbb{X} \mathbb{Y} .
$$

By comparing the rows $\mathbb{X} \mathbb{Y}, \mathbb{Y} \mathbb{X}$ of the both sides of equations we conclude that $e_{1}=-1$ and $e_{2}=0$, so that the relation are

$$
\mathbb{X} \mathbb{Y}+\mathbb{Y} \mathbb{X}=a_{1} \mathbb{1}+b_{1} \mathbb{X}+c_{1} \mathbb{Y}+d_{1} \mathbb{X}^{2} \quad \text { and } \quad \mathbb{Y}^{2}=a_{2} \mathbb{1}+b_{2} \mathbb{X}+c_{2} \mathbb{Y}+d_{2} \mathbb{X}^{2} .
$$

By Lemma 4.2 there exists an affine linear transformation $\phi_{4}$ of the form (4.2) such that after applying $\phi_{4}$ to $\beta$ the second relation in (4.8) of the corresponding matrix $\overline{\mathcal{M}}(2)$ becomes one of the following:

$$
\mathbb{Y}^{2}=\mathbb{1} \quad \text { or } \quad \mathbb{Y}^{2}=\mathbb{1}-\mathbb{X}^{2} \quad \text { or } \quad \mathbb{Y}^{2}=\mathbb{X}^{2} \quad \text { or } \quad \mathbb{Y}^{2}=\mathbb{1}+\mathbb{X}^{2},
$$


while the first relation in (4.8) becomes

$$
\mathbb{X} \mathbb{Y}+\mathbb{Y} \mathbb{X}=a_{3} \mathbb{1}+b_{3} \mathbb{X}+c_{3} \mathbb{Y}+d_{3} \mathbb{X}^{2}
$$

where $a_{3}, b_{3}, c_{3}, d_{3} \in \mathbb{R}$. We separate four possibilities according to the relation in (4.9).

Case 2.1: $\mathbb{Y}^{2}=\mathbb{1}$ in (4.9). The relation (4.10) can be rewritten in the form

$$
\mathbb{Y}\left(\mathbb{X}-\frac{c_{3}}{2}\right)+\left(\mathbb{X}-\frac{c_{3}}{2}\right) \mathbb{Y}=a_{3} \mathbb{1}+b_{3} \mathbb{X}+d_{3} \mathbb{X}^{2}
$$

Applying an affine linear transformation $\phi_{5}(X, Y)=\left(X-\frac{c_{3}}{2}, Y\right)$ to $\bar{\beta}$ we get $\breve{\beta}$ with $\breve{\mathcal{M}}(2)$ satisfying

$$
\mathbb{X} \mathbb{Y}+\mathbb{Y} \mathbb{X}=a_{4} \mathbb{1}+b_{4} \mathbb{X}+d_{4} \mathbb{X}^{2} \text { and } \mathbb{Y}^{2}=\mathbb{1},
$$

where $a_{4}, b_{4}, d_{4} \in \mathbb{R}$. Multiplying the first relation in (4.11) with $\mathbb{X}$ on left (resp. right) we get

$$
\mathbb{X}^{2} \mathbb{Y}+\mathbb{X} \mathbb{Y}=a_{4} \mathbb{X}+b_{4} \mathbb{X}^{2}+d_{4} \mathbb{X}^{3}=\mathbb{X} \mathbb{Y} \mathbb{X}+\mathbb{Y}^{2}
$$

Hence, $\mathbb{X}^{2} \mathbb{Y}=\mathbb{Y}^{2}$. Multiplying the first relation in (4.11) with $\mathbb{Y}$ on right and using the second relation in (4.11), we get

$$
\mathbb{X}+\mathbb{Y} \mathbb{X}=a_{4} \mathbb{Y}+b_{4} \mathbb{X} \mathbb{Y}+d_{4} \mathbb{X}^{2} \mathbb{Y}
$$

Comparing the rows $\mathbb{X} \mathbb{Y}, \mathbb{Y} \mathbb{X}$ on both sides of (4.12) gives $b_{4}=0$. We now separate two possibilities depending on $d_{4}$.

Case 2.1.1: $d_{4}=0$ in (4.11). The relations (4.11) are

$$
\mathbb{X} \mathbb{Y}+\mathbb{Y} \mathbb{X}=a_{4} \mathbb{1}, \quad \mathbb{Y}^{2}=\mathbb{1}
$$

Using the second relation we can rewrite the first relation in the form

$$
\left(\mathbb{X}-\frac{a_{4}}{2} \mathbb{Y}\right) \mathbb{Y}+\mathbb{Y}\left(\mathbb{X}-\frac{a_{4}}{2} \mathbb{Y}\right)=\mathbf{0} .
$$

Applying an affine linear transformation $\phi_{6}(\mathbb{X}, \mathbb{Y})=\left(x-\frac{a_{4}}{2} y, y\right)$ to $\breve{\beta}$ we get $\widehat{\beta}$ with $\widehat{\mathcal{M}}(2)$ satisfying

$$
\mathbb{X} \mathbb{Y}+\mathbb{Y}=\mathbf{0}, \quad \mathbb{Y}^{2}=\mathbb{1} .
$$

Hence we are in the basic case 2 of Proposition 4.1 (1).

Case 2.1.2: $d_{4} \neq 0$ in (4.11). The relations (4.11) are

$$
\mathbb{X}^{2}-\frac{1}{d_{4}}(\mathbb{X} \mathbb{Y}+\mathbb{Y} \mathbb{X})=-\frac{a_{4}}{d_{4}} \mathbb{1} \quad \text { and } \quad \mathbb{Y}^{2}=\mathbb{1} .
$$

Summing together the first relation and the second relation multiplied by $\frac{1}{d_{4}^{2}}$ we get

$$
\frac{1}{d_{4}^{2}} \mathbb{Y}^{2}-\frac{1}{d_{4}}(\mathbb{X} \mathbb{Y}+\mathbb{Y} \mathbb{X})+\mathbb{X}^{2}=\left(\frac{1}{d_{4}^{2}}-\frac{a_{4}}{d_{4}}\right) \mathbb{1}
$$

Now we rewrite (4.13) in the form

$$
\left(\frac{1}{d_{4}} \mathbb{Y}-\mathbb{X}\right)^{2}=\left(\frac{1}{d_{4}^{2}}-\frac{a_{4}}{d_{4}}\right) \mathbb{1}
$$

Applying an affine linear transformation $\phi_{7}(X, Y)=\left(\frac{1}{d_{4}} y-X, Y\right)$ to $\breve{\beta}$ we get $\dot{\beta}$ with $\dot{\mathcal{M}}(2)$ satisfying

$$
\mathbb{X}^{2}=\left(\frac{1}{d_{4}^{2}}-\frac{a_{4}}{d_{4}}\right) \mathbb{1} \quad \text { and } \quad \mathbb{Y}^{2}=\mathbb{1}
$$

Hence we are in Case 1.

Case 2.2: $\mathbb{Y}^{2}=\mathbb{1}-\mathbb{X}^{2}$ in (4.9). Multiplying the relation (4.10) from the left by $\mathbb{X}$ (resp. $\mathbb{Y}$ ) and comparing the rows $\mathbb{X} \mathbb{Y}, \mathbb{Y} \mathbb{X}$ on both sides using Lemma 4.5 we conclude that $c_{3}=0$ (resp. $b_{3}=0$ ). Thus the relation of $\overline{\mathcal{M}}(2)$ are

$$
\mathbb{X} \mathbb{Y}+\mathbb{Y} \mathbb{X}=a_{3} \mathbb{1}+d_{3} \mathbb{X}^{2} \text { and } \mathbb{Y}^{2}+\mathbb{X}^{2}=\mathbb{1}
$$

Summing together the first relation and the second relation multiplied by $\alpha$ we get

$$
\alpha \mathbb{Y}^{2}+(\mathbb{X} \mathbb{Y}+\mathbb{Y} \mathbb{X})+\left(\alpha-d_{3}\right) \mathbb{X}^{2}=\left(\alpha+a_{3}\right) \mathbb{1}
$$


Choosing

we see that

$$
\alpha=\frac{1}{2} \sqrt{4+d_{3}^{2}}+\frac{d_{3}}{2}
$$

and thus (4.14) can be rewritten in the form

$$
\alpha>0, \quad \alpha-d_{3}>0 \quad \text { and } \quad \sqrt{\left(\alpha-d_{3}\right) \alpha}=1,
$$

$$
\left(\sqrt{\alpha-d_{3}} \mathbb{X}+\sqrt{\alpha} \mathbb{Y}\right)^{2}=\left(\alpha+a_{3}\right) \mathbb{1} .
$$

Applying an affine linear transformation $\phi_{8}(X, Y)=\left(X, \sqrt{\alpha-d_{3}} X+\sqrt{\alpha} Y\right)$ to $\bar{\beta}$ we get $\widehat{\beta}$ with $\widehat{\mathcal{M}}(2)$ satisfying

$$
\mathbb{Y}^{2}=\left(\alpha+a_{3}\right) \mathbb{1} \text { and } \mathbb{X} \mathbb{Y}+\mathbb{Y} \mathbb{X}=a_{4} \mathbb{1}+d_{4} \mathbb{X}^{2},
$$

where $a_{4}, d_{4} \in \mathbb{R}$. Since $\widehat{\mathcal{M}}(2)$ is psd of rank $5, \alpha+a_{3}>0$ and after normalization the relations (4.15) become

$$
\mathbb{Y}^{2}=\mathbb{1} \quad \text { and } \quad \mathbb{X} \mathbb{Y}+\mathbb{Y} \mathbb{X}=a_{5} \mathbb{1}+d_{5} \mathbb{X}^{2},
$$

where $a_{5}, d_{5} \in \mathbb{R}$. Hence we are in Case 2.1.

Case 2.3: $\mathbb{Y}^{2}=\mathbb{X}^{2}$ in (4.9). As in the first paragraph of Case 2.2 we conclude that the relations of $\overline{\mathcal{M}}(2)$ are

$$
\mathbb{X} \mathbb{Y}+\mathbb{Y}=a_{3} \mathbb{1}+d_{3} \mathbb{X}^{2} \text { and } \mathbb{Y}^{2}=\mathbb{X}^{2}
$$

Applying an affine linear transformation $\phi_{9}(X, Y)=(X+Y, Y-X)$ to $\widetilde{\beta}$ we get $\bar{\beta}$ with $\overline{\mathcal{M}}(2)$ satisfying

$$
\left(2-d_{3}\right) \mathbb{X}^{2}-\left(2+d_{3}\right) \mathbb{Y}^{2}=4 a_{3} \mathbb{1} \quad \text { and } \quad \mathbb{X} \mathbb{Y}+\mathbb{Y} \mathbb{X}=\mathbf{0},
$$

If $d_{3}=2$, then after normalization we come into Case 2.1. If $d_{3}=-2$, then we come into Case 2.1 after we apply a transformation $(X, Y) \mapsto(Y, X)$ to change the roles of $\mathbb{X}$ and $\mathbb{Y}$ and normalize. Otherwise we apply an affine linear transformation

to $\widetilde{\beta}$ and get $\breve{\beta}$ with $\breve{\mathcal{M}}(2)$ satisfying

$$
\phi_{10}(X, Y)=\left(\sqrt{\left|2-d_{3}\right|} X, \sqrt{\left|2+d_{3}\right|} Y\right)
$$

and one of the following:

$$
\mathbb{X}^{2}+\mathbb{Y}^{2}=4 a_{3} \mathbb{1} \quad \text { or } \quad \mathbb{X}^{2}-\mathbb{Y}^{2}=4 a_{3} \mathbb{1} \quad \text { or } \quad-\mathbb{X}^{2}-\mathbb{Y}^{2}=4 a_{3} \mathbb{1}
$$

The first and the last cases are equivalent, since the third relation can be rewritten as $\mathbb{X}^{2}+\mathbb{Y}^{2}=-4 a_{3} \mathbb{1}$. Thus we separate two possibilities in (4.16).

Case 2.3.1: $\mathbb{X}^{2}+\mathbb{Y}^{2}=4 a_{3} \mathbb{1}$ in (4.16). It is easy to see that $a_{3}>0$ (by $\breve{\mathcal{M}}(2)$ being psd of rank 5 , since otherwise $\beta_{Y^{2}}=\beta_{X^{2} Y^{2}}=\beta_{Y^{4}}=0$ ). Thus after the normalization we are in the basic case 1 of Proposition 4.1.

Case 2.3.2: $\mathbb{X}^{2}-\mathbb{Y}^{2}=4 a_{3} \mathbb{1}$ in (4.16). We may assume that $a_{3} \leq 0$ (otherwise we change the roles of $\mathbb{X}$ and $\mathbb{Y}$ ). If $a_{3}<0$, then after normalization we come into the basic case 3 . Otherwise $a_{3}=0$ and we are in the basic case 4 .

Case 2.4: $\mathbb{Y}^{2}=\mathbb{1}+\mathbb{X}^{2}$ in (4.9). As in the first paragraph of Case 2.2 we conclude that the relations of $\overline{\mathcal{M}}(2)$ are

$$
\mathbb{X} \mathbb{Y}+\mathbb{Y} \mathbb{X}=a_{3} \mathbb{1}+d_{3} \mathbb{X}^{2} \text { and } \mathbb{Y}^{2}=\mathbb{1}+\mathbb{X}^{2},
$$

and after applying an affine linear transformation $\phi_{9}(X, Y)=(X+Y, Y-X)$ to $\bar{\beta}$ to get $\breve{\beta}$ with $\breve{\mathcal{M}}(2)$ satisfying

$$
\left(2-d_{3}\right) \mathbb{X}^{2}-\left(2+d_{3}\right) \mathbb{Y}^{2}=\left(4 a_{3}-2 d_{3}\right) \mathbb{1} \quad \text { and } \quad \mathbb{X} \mathbb{Y}+\mathbb{Y} \mathbb{X}=2 \cdot \mathbb{1}
$$

If $d_{3}=2$, then after normalization we come into Case 2.1. If $d_{3}=-2$ then we come into Case 2.1 after we apply a transformation $(X, Y) \mapsto(Y, X)$ to change the roles of $\mathbb{X}$ and $\mathbb{Y}$ and normalize. Otherwise we apply an affine linear transformation

to $\breve{\beta}$ and get $\dot{\beta}$ with $\mathcal{M}(2)$ satisfying

$$
\phi_{11}(X, Y)=\left(\sqrt{\left|2-d_{3}\right|} X, \sqrt{\left|2+d_{3}\right|} Y\right)
$$

and one of the following

$$
\mathbb{X} \mathbb{Y}+\mathbb{Y} \mathbb{X}=2 \sqrt{\mid\left(4-d_{3}^{2} \mid\right.} \mathbb{1}
$$

$$
\mathbb{X}^{2}+\mathbb{Y}^{2}=\tilde{a} \mathbb{1} \quad \text { or } \quad \mathbb{X}^{2}-\mathbb{Y}^{2}=\tilde{a} \mathbb{1} \quad \text { or } \quad-\mathbb{X}^{2}-\mathbb{Y}^{2}=\tilde{a} \mathbb{1},
$$


where $\tilde{a}=4 a_{3}-2 d_{3}$. The first and the last cases are equivalent, since the third relation can be rewritten as $\mathbb{X}^{2}+\mathbb{Y}^{2}=-\tilde{a} \mathbb{1}$. Thus we separate two possibilities in (4.17).

Case 2.4.1: $\mathbb{X}^{2}+\mathbb{Y}^{2}=\tilde{a} \mathbb{1}$. It is easy to see that $\tilde{a}>0$ (by $\mathcal{M}(2)$ being psd of rank 5 , since otherwise $\beta_{Y^{2}}=\beta_{X^{2} Y^{2}}=$ $\left.\beta_{Y^{4}}=0\right)$. Hence after normalization we come into Case 2.2.

Case 2.4.2: $\mathbb{Y}^{2}-\mathbb{X}^{2}=\tilde{a} \mathbb{1}$. We may assume that $\tilde{a} \geq 0$ (otherwise we change the roles of $\mathbb{X}$ and $\mathbb{Y}$ ). If $\tilde{a}=0$, we are in Case 2.3. Otherwise we apply a transformation

$$
\phi_{12}(X, Y)=\left(X, X-\frac{2 \sqrt{\mid\left(4-d_{3}^{2} \mid\right.}}{\tilde{a}} Y\right)
$$

to $\hat{\beta}$ and get $\widehat{\beta}$ with $\widehat{\mathcal{M}}(2)$ satisfying

$$
\mathbb{Y}^{2}+\left(1-\frac{4\left(4-d_{3}^{2}\right)^{2}}{\tilde{a}^{2}}\right) \mathbb{X}^{2}=\mathbf{0} \text { and } \mathbb{X} \mathbb{Y}+\mathbb{Y} \mathbb{X}=-\tilde{a} \mathbb{1}+\tilde{a} \mathbb{X}^{2}
$$

It is easy to see that $1-\frac{4\left(4-d_{d}^{2}\right)^{2}}{\tilde{a}^{2}}<0$ (by $\hat{\mathcal{M}}(2)$ being psd of rank 5 , since otherwise $\beta_{Y^{4}}=\beta_{X^{2} Y^{2}}=\beta_{Y^{2}}=\beta_{X^{2}}=0$ ) and after a further normalization of $\mathbb{X}$ the relations of the corresponding matrix $\widehat{\mathcal{M}}(2)$ become

$$
\mathbb{Y}^{2}-\mathbb{X}^{2}=\mathbf{0} \quad \text { and } \quad \mathbb{X} \mathbb{Y}+\mathbb{Y} \mathbb{X}=-\hat{a} \mathbb{1}-\hat{a} \mathbb{X}^{2}, \quad \text { for some } \hat{a} \in \mathbb{R} \text {. }
$$

Hence we come into Case 2.3.

Case 3: The set $\left\{\mathbb{1}, \mathbb{X}, \mathbb{Y}, \mathbb{Y}^{2}, \mathbb{Y} \mathbb{X}\right\}$ is the basis for $\mathcal{C}_{\mathcal{M}_{2}}$.

Applying an affine linear transformation $(X, Y) \mapsto(Y, X)$ we come into Case 2 .

Now we prove Proposition 4.1 (2).

Proof of Proposition 4.1 (2). By Lemma 4.4 we have to consider 2 different cases.

Case 1: The set $\left\{\mathbb{1}, \mathbb{X}, \mathbb{Y}, \mathbb{X}^{2}, \mathbb{X} \mathbb{Y}, \mathbb{Y}\right\}$ is the basis for $\mathcal{C}_{\mathcal{M}_{2}}$.

By assumption there are constants $a_{i}, i=1, \ldots, 6$, such that

$$
\mathbb{Y}^{2}=a_{1} \mathbb{1}+a_{2} \mathbb{X}+a_{3} \mathbb{Y}+a_{4} \mathbb{X}^{2}+a_{5} \mathbb{X} \mathbb{Y}+a_{6} \mathbb{Y} \mathbb{X}
$$

By Lemma 4.2 the statement of Proposition 4.1 follows.

Case 2: The set $\left\{\mathbb{1}, \mathbb{X}, \mathbb{Y}, \mathbb{X}^{2}, \mathbb{X} \mathbb{Y}, \mathbb{Y}^{2}\right\}$ is the basis for $\mathcal{C}_{\mathcal{M}_{2}}$.

By assumption there are constants $a_{i}, i=1, \ldots, 6$, such that

$$
\mathbb{Y} \mathbb{X}=a_{1} \mathbb{1}+a_{2} \mathbb{X}+a_{3} \mathbb{Y}+a_{4} \mathbb{X}^{2}+a_{5} \mathbb{X} \mathbb{Y}+a_{6} \mathbb{Y}^{2}
$$

By comparing the rows $\mathbb{X} \mathbb{Y}, \mathbb{Y} \mathbb{X}$ of the both sides of equation we conclude that $a_{5}=-1$. We separate two cases.

Case 2.1: $a_{4} \neq 0$ or $a_{6} \neq 0$. By symmetry we may assume that $a_{6} \neq 0$. We rewrite the relation (4.18) as

$$
\mathbb{Y}^{2}=-\frac{a_{1}}{a_{6}} \mathbb{1}-\frac{a_{2}}{a_{6}} \mathbb{X}-\frac{a_{3}}{a_{6}} \mathbb{Y}-\frac{a_{4}}{a_{6}} \mathbb{X}^{2}-\frac{a_{5}}{a_{6}} \mathbb{X} \mathbb{Y}+\frac{1}{a_{6}} \mathbb{Y} \mathbb{X} .
$$

By Lemma 4.2 the statement of Proposition 4.1 follows.

Case 2.2: $a_{4}=a_{6}=0$. We rewrite the relation (4.18) as

$$
(\mathbb{X}+\mathbb{Y}) \mathbb{Y}+\mathbb{Y}(\mathbb{X}+\mathbb{Y})-2 \mathbb{Y}^{2}=a_{1} \mathbb{1}+a_{2}(\mathbb{X}+\mathbb{Y})+\left(a_{3}-a_{2}\right) \mathbb{Y}
$$

Applying an affine linear transformation $\phi_{1}(X, Y)=(X+Y, Y)$ to $\beta$ we get $\widetilde{\beta}$ with $\widetilde{\mathcal{M}}(2)$ satisfying

$$
\mathbb{X} \mathbb{Y}+\mathbb{Y}-2 \mathbb{Y}^{2}=a_{1} \mathbb{1}+a_{2} \mathbb{X}+\left(a_{3}-a_{2}\right) \mathbb{Y}
$$

By Lemma 4.2 the statement of Proposition 4.1 (2) follows. 


\section{ATOMS IN THE MINIMAL MEASURE OF RANKS 5 AND 6}

In this section we show that every nc sequence $\beta \equiv \beta^{(4)}$ which admits a nc measure with $\mathcal{M}_{2}$ in one of the basic cases of rank 5 or one of the first three basic cases of rank 6 given by Proposition 4.1, admits a minimal measure with all the atoms of special form; see Proposition 5.1 below. This form will be crucial in the subsequent sections where we will analyze each basic case separately to show that the atoms of size 2 are sufficient.

Proposition 5.1. Suppose a nc sequence $\beta \equiv \beta^{(4)}$ has a moment matrix $\mathcal{M}_{2}$ satisfying one of the column relations

$$
\mathbb{X} \mathbb{Y}+\mathbb{Y} \mathbb{X}=\mathbf{0} \quad \text { or } \quad \mathbb{Y}^{2}=\mathbb{1}-\mathbb{X}^{2} \quad \text { or } \quad \mathbb{Y}^{2}=\mathbb{1}+\mathbb{X}^{2}
$$

If $\beta$ admits a nc measure, then the atoms are of the following two forms:

(1) $\left(x_{i}, y_{i}\right) \in \mathbb{R}^{2}$.

(2) $\left(X_{i}, Y_{i}\right) \in\left(\mathbb{S R}^{2 t_{i} \times 2 t_{i}}\right)^{2}$ for some $t_{i} \in \mathbb{N}$ such that

$$
X_{i}=\left(\begin{array}{cc}
\gamma_{i} I_{t_{i}} & B_{i} \\
B_{i}^{t} & -\gamma_{i} I_{t_{i}}
\end{array}\right) \quad \text { and } \quad Y_{i}=\left(\begin{array}{cc}
\mu_{i} I_{t_{i}} & \mathbf{0} \\
\mathbf{0} & -\mu_{i} I_{t_{i}}
\end{array}\right)
$$

where $\gamma_{i} \geq 0, \mu_{i}>0$ and $B_{i}$ are $t_{i} \times t_{i}$ matrices.

Proof. Suppose $\mu$ is any nc measure representing $\beta$. By Theorem 2.2 every atom $\left(X_{i}, Y_{i}\right)$ in $\mu$ satisfies the relation (5.1).

Claim 1: We may assume that $X_{i} Y_{i}+Y_{i} X_{i}$ and $Y_{i}$ are diagonal matrices.

Observe that $X_{i} Y_{i}+Y_{i} X_{i}$ is symmetric and commutes with $Y_{i}$. Therefore after a orthogonal transformation we may assume that $X_{i} Y_{i}+Y_{i} X_{i}$ and $Y_{i}$ are diagonal matrices.

Claim 2: We may assume that the atoms $\left(X_{i}, Y_{i}\right)$ of size greater than 1 are of the forms

$$
X_{i}=\left(\begin{array}{cc}
D_{i 1} & B_{i} \\
B_{i}^{t} & D_{i 2}
\end{array}\right) \quad \text { and } \quad Y_{i}=\left(\begin{array}{cc}
\mu_{i} I_{n_{i 1}} & \mathbf{0} \\
\mathbf{0} & -\mu_{i} I_{n_{i 2}}
\end{array}\right) \text {, }
$$

where $\mu_{i}>0, n_{i 1}, n_{i 2} \in \mathbb{N}, D_{i 1} \in \mathbb{R}^{n_{i 1} \times n_{i 1}}$ and $D_{i 2} \in \mathbb{R}^{n_{i 2} \times n_{i 2}}$ are diagonal matrices and $B_{i} \in \mathbb{R}^{n_{i 1} \times n_{i 2}}$.

By an appropriate permutation we may assume that $Y_{i}$ is of the form

$$
Y_{i}=\bigoplus_{j=1}^{\ell_{i}}\left(\begin{array}{cc}
\mu_{j}^{(i)} I_{n_{i j}} & \mathbf{0} \\
\mathbf{0} & -\mu_{j}^{(i)} I_{m_{i j}}
\end{array}\right) \bigoplus \mathbf{0}_{m \times m}
$$

where $\ell_{i}, n_{i j}, m_{i j}, m \in \mathbb{N} \cup\{0\}, \mu_{j}^{(i)}>0$ and $\mu_{j_{1}}^{(i)} \neq \mu_{j_{2}}^{(i)}$ for $j_{1} \neq j_{2}$. Let

$$
X_{i}=\left(X_{p r}^{(i)}\right)_{p r}
$$

be the corresponding block decomposition of $X_{i}$. Since $X_{i} Y_{i}+Y_{i} X_{i}$ is diagonal, it follows that

(1) for $1 \leq p, r \leq \ell_{i}$ and $p \neq r$ we have that

$$
\begin{aligned}
{\left[X_{i} Y_{i}+Y_{i} X_{i}\right]_{2 p-1,2 r-1}=\left(\mu_{p}^{(i)}+\mu_{r}^{(i)}\right) X_{2 p-1,2 r-1}^{(i)}=\mathbf{0} } & \Rightarrow \quad X_{2 p-1,2 r-1}^{(i)}=\mathbf{0} \\
{\left[X_{i} Y_{i}+Y_{i} X_{i}\right]_{2 p-1,2 r}=\left(\mu_{p}^{(i)}-\mu_{r}^{(i)}\right) X_{2 p-1,2 r}^{(i)}=\mathbf{0} } & \Rightarrow \quad X_{2 p-1,2 r}^{(i)}=\mathbf{0} \\
{\left[X_{i} Y_{i}+Y_{i} X_{i}\right]_{2 p, 2 r}=-\left(\mu_{p}^{(i)}+\mu_{r}^{(i)}\right) X_{2 p, 2 r}^{(i)}=\mathbf{0} } & \Rightarrow \quad X_{2 p, 2 r}^{(i)}=\mathbf{0} .
\end{aligned}
$$

(2) for $1 \leq p \leq \ell_{i}$ we have that

$$
\begin{aligned}
{\left[X_{i} Y_{i}+Y_{i} X_{i}\right]_{2 p-1,2 \ell_{i}+1}=\mu_{p}^{(i)} X_{2 p-1,2 \ell_{i}+1}^{(i)}=\mathbf{0} } & \Rightarrow \quad X_{2 p-1,2 \ell_{i}+1}^{(i)}=\mathbf{0} \\
{\left[X_{i} Y_{i}+Y_{i} X_{i}\right]_{2 p, 2 \ell_{i}+1}=-\mu_{p}^{(i)} X_{2 p, 2 \ell_{i}+1}^{(i)}=\mathbf{0} } & \Rightarrow \quad X_{2 p, 2 \ell_{i}+1}^{(i)}=\mathbf{0} \\
{\left[X_{i} Y_{i}+Y_{i} X_{i}\right]_{2 \ell_{i}+1,2 p-1}=\mu_{p}^{(i)} X_{2 \ell_{i}+1,2 p-1}^{(i)}=\mathbf{0} } & \Rightarrow \quad X_{2 \ell_{i}+1,2 p-1}^{(i)}=\mathbf{0} \\
{\left[X_{i} Y_{i}+Y_{i} X_{i}\right]_{2 \ell_{i}+1,2 p}=-\mu_{p}^{(i)} X_{2 \ell_{i}+1,2 p}^{(i)}=\mathbf{0} } & \Rightarrow \quad X_{2 \ell_{i}+1,2 p}^{(i)}=\mathbf{0} .
\end{aligned}
$$


(3) for $1 \leq p=r \leq \ell_{i}$ we have that

$$
\begin{aligned}
{\left[X_{i} Y_{i}+Y_{i} X_{i}\right]_{2 p-1,2 p-1}=2 \mu_{p}^{(i)} X_{2 p-1,2 p-1}^{(i)} \text { is diagonal } } & \Rightarrow X_{2 p-1,2 p-1}^{(i)} \text { is diagonal }, \\
{\left[X_{i} Y_{i}+Y_{i} X_{i}\right]_{2 p, 2 p}=-2 \mu_{p}^{(i)} X_{2 p, 2 p}^{(i)} \text { is diagonal } } & \Rightarrow \quad X_{2 p, 2 p}^{(i)} \text { is diagonal. }
\end{aligned}
$$

So $X_{i}$ is of the form

$$
X_{i}=\bigoplus_{j=1}^{\ell_{i}}\left(\begin{array}{cc}
X_{11}^{(i j)} & X_{12}^{(i j)} \\
\left(X_{12}^{(i j)}\right)^{t} & X_{22}^{(i j)}
\end{array}\right) \bigoplus X_{\ell_{i}+1}^{(i)} .
$$

Thus we can replace the atom $\left(X_{i}, Y_{i}\right)$ with the atoms of the form

$$
\tilde{X}_{i j}=\left(\begin{array}{cc}
X_{11}^{(i j)} & X_{12}^{(i j)} \\
\left(X_{12}^{(i j)}\right)^{t} & X_{22}^{(k i j)}
\end{array}\right) \quad \text { and } \quad \tilde{Y}_{i j}=\left(\begin{array}{cc}
\mu_{j}^{(i)} I_{n_{i j}} & \mathbf{0} \\
\mathbf{0} & -\mu_{j}^{(i)} I_{m_{i j}}
\end{array}\right)
$$

or

$$
\widetilde{X}_{i j}=X_{\ell_{i}+1}^{(i)} \quad \text { and } \quad \widetilde{Y}_{i j}=\mathbf{0} .
$$

By orthogonal transformation the atom (5.4) can be replaced by the atom

$$
\widehat{X}_{i j}=D_{\ell_{i}+1}^{(i)} \text { and } \quad \widetilde{Y}_{i j}=\mathbf{0},
$$

where $D_{\ell_{i}+1}^{(i)}$ is a diagonal matrix and further on by atoms of size 1 of the form $(x, 0)$, where $x$ runs over the diagonal of $D_{\ell_{i}+1}^{(i)}$. Hence we may assume that the atoms of size greater than 1 in the representing measure for $\beta$ are of the form (5.3). Further on, by appropriate orthogonal transformation we may assume that they are of the form (5.2). This proves the claim.

Claim 3: We may assume that the atoms $\left(X_{i}, Y_{i}\right)$ of size greater than 1 are of the forms

$$
X_{i}=\left(\begin{array}{cc}
\gamma_{i} I_{t_{i}} & B_{i} \\
B_{i}^{t} & -\gamma_{i} I_{t_{i}}
\end{array}\right) \quad \text { and } \quad Y_{i}=\left(\begin{array}{cc}
\mu_{i} I_{t_{i}} & \mathbf{0} \\
\mathbf{0} & -\mu_{i} I_{t_{i}}
\end{array}\right)
$$

where $\gamma_{i} \geq 0, \mu_{i}>0$ and $B_{i}$ are $t_{i} \times t_{i}$ matrices for some $t_{i} \in \mathbb{N}$.

First we prove Claim 3 in case we have $\mathbb{X} \mathbb{Y}+\mathbb{Y} \mathbb{X}=\mathbf{0}$ in (5.1). Let us prove that we may assume invertibility of $X_{i}$. After applying an orthogonal transformation to $\left(X_{i}, Y_{i}\right)$ we have $X_{i}=\left(\begin{array}{cc}\mathbf{0} & \mathbf{0} \\ \mathbf{0} & \widehat{X}_{i}\end{array}\right)$ where $\widehat{X}$ is invertible and $Y_{i}=\left(\begin{array}{cc}Y_{i 1} & Y_{i 2} \\ Y_{i 2}^{t} & Y_{i 3}\end{array}\right)$. From $X_{i} Y_{i}+Y_{i} X_{i}=\mathbf{0}$ it follows that $Y_{i 2} \widehat{X}_{i}=\mathbf{0}$. Since $\widehat{X}_{i}$ is invertible, $Y_{i 2}=\mathbf{0}$. Hence we can replace the atom $\left(X_{i}, Y_{i}\right)$ with the atoms $\left(\mathbf{0}, Y_{i 1}\right)$ and $\left(\widehat{X}_{i}, Y_{i 3}\right)$. Since the atom $\left(\mathbf{0}, Y_{i 1}\right)$ can be further replaced with the atoms of size 1 , we may assume the $X_{i}$ is invertible.

Observe that in (3) from the proof of Claim 2 we have

$$
\begin{aligned}
\mathbf{0}=\left[X_{i} Y_{i}+Y_{i} X_{i}\right]_{2 p-1,2 p-1}=2 \mu_{p}^{(i)} X_{2 p-1,2 p-1}^{(i)} & \Rightarrow \quad X_{2 p-1,2 p-1}^{(i)}=\mathbf{0}, \\
\mathbf{0}=\left[X_{i} Y_{i}+Y_{i} X_{i}\right]_{2 p, 2 p}=-2 \mu_{p}^{(i)} X_{2 p, 2 p}^{(i)} & \Rightarrow \quad X_{2 p, 2 p}^{(i)}=\mathbf{0} .
\end{aligned}
$$

Therefore $X_{i}$ in (5.2) is of the form $X_{i}=\left(\begin{array}{cc}\mathbf{0} & B_{i} \\ B_{i}^{t} & \mathbf{0}\end{array}\right)$ with $B_{i} \in \mathbb{R}^{n_{i 1} \times n_{i 2}}$ and $n_{i 1}=n_{i 2}$ by the invertibility of $X_{i}$. This proves Claim 3 in case we have $\mathbb{X} \mathbb{Y}+\mathbb{Y} \mathbb{X}=\mathbf{0}$ in (5.1).

It remains to prove Claim 3 in case we have $\mathbb{Y}^{2}=\mathbb{1} \pm \mathbb{X}^{2}$ in (5.1). By Claim 2 and after an appropriate permutation we may assume that $X_{i}, Y_{i}$ are of the form (5.2) with

$$
D_{i 1}=\bigoplus_{j=1}^{p_{i}} \lambda_{j}^{(i)} I_{s_{i j}} \quad \text { and } \quad D_{i 2}=\bigoplus_{j=1}^{r_{i}} \gamma_{j}^{(i)} I_{v_{i j}}
$$

where $p_{i}, s_{i j}, r_{i}, v_{i j} \in \mathbb{N}$ and

$$
\lambda_{1}^{(i)}>\lambda_{2}^{(i)}>\ldots>\lambda_{p_{i}}^{(i)} \quad \text { and } \quad \gamma_{1}^{(i)}>\gamma_{2}^{(i)}>\ldots>\gamma_{r_{i}}^{(i)}
$$


Let

$$
B_{i}=\left(B_{p r}^{(i)}\right)_{p r}
$$

be the corresponding block decomposition of $B_{i}$, where

$$
B_{p r}^{(i)} \in \mathbb{R}^{s_{i p} \times v_{i r}}
$$

for $p=1, \ldots, p_{i}, r=1, \ldots, r_{i}$. Calculating $X_{i}^{2}$ we get that

$$
X_{i}^{2}=\left(\begin{array}{cc}
D_{i 1}^{2}+B_{i} B_{i}^{t} & D_{i 1} B_{i}+B_{i}^{t} D_{i 2} \\
B_{i}^{t} D_{i 1}+D_{i 2} B_{i} & B_{i}^{t} B_{i}+D_{i 2}^{2}
\end{array}\right) .
$$

Since $X_{i}^{2}$ is a diagonal matrix, we conclude that

$$
D_{i 1} B_{i}+B_{i}^{t} D_{i 2}=\mathbf{0} .
$$

Thus

$$
\left[D_{i 1} B_{i}+B_{i}^{t} D_{i 2}\right]_{p r}=\left(\lambda_{p}^{(i)}+\gamma_{r}^{(i)}\right) B_{p r}^{(i)}=\mathbf{0},
$$

for $1 \leq p \leq p_{i}, 1 \leq r \leq r_{i}$. We conclude that

$$
\lambda_{p}^{(i)}=-\gamma_{r}^{(i)} \quad \text { or } \quad B_{p r}^{(i)}=\mathbf{0} .
$$

So in every row and every column in the block decomposition of $B_{i}$ at most one block $B_{p r}^{(i)}$ is possibly nonzero, i.e., $B_{p r}^{(i)}$ may be nonzero if and only if $\lambda_{p}^{(i)}=-\gamma_{r}^{(i)}$ So after a suitable permutation $X_{i}$ has the following block decomposition

$$
\begin{aligned}
& X_{i}=\bigoplus_{\substack{1 \leq p \leq p_{i} \\
1 \leq r \leq r_{i} \\
\lambda_{p}^{(i)}+\gamma_{r}^{(i)}=0}}\left(\begin{array}{cc}
\lambda_{p}^{(i)} I_{s_{i p}} & B_{p r}^{(i)} \\
\left(B_{p r}^{(i)}\right)^{t} & \gamma_{r}^{(i)} I_{v_{i r}}
\end{array}\right) \bigoplus \bigoplus_{\substack{1 \leq p \leq p_{k} \\
\lambda_{p}^{(i)} \neq-\gamma_{r}^{(i)} \\
\lambda_{p}}}\left(\lambda_{p}^{(i)} I_{s_{i p}}\right) \\
& \bigoplus \bigoplus_{\substack{1 \leq r \leq r_{k} \\
\lambda_{p}^{(i)} \neq-\gamma_{r}^{(i)} \forall p}}\left(\gamma_{r}^{(i)} I_{v_{i r}}\right)
\end{aligned}
$$

The corresponding block decomposition of $Y_{i}$ is of the form

$$
\begin{aligned}
& Y_{i}= \bigoplus_{\substack{1 \leq p \leq p_{i} \\
1 \leq r \leq r_{i} \\
\lambda_{p}^{(i)}+\gamma_{r}^{(i)}=0}}\left(\begin{array}{cc}
\mu_{i} I_{s_{i p}} & 0 \\
0 & -\mu_{i} I_{v_{i r}}
\end{array}\right) \bigoplus \bigoplus_{\substack{1 \leq p \leq p_{k} \\
\lambda_{p}^{(i)} \neq-\gamma_{r}^{(i)} \forall r}}\left(\mu_{i} I_{s_{i p}}\right) \\
& \bigoplus \bigoplus_{\substack{1 \leq r \leq r_{k} \\
\lambda_{p}^{(i)} \neq-\gamma_{r}^{(i)} \forall p}}\left(-\mu_{i} I_{v_{i r}}\right) .
\end{aligned}
$$

Thus we can replace the atom $\left(X_{i}, Y_{i}\right)$ with the atoms of the form

$$
\widetilde{X}_{i j}=\left(\begin{array}{cc}
\lambda_{p}^{(i)} I_{s_{i p}} & B_{p r}^{(i)} \\
\left(B_{p r}^{(i)}\right)^{t} & -\lambda_{p}^{(i)} I_{v_{i r}}
\end{array}\right) \quad \text { and } \quad \widetilde{Y}_{i j}=\left(\begin{array}{cc}
\mu_{i} I_{s_{i p}} & 0 \\
0 & -\mu_{i} I_{v_{i r}}
\end{array}\right)
$$

or

$$
\widetilde{X}_{i j}=\lambda_{p}^{(i)} \quad \text { and } \quad \widetilde{Y}_{i j}=\mu_{i}
$$

or

$$
\widetilde{X}_{i j}=\gamma_{r}^{(i)} \text { and } \quad \tilde{Y}_{i j}=-\mu_{i}
$$

Hence we may assume that the atoms $\left(X_{i}, Y_{i}\right)$ of size greater than 1 in the representing measure for $\mathcal{M}_{2}$ are of the form (5.7). Now

$$
X_{i}^{2}=\left(\begin{array}{cc}
\left(\lambda_{p}^{(i)}\right)^{2} I_{s_{i p}}+B_{p r}^{(i)}\left(B_{p r}^{(i)}\right)^{t} & \mathbf{0} \\
\mathbf{0} & \left(B_{p r}^{(i)}\right)^{t} B_{p r}^{(i)}+\left(\lambda_{p}^{(i)}\right)^{2} I_{v_{i r}}
\end{array}\right)
$$

Since

$$
X_{i}^{2}=\mathbb{1} \pm Y_{i}^{2}=\left(\begin{array}{cc}
\left(1 \pm \mu_{i}^{2}\right) I_{s_{i p}} & \mathbf{0} \\
\mathbf{0} & \left(1 \pm \mu_{i}^{2}\right) I_{v_{i r}}
\end{array}\right)
$$


it follows that

$$
\begin{aligned}
& B_{p r}^{(i)}\left(B_{p r}^{(i)}\right)^{t}=\left(1 \pm \mu_{i}^{2}-\left(\lambda_{p}^{(i)}\right)^{2}\right) I_{s_{i p}} \\
& \left(B_{p r}^{(i)}\right)^{t} B_{p r}^{(i)}=\left(1 \pm \mu_{i}^{2}-\left(\lambda_{p}^{(i)}\right)^{2}\right) I_{v_{i r}} .
\end{aligned}
$$

We separate two cases according to the value of $1 \pm \mu_{i}^{2}-\left(\lambda_{p}^{(i)}\right)$.

Case 1: $1 \pm \mu_{i}^{2}-\left(\lambda_{p}^{(i)}\right)=0$.

It follows that $B_{p r}^{(i)}=\mathbf{0}$. Then $X_{i}$ is diagonal and commutes with $Y_{i}$. Therefore the atom $\left(X_{i}, Y_{i}\right)$ can be replaced by the atoms $\left(\lambda_{p}^{(i)}, \mu_{i}\right)$ and $\left(-\lambda_{p}^{(i)},-\mu_{i}\right)$.

Case 2: $1 \pm \mu_{i}^{2}-\left(\lambda_{p}^{(i)}\right) \neq 0$.

From (5.8) and (5.9) it follows that

$$
\begin{aligned}
& s_{i p}=\operatorname{rank}\left(B_{p r}^{(i)}\left(B_{p r}^{(i)}\right)^{t}\right) \leq \min \left(\operatorname{rank}\left(B_{p r}^{(i)}\right), \operatorname{rank}\left(\left(B_{p r}^{(i)}\right)^{t}\right)\right) \leq \min \left(s_{i p}, v_{i r}\right) \\
& v_{i r}=\operatorname{rank}\left(\left(B_{p r}^{(i)}\right)^{t} B_{p r}^{(i)}\right) \leq \min \left(\operatorname{rank}\left(\left(B_{p r}^{(i)}\right)^{t}\right), \operatorname{rank}\left(B_{p r}^{(i)}\right)\right) \leq \min \left(v_{i r}, s_{i p}\right) .
\end{aligned}
$$

It follows from (5.10) and (5.11) that $s_{i p}=v_{i r}$ in (5.7) which proves Claim 3 and concludes the proof of Proposition 5.1 .

\section{Solution OF THE BQTMP FOR $\mathcal{M}_{2}$ OF RANK 5}

In this section we solve the BQTMP for $\mathcal{M}_{2}$ of rank 5. By Proposition 4.1 it suffices to solve four basic cases. In Subsections $6.1,6.2,6.3,6.4$ we study these cases separately. We characterize exactly when $\mathcal{M}_{2}$ admits a nc measure, see Theorems 6.5, 6.8, 6.11 and 6.14. Moreover, we characterize type and uniqueness of the minimal measures. In particular, the minimal measure is almost always unique (up to orthogonal equivalence), except in one subcase for which there are two minimal measures, and there is always exactly one atom from $\left(\mathbb{S R}^{2 \times 2}\right)^{2}$ in the minimal measure and up to three atoms from $\mathbb{R}^{2}$.

Let $(X, Y) \in\left(\mathbb{S R}^{t \times t}\right)^{2}$ where $t \in \mathbb{N}$. We denote by $\mathcal{M}_{2}^{(X, Y)}$ the moment matrix generated by $(X, Y)$, i.e., $\beta_{w(X, Y)}=\operatorname{Tr}(w(X, Y))$ for every $|w(X, Y)| \leq 4$.

The following proposition will be used in all four basic cases to prove that if $\beta$ admits a nc measure, then it has a representing measure with the atoms of size at most 2.

Proposition 6.1. Let us fix a pair $\left(R_{1}, R_{2}\right)$ of the basic case relations given by Proposition 4.1 (1). If every sequence $\beta \equiv \beta^{(4)}$ with $\beta_{X}=\beta_{Y}=\beta_{X^{3}}=0$ and a moment matrix $\mathcal{M}_{2}(\beta)$ of rank 5 with column relations $R_{1}$ and $R_{2}$, admits a nc measure with atoms of size at most 2 , then every nc sequence $\widetilde{\beta} \equiv \widetilde{\beta}^{(4)}$ which admits a nc measure and has a moment matrix $\widetilde{\mathcal{M}}_{2}$ of rank 5 with column relations $R_{1}$ and $R_{2}$, admits a nc measure with atoms of size at most 2 .

Proof. Suppose $\widetilde{\beta}$ admit a nc measure and has a moment matrix $\widetilde{\mathcal{M}}_{2}$ of rank 5 with column relations $R_{1}$ and $R_{2}$. By Proposition 5.1 we may assume that all the atoms $\left(X_{i}, Y_{i}\right) \in\left(\mathbb{S R}^{u_{i} \times u_{i}}\right)^{2}$ of size $u_{i}>1$ are of the form

$$
X_{i}=\left(\begin{array}{cc}
\gamma_{i} I_{t_{i}} & B_{i} \\
B_{i}^{t} & -\gamma_{i} I_{t_{i}}
\end{array}\right), \quad Y_{i}=\left(\begin{array}{cc}
\mu_{i} I_{t_{i}} & \mathbf{0} \\
\mathbf{0} & -\mu_{i} I_{t_{i}}
\end{array}\right),
$$

where $\gamma_{i} \geq 0, \mu_{i}>0$ and $B_{i}$ are $t_{i} \times t_{i}$ matrices. Calculating $X_{i}^{3}$ we get

$$
X_{i}^{3}=\left(\begin{array}{cc}
\gamma_{i}\left(\gamma_{i}^{2} I_{t_{i}}+B_{i} B_{i}^{t}\right) & \left(\gamma_{i}^{2} I_{t_{i}}+B_{i} B_{i}^{t}\right) B_{i} \\
\left(\gamma_{i}^{2} I_{t_{i}}+B_{i}^{t} B_{i}\right) B_{i}^{t} & -\gamma_{i}\left(\gamma_{i}^{2} I_{t_{i}}+B_{i}^{t} B_{i}\right)
\end{array}\right)
$$

Therefore $\mathcal{M}_{2}^{\left(X_{i}, Y_{i}\right)}$ satisfies $\beta_{X}=\beta_{Y}=\beta_{X^{3}}=0$. By assumption the atom $\left(X_{i}, Y_{i}\right)$ can be replaced by the atoms of size at most 2.

6.1. Pair $\mathbb{X} \mathbb{Y}+\mathbb{Y} \mathbb{X}=\mathbf{0}$ and $\mathbb{Y}^{2}+\mathbb{X}^{2}=\mathbb{1}$. In this subsection we study a nc sequence $\beta \equiv \beta^{(4)}$ with a moment matrix $\mathcal{M}_{2}$ of rank 5 satisfying the relations $\mathbb{X} \mathbb{Y}+\mathbb{Y} \mathbb{X}=\mathbf{0}$ and $\mathbb{Y}^{2}+\mathbb{X}^{2}=\mathbb{1}$. In Theorem 6.5 we characterize exactly when $\beta$ admits a measure. Moreover, we classify type and uniqueness of the minimal measure.

The form of $\mathcal{M}_{2}$ is given by the following proposition. 
Proposition 6.2. Suppose $\beta \equiv \beta^{(4)}$ is a nc sequence with a moment matrix $\mathcal{M}_{2}$ of rank 5 satisfying the relations

$$
\mathbb{X} \mathbb{Y}+\mathbb{Y}=\mathbf{0} \text { and } \mathbb{X}^{2}+\mathbb{Y}^{2}=\mathbb{1}
$$

Then $\mathcal{M}_{2}$ is of the form

$$
\left(\begin{array}{ccccccc}
\beta_{1} & \beta_{X} & \beta_{Y} & \beta_{X^{2}} & 0 & 0 & \beta_{1}-\beta_{X^{2}} \\
\beta_{X} & \beta_{X^{2}} & 0 & \beta_{X} & 0 & 0 & 0 \\
\beta_{Y} & 0 & \beta_{1}-\beta_{X^{2}} & 0 & 0 & 0 & \beta_{Y} \\
\beta_{X^{2}} & \beta_{X} & 0 & \beta_{X^{4}} & 0 & 0 & \beta_{X^{2}}-\beta_{X^{4}} \\
0 & 0 & 0 & 0 & \beta_{X^{2}}-\beta_{X^{4}} & -\beta_{X^{2}}+\beta_{X^{4}} & 0 \\
0 & 0 & 0 & 0 & -\beta_{X^{2}}+\beta_{X^{4}} & \beta_{X^{2}}-\beta_{X^{4}} & 0 \\
\beta_{1}-\beta_{X^{2}} & 0 & \beta_{Y} & \beta_{X^{2}}-\beta_{X^{4}} & 0 & 0 & \beta_{1}-2 \beta_{X^{2}}+\beta_{X^{4}}
\end{array}\right) .
$$

Proof. The relations (6.1) give us the following system in $\mathcal{M}_{2}$

$$
\begin{array}{rrr}
2 \beta_{X Y}=0, & \beta_{X^{2} Y^{2}}+\beta_{X Y X Y}=0, & \beta_{Y^{3}}=\beta_{Y}-\beta_{X^{2} Y}, \\
2 \beta_{X^{2} Y}=0, & 2 \beta_{X Y^{3}}=0, & \beta_{X^{2} Y^{2}}=\beta_{X^{2}}-\beta_{X^{4}}, \\
2 \beta_{X Y^{2}}=0, & \beta_{Y^{2}}=\beta_{1}-\beta_{X^{2}}, & \beta_{X Y^{3}}=\beta_{X Y}-\beta_{X^{3} Y}, \\
2 \beta_{X^{3} Y}=0, & \beta_{X Y^{2}}=\beta_{X}-\beta_{X^{3}}, & \beta_{Y^{4}}=\beta_{Y^{2}}-\beta_{X^{2} Y^{2}} .
\end{array}
$$

The solution to (6.3) is given by

$$
\begin{aligned}
& \beta_{X}=\beta_{X^{3}}, \\
& \beta_{X Y X Y}=\beta_{X^{4}}-\beta_{X^{2}}, \\
& \beta_{X Y}=\beta_{X^{2} Y}=\beta_{X Y^{2}}=\beta_{X^{3} Y}=\beta_{X Y^{3}}=0 \text {, } \\
& \beta_{Y^{4}}=\beta_{1}-2 \beta_{X^{2}}+\beta_{X^{4}}, \\
& \beta_{Y^{3}}=\beta_{Y}, \\
& \beta_{X^{2} Y^{2}}=\beta_{X^{2}}-\beta_{X^{4}},
\end{aligned}
$$

and thus $\mathcal{M}_{2}$ takes the form (6.2).

Proposition 6.3. Suppose $\beta \equiv \beta^{(4)}$ is a normalized nc sequence with a moment matrix $\mathcal{M}_{2}$ of rank 5 satisfying the relations $\mathbb{X} \mathbb{Y}+\mathbb{Y} \mathbb{X}=\mathbf{0}$ and $\mathbb{X}^{2}+\mathbb{Y}^{2}=\mathbb{1}$. Then $\mathcal{M}_{2}$ is positive semidefinite if and only if

$$
\left|\beta_{X}\right|<\beta_{X^{2}}<1, \quad\left|\beta_{Y}\right|<\left(1-\beta_{X^{2}}\right), \quad c<\beta_{X^{4}}<\beta_{X^{2}},
$$

where

$$
c:=\frac{-\beta_{X^{2}}^{3}+\beta_{X^{2}}^{4}-\beta_{X}^{2}+\beta_{Y}^{2} \beta_{X}^{2}+3 \beta_{X^{2}} \beta_{X}^{2}-2 \beta_{X^{2}}^{2} \beta_{X}^{2}}{-\beta_{X^{2}}+\beta_{Y}^{2} \beta_{X^{2}}+\beta_{X^{2}}^{2}+\beta_{X}^{2}-\beta_{X^{2}} \beta_{X}^{2}} .
$$

In the proof of Proposition 6.3 we will need the following lemma.

Lemma 6.4. Let $A \in \mathbb{S R}^{t \times t}$ be a real symmetric $t \times t$ matrix and $A^{\prime} \in \mathbb{S R}^{(t+u) \times(t+u)}$ a real symmetric $(t+u) \times(t+u)$ matrix of the form

$$
A^{\prime}=\left(\begin{array}{cc}
A & B \\
B^{t} & C
\end{array}\right)
$$

where $B \in \mathbb{R}^{t \times u}, C \in \mathbb{S R}^{u \times u}$. Suppose that $\operatorname{rank}(A)=\operatorname{rank}\left(A^{\prime}\right)$. Then $A$ is positive semidefinite if and only if $A^{\prime}$ is positive semidefinite.

Proof. Since $A$ is a principal submatrix of $A^{\prime}, A^{\prime}$ being psd implies that $A$ is psd. This proves the implication $(\Leftarrow)$. It remains to prove the implication $(\Rightarrow)$. The assumption $\operatorname{rank}(A)=\operatorname{rank}\left(A^{\prime}\right)$ implies that there is a matrix $W \in \mathbb{R}^{t \times u}$ such that $B=A W$ and $C=B^{t} W=W^{t} A W$. By a theorem of Smul'jan [Smu59], $A$ being psd implies that $A^{\prime}$ is psd.

Proof of Proposition 6.3. Write $\mathcal{M}_{2}$ as $\mathcal{M}_{2}=\left(\begin{array}{cc}A & B \\ B^{t} & C\end{array}\right)$ where

$$
A=\left.\mathcal{M}_{2}\right|_{\left\{\mathbb{1}, \mathbb{X}, \mathbb{Y}, \mathbb{X}^{2}, \mathbb{X} \mathbb{Y}\right\}}, \quad B=\left.\mathcal{M}_{2}\right|_{\left\{\mathbb{1}, \mathbb{X}, \mathbb{Y}, \mathbb{X}^{2}, \mathbb{X} \mathbb{Y}\right\},\left\{\mathbb{Y X}, \mathbb{Y}^{2}\right\}}, \quad C=\left.\mathcal{M}_{2}\right|_{\left\{\mathbb{Y X}, \mathbb{Y}^{2}\right\}} .
$$

Since $\mathcal{M}_{2}$ satisfies the relations $\mathbb{X} \mathbb{Y}+\mathbb{Y} \mathbb{X}=0$ and $\mathbb{X}^{2}+\mathbb{Y}^{2}=\mathbb{1}$, it follows that $\operatorname{rank}\left(\mathcal{M}_{2}\right)=\operatorname{rank}(A)=5$. Now by Lemma $6.4, \mathcal{M}_{2}$ is psd if and only if $A$ is psd. Since $\operatorname{rank}(A)=5, A$ is psd if and only if $A$ is positive definite (pd). By Sylvester's criterion $A$ is pd if and only if all its principal minors are positive.

First we will prove that the conditions in (6.4) are necessary for $A$ being pd. Since

$$
\left.A\right|_{\{\mathbb{X}\}}=\beta_{X^{2}}>0,\left.\quad A\right|_{\{\mathbb{Y}\}}=1-\beta_{X^{2}}>0,\left.\quad A\right|_{\{\mathbb{X} \mathbb{Y}\}}=\beta_{X^{2}}-\beta_{X^{4}}>0,
$$


we have that $1>\beta_{X^{2}}>\beta_{X^{4}}>0$. Now

$$
0<\operatorname{det}\left(\left.A\right|_{\left\{\mathbb{X}, \mathbb{X}^{2}\right\}}+\left(\begin{array}{cc}
0 & 0 \\
0 & \beta_{X^{2}}-\beta_{X^{4}}
\end{array}\right)\right)=\beta_{X^{2}}^{2}-\beta_{X}^{2},
$$

implies that $\beta_{X^{2}}>\left|\beta_{X}\right|$. Further on,

$$
0<\operatorname{det}\left(\left.A\right|_{\left\{1, \mathbb{X}, \mathbb{Y}, \mathbb{X}^{2}\right\}}+\left(\begin{array}{cccc}
0 & 0 & 0 & 0 \\
0 & 0 & 0 & 0 \\
0 & 0 & 0 & 0 \\
0 & 0 & 0 & \beta_{X^{2}}-\beta_{X^{4}}
\end{array}\right)\right)=\left(\beta_{X^{2}}^{2}-\beta_{X}^{2}\right)\left(\left(1-\beta_{X^{2}}\right)^{2}-\beta_{Y}^{2}\right),
$$

gives $1-\beta_{X^{2}}>\left|\beta_{Y}\right|$. Finally, $\operatorname{det}\left(\left.A\right|_{\left\{\mathbb{1}, \mathbb{X}, \mathbb{Y}, \mathbb{X}^{2}\right\}}\right)>0$ implies that $\beta_{X^{4}}>c$.

It remains to prove that the conditions in (6.4) are sufficient for $A$ being pd. The principal minors $\alpha_{i}, i=1, \ldots, 5$, of $A$ are

$$
\begin{aligned}
& \alpha_{1}=1, \quad \alpha_{2}=\beta_{X^{2}}-\beta_{X}^{2}, \quad \alpha_{3}=\left(\beta_{X^{2}}-\beta_{X}^{2}\right)\left(1-\beta_{X^{2}}\right)-\beta_{X^{2}} \beta_{Y}^{2}, \\
& \alpha_{4}=d+\alpha_{3} \beta_{X^{4}}, \quad \alpha_{5}=\alpha_{4}\left(\beta_{X^{2}}-\beta_{X^{4}}\right),
\end{aligned}
$$

where

$$
d=-\beta_{X^{2}}^{3}+\beta_{X^{2}}^{4}-\beta_{X}^{2}+\beta_{Y}^{2} \beta_{X}^{2}+3 \beta_{X^{2}} \beta_{X}^{2}-2 \beta_{X^{2}}^{2} \beta_{X}^{2},
$$

Now, if (6.4) is true, then clearly $\alpha_{1}, \alpha_{2}>0$ and $\alpha_{5}>0$ if $\alpha_{4}>0$. It remains to prove that $\alpha_{3}>0$ and $\alpha_{4}>0$. Now $\alpha_{3}>0$ if and only if

$$
\beta_{Y}^{2}<\frac{\left(\beta_{X^{2}}-\beta_{X}^{2}\right)\left(1-\beta_{X^{2}}\right)}{\beta_{X^{2}}}=\left(1-\frac{\beta_{X}^{2}}{\beta_{X^{2}}}\right)\left(1-\beta_{X^{2}}\right) .
$$

Since $\left(1-\beta_{X^{2}}\right)^{2}<\left(1-\frac{\beta_{X}^{2}}{\beta_{X^{2}}}\right)\left(1-\beta_{X^{2}}\right)$, the inequality $\left|\beta_{Y}\right|<1-\beta_{X^{2}}$ implies that (6.6) is true. Finally, note that $\alpha_{4}>0$ if and only if $\beta_{X^{4}}>c$.

The following theorem characterizes normalized nc sequences $\beta$ with a moment matrix $\mathcal{M}_{2}$ of rank 5 satisfying the relations $\mathbb{X} \mathbb{Y}+\mathbb{Y} \mathbb{X}=\mathbf{0}$ and $\mathbb{X}^{2}+\mathbb{Y}^{2}=\mathbb{1}$, which admit a nc measure.

Theorem 6.5. Suppose $\beta \equiv \beta^{(4)}$ is a normalized nc sequence with a moment matrix $\mathcal{M}_{2}$ of rank 5 satisfying the relations $\mathbb{X} \mathbb{Y}+\mathbb{Y} \mathbb{X}=\mathbf{0}$ and $\mathbb{X}^{2}+\mathbb{Y}^{2}=\mathbb{1}$. Then $\beta$ admits a nc measure if and only if

$$
\left|\beta_{Y}\right|<1-\left|\beta_{X}\right|,\left|\beta_{X}\right|<\beta_{X^{2}}<1-\left|\beta_{Y}\right|, c \leq \beta_{X^{4}}<\beta_{X^{2}},
$$

where

$$
c=\frac{-\beta_{X^{2}}^{2}-\left|\beta_{X}\right|+2 \beta_{X^{2}}\left|\beta_{X}\right|+\left|\beta_{Y} \beta_{X}\right|}{-1+\left|\beta_{Y}\right|+\left|\beta_{X}\right|} .
$$

Moreover, the minimal measure is unique (up to orthogonal equivalence) and of type:

- $(1,1)$ if and only if $\beta_{X} \beta_{Y}=0$ and $\beta_{X^{4}}=c$.

There are two minimal measures (up to orthogonal equivalence) of type:

- $(2,1)$ if and only if $\beta_{X}=\beta_{Y}=0$ or $\left(\beta_{X} \beta_{Y} \neq 0\right.$ and $\left.\beta_{X^{4}}=c\right)$.

- $(3,1)$ if and only if $\beta_{X} \beta_{Y} \neq 0$ and $\beta_{X^{4}} \neq c$.

Proof. First note that the pairs $(x, y) \in \mathbb{R}^{2}$ satisfying the equations $x y+y x=0$ and $x^{2}+y^{2}=1$ are

$$
(1,0),(-1,0),(0,1),(0,-1) \in \mathbb{R}^{2} \text {. }
$$

By Lemma 2.2 these are the only pairs in $\mathbb{R}^{2}$ which can be atoms of size 1 in a nc measure for $\beta$.

Claim 1: $\beta$ with $\beta_{X}=\beta_{Y}=0$ and psd $\mathcal{M}_{2}$ admits a measure. Moreover, there are two minimal measures of type $(2,1)$.

Using (6.2) we see that $\mathcal{M}_{2}$ is of the form

$$
\mathcal{M}_{2}=\left(\begin{array}{ccccccc}
1 & 0 & 0 & \beta_{X^{2}} & 0 & 0 & 1-\beta_{X^{2}} \\
0 & \beta_{X^{2}} & 0 & 0 & 0 & 0 & 0 \\
0 & 0 & 1-\beta_{X^{2}} & 0 & 0 & 0 & 0 \\
\beta_{X^{2}} & 0 & 0 & \beta_{X^{4}} & 0 & 0 & \beta_{X^{2}}-\beta_{X^{4}} \\
0 & 0 & 0 & 0 & \beta_{X^{2}}-\beta_{X^{4}} & \beta_{X^{4}}-\beta_{X^{2}} & 0 \\
0 & 0 & 0 & 0 & \beta_{X^{4}}-\beta_{X^{2}} & \beta_{X^{2}}-\beta_{X^{4}} & 0 \\
1-\beta_{X^{2}} & 0 & 0 & \beta_{X^{2}}-\beta_{X^{4}} & 0 & 0 & 1-2 \beta_{X^{2}}+\beta_{X^{4}}
\end{array}\right) .
$$


Let $\mathcal{M}_{2}^{( \pm 1,0)}$ be the moment matrix generated by the atom $( \pm 1,0)$, i.e.,

$$
\mathcal{M}_{2}^{( \pm 1,0)}=\left(\begin{array}{cccc}
1 & \pm 1 & 0 & 1 \\
\pm 1 & 1 & 0 & \pm 1 \\
0 & 0 & 0 & 0 \\
1 & \pm 1 & 0 & 1
\end{array}\right) \bigoplus \mathbf{0}_{3}
$$

We define the matrix function

$$
B(\alpha):=\mathcal{M}_{2}-\alpha\left(\mathcal{M}_{2}^{(1,0)}+\mathcal{M}_{2}^{(-1,0)}\right)
$$

i.e.,

$$
B(\alpha)=\left(\begin{array}{ccccccc}
1-2 \alpha & 0 & 0 & \beta_{X^{2}}-2 \alpha & 0 & 0 & C \\
0 & \beta_{X^{2}}-2 \alpha & 0 & 0 & 0 & 0 & 0 \\
0 & 0 & C & 0 & 0 & 0 & 0 \\
\beta_{X^{2}}-2 \alpha & 0 & 0 & \beta_{X^{4}}-2 \alpha & 0 & 0 & D \\
0 & 0 & 0 & 0 & D & -D & 0 \\
0 & 0 & 0 & 0 & -D & D & 0 \\
C & 0 & 0 & D & 0 & 0 & C-D
\end{array}\right),
$$

where

We have that

$$
C=1-\beta_{X^{2}}, \quad D=\beta_{X^{2}}-\beta_{X^{4}} .
$$

$$
\operatorname{det}\left(\left.B(\alpha)\right|_{\left\{\mathbb{1}, \mathbb{X}, \mathbb{Y}, \mathbb{X}^{2}, \mathbb{X} \mathbb{Y}\right\}}\right)=\operatorname{det}\left(\left.B(\alpha)\right|_{\left\{\mathbb{1}, \mathbb{X}^{2}\right\}}\right) \cdot\left(\beta_{X^{2}}-2 \alpha\right) \cdot C \cdot D,
$$

and

$$
\operatorname{det}\left(\left.B(\alpha)\right|_{\left\{1, \mathbb{X}^{2}\right\}}\right)=\beta_{X^{4}}-\beta_{X^{2}}^{2}+2 \alpha\left(-1+2 \beta_{X^{2}}-\beta_{X^{4}}\right) .
$$

Let $\alpha_{0}>0$ be the smallest positive number such that the rank of $B\left(\alpha_{0}\right)$ is smaller than 5. Note that $B\left(\alpha_{0}\right)$ is psd. By (6.8), (6.9) we get that

$$
\alpha_{0}=\min \left(\frac{\beta_{X^{2}}}{2}, \frac{\beta_{X^{2}}^{2}-\beta_{X^{4}}}{2\left(-1+2 \beta_{X^{2}}-\beta_{X^{4}}\right)}\right) .
$$

If $\alpha_{0}=\frac{\beta_{X^{2}}}{2}$, then $\beta_{X^{4}}-2 \alpha_{0}=\beta_{X^{4}}-\beta_{X}^{2}<0$ by Proposition 6.3 and $B\left(\alpha_{0}\right)$ would not be psd which is a contradiction. Hence

$$
\alpha_{0}=\frac{\beta_{X^{2}}^{2}-\beta_{X^{4}}}{2\left(-1+2 \beta_{X^{2}}-\beta_{X^{4}}\right)} .
$$

The matrix $B\left(\alpha_{0}\right)$ is psd of rank 4 and satisfies the column relations

$$
\mathbb{X}^{2}=\frac{\beta_{X^{2}}-\beta_{X^{4}}}{1-\beta_{X^{2}}} \mathbb{1}, \quad \mathbb{X} \mathbb{Y}+\mathbb{Y} \mathbb{X}=\mathbf{0}, \quad \mathbb{Y}^{2}=\frac{1-2 \beta_{X^{2}}+\beta_{X^{4}}}{1-\beta_{X^{2}}} \mathbb{1} .
$$

By Theorem 3.1 it has a unique (up to orthogonal equivalence) 1-atomic nc measure with an atom $(X, Y) \in\left(\mathbb{S R}^{2 \times 2}\right)^{2}$. Therefore $\mathcal{M}_{2}$ has a minimal measure of type $(2,1)$. Indeed, minimality follows by the following facts:

- Since $\mathcal{M}_{2}$ is a nc moment matrix, there must be at least one atom of size $>1$ in the representing nc measure.

- If there is exactly one atom of size 2 in the representing nc measure, then there must be at least one atom of size 1 , since otherwise $\mathcal{M}_{2}$ would have rank at most 4 . Since $\beta_{X}=\beta_{Y}=0$, atoms $(1,0),(-1,0)$ (resp. $(0,1),(0,-1))$ occur in pairs with the same densities.

By symmetry there exists a unique minimal measure of type $(2,1)$ involving the atoms $(0,1)$ and $(0,-1)$. This concludes the proof of Claim 1.

Claim 2: If $\beta$ admits a nc measure, then it has a representing nc measure with the atoms of size at most 2 .

Claim 2 follows by Proposition 6.1 and Claim 1.

Claim 3: $\beta$ admits a nc measure if and only if (6.7) holds.

A special case $\beta_{X}=\beta_{Y}=0$ of Claim 3 follows by Claim 1. Let us assume that $\beta_{X} \neq 0$ or $\beta_{Y} \neq 0$ and suppose that $\beta$ admits a nc measure. By Claim 2,

$$
\mathcal{M}_{2}=\sum_{i} \lambda_{i} \mathcal{M}_{2}^{\left(x_{i}, y_{i}\right)}+\sum_{j} \xi_{j} \mathcal{M}_{2}^{\left(X_{j}, Y_{j}\right)}
$$


where $\left(x_{i}, y_{i}\right) \in \mathbb{R}^{2},\left(X_{j}, Y_{j}\right) \in \mathbb{S R}^{2 \times 2}, \lambda_{i}>0, \xi_{j}>0$ and $\sum_{i} \lambda_{i}+\sum_{j} \xi_{j}=1$. By Corollary 3.2,

$$
\beta_{X}^{(j)}=\beta_{Y}^{(j)}=\beta_{X^{3}}^{(j)}=\beta_{X^{2} Y}^{(j)}=\beta_{X Y^{2}}^{(j)}=\beta_{Y^{3}}^{(j)}=0,
$$

where $\beta_{w(X, Y)}^{(j)}$ are the moments of $\mathcal{M}_{2}^{\left(X_{j}, Y_{j}\right)}$. By the first paragraph in the proof of Theorem 6.5,

$$
\sum_{i} \lambda_{i} \mathcal{M}_{2}^{\left(x_{i}, y_{i}\right)}=\lambda_{1}^{+} \mathcal{M}_{2}^{(1,0)}+\lambda_{1}^{-} \mathcal{M}_{2}^{(-1,0)}+\lambda_{2}^{+} \mathcal{M}_{2}^{(0,1)}+\lambda_{2}^{-} \mathcal{M}_{2}^{(0,-1)}
$$

where $\lambda_{i}^{ \pm} \geq 0$ for $i=1,2$. Using (6.10), (6.11) and (6.12) we conclude that

$$
\sum_{i} \lambda_{i} \mathcal{M}_{2}^{\left(x_{i}, y_{i}\right)}=\left(\begin{array}{ccccccc}
a & \beta_{X} & \beta_{Y} & b & 0 & 0 & c \\
\beta_{X} & b & 0 & \beta_{X} & 0 & 0 & 0 \\
\beta_{Y} & 0 & a & 0 & 0 & 0 & \beta_{Y} \\
b & \beta_{X} & 0 & b & 0 & 0 & 0 \\
0 & 0 & 0 & 0 & 0 & 0 & 0 \\
0 & 0 & 0 & 0 & 0 & 0 & 0 \\
c & 0 & \beta_{Y} & 0 & 0 & 0 & c
\end{array}\right) \quad \text { for some } a, b, c \geq 0
$$

where

$$
\beta_{X}=\lambda_{1}^{+}-\lambda_{1}^{-}, \quad \beta_{Y}=\lambda_{2}^{+}-\lambda_{2}^{-}, \quad b=\lambda_{1}^{+}+\lambda_{1}^{-}, \quad c=\lambda_{2}^{+}+\lambda_{2}^{-}, \quad a=b+c .
$$

Subclaim 3.1: We have that

$$
\sum_{i} \lambda_{i} \mathcal{M}_{2}^{\left(x_{i}, y_{i}\right)} \succeq\left|\beta_{X}\right| \mathcal{M}_{2}^{\left(\operatorname{sign}\left(\beta_{X}\right) 1,0\right)}+\left|\beta_{Y}\right| \mathcal{M}_{2}^{\left(0, \operatorname{sign}\left(\beta_{Y}\right) 1\right)}=: A,
$$

where $\operatorname{sign}(x)=+$ if $x \geq 0$ and - otherwise.

Since $\beta_{X}=\lambda_{1}^{+}-\lambda_{1}^{-}$and $\lambda_{1}^{ \pm} \geq 0$, it follows that $\lambda_{1}^{\operatorname{sign}\left(\beta_{X}\right)} \geq\left|\operatorname{sign}\left(\beta_{X}\right)\right|$. Thus, (6.13) follows.

$\mathcal{M}_{2}-A$ is of the form

$$
\left(\begin{array}{ccccccc}
1-\left|\beta_{Y}\right|-\left|\beta_{X}\right| & 0 & 0 & \beta_{X^{2}}-\left|\beta_{X}\right| & 0 & 0 & E \\
0 & \beta_{X^{2}}-\left|\beta_{X}\right| & 0 & 0 & 0 & 0 & 0 \\
0 & 0 & E & 0 & 0 & 0 & 0 \\
\beta_{X^{2}}-\left|\beta_{X}\right| & 0 & 0 & \beta_{X^{4}}-\left|\beta_{X}\right| & 0 & 0 & \beta_{X^{2}}-\beta_{X^{4}} \\
0 & 0 & 0 & 0 & \beta_{X^{2}}-\beta_{X^{4}} & -\beta_{X^{2}}+\beta_{X^{4}} & 0 \\
0 & 0 & 0 & 0 & -\beta_{X^{2}}+\beta_{X^{4}} & \beta_{X^{2}}-\beta_{X^{4}} & 0 \\
E & 0 & 0 & \beta_{X^{2}}-\beta_{X^{4}} & 0 & 0 & F
\end{array}\right),
$$

where

By Subclaim 3.1,

$$
E=1-\beta_{X^{2}}-\left|\beta_{Y}\right| \text { and } F=E-\beta_{X^{2}}+\beta_{X^{4}} .
$$

$$
\mathcal{M}_{2}-\sum_{i} \lambda_{i} \mathcal{M}_{2}^{\left(x_{i}, y_{i}\right)} \preceq \mathcal{M}_{2}-A .
$$

Necessary conditions for the existence of a nc measure by Proposition 2.1 and Corollary 2.3 for a nc moment matrix $\mathcal{M}_{2}-A$ are that $\mathcal{M}_{2}-A$ is psd and $\left.\left(\mathcal{M}_{2}-A\right)\right|_{\{1, \mathbb{X}, \mathbb{Y}, \mathbb{X Y}\}}$ is pd. The latter is equivalent to

(6.14) $1-\left|\beta_{Y}\right|-\left|\beta_{X}\right|>0, \quad \beta_{X^{2}}-\left|\beta_{X}\right|>0, \quad 1-\beta_{X^{2}}-\left|\beta_{Y}\right|>0, \quad \beta_{X^{2}}-\beta_{X^{4}}>0, \quad \operatorname{det}\left(\left.\mathcal{M}_{2}\right|_{\left\{\mathbb{1}, \mathbb{X}^{2}\right\}}\right) \geq 0$.

Further on, this system is equivalent to the conditions (6.7). This proves Claim 3.

Claim 4: Minimal measures are as stated in the theorem.

If $\beta_{X}=\beta_{Y}=0$, Claim 4 follows by Claim 1. Suppose $\beta_{X} \neq 0$ or $\beta_{Y} \neq 0$. Let $A$ be as in the proof of Claim 3 . The following statements are true:

(1) Minimal measure is unique of type $(1,1)$ if and only if the rank of $\mathcal{M}_{2}-A$ is 4 and one of the moments $\beta_{X}$, $\beta_{Y}$ is 0 .

(2) Minimal measure is unique of type $(2,1)$ if and only if

(a) the rank of $\mathcal{M}_{2}-A$ is 4 and $\beta_{X} \beta_{Y} \neq 0$. 
(b) the rank of $\mathcal{M}_{2}-A$ is 5 and one of the moments $\beta_{X}, \beta_{Y}$ is 0 in which case we subtract $\alpha\left(\mathcal{M}_{2}^{(1,0)}+\mathcal{M}_{2}^{(-1,0)}\right)$ or $\alpha\left(\mathcal{M}_{2}^{(0,1)}+\mathcal{M}_{2}^{(0,-1)}\right)$ with the smallest $\alpha>0$ such that the rank falls to 4 .

(3) There are two minimal measures of type (3,1) if and only if the rank of $\mathcal{M}_{2}-A$ is 5 and $\beta_{X} \beta_{Y} \neq 0$ in which case we subtract $\alpha\left(\mathcal{M}_{2}^{(1,0)}+\mathcal{M}_{2}^{(-1,0)}\right)$ or $\alpha\left(\mathcal{M}_{2}^{(0,1)}+\mathcal{M}_{2}^{(0,-1)}\right)$ with the smallest $\alpha>0$ such that the rank falls to 4 .

The rank of $\mathcal{M}_{2}-A$ is 5 exactly when in (6.14) we have $\operatorname{det}\left(\left.\mathcal{M}_{2}\right|_{\left\{\mathbb{1}, \mathbb{X}^{2}\right\}}\right)>0$ which is exactly when $c<\beta_{X^{4}}$ with $c$ as in the statement of the theorem.

6.2. Pair $\mathbb{X} \mathbb{Y}+\mathbb{Y} \mathbb{X}=\mathbf{0}$ and $\mathbb{Y}^{2}=\mathbb{1}$. In this subsection we study a nc sequence $\beta$ with a moment matrix $\mathcal{M}_{2}$ of rank 5 satisfying the relations $\mathbb{X} \mathbb{Y}+\mathbb{Y} \mathbb{X}=0$ and $\mathbb{Y}^{2}=\mathbb{1}$. In Theorem 6.8 we characterize exactly when $\beta$ admits a nc measure. Moreover, we classify type and uniqueness of the minimal measure.

The form of $\mathcal{M}_{2}$ is given by the following proposition.

Proposition 6.6. Suppose $\beta \equiv \beta^{(4)}$ is a nc sequence with a moment matrix $\mathcal{M}_{2}$ of rank 5 satisfying the relations

$$
\mathbb{X} \mathbb{Y}+\mathbb{Y}=\mathbf{0} \text { and } \mathbb{Y}^{2}=\mathbb{1}
$$

Then $\mathcal{M}_{2}$ is of the form

$$
\left(\begin{array}{ccccccc}
\beta_{1} & 0 & \beta_{Y} & \beta_{X^{2}} & 0 & 0 & \beta_{1} \\
0 & \beta_{X^{2}} & 0 & \beta_{X^{3}} & 0 & 0 & 0 \\
\beta_{Y} & 0 & \beta_{1} & 0 & 0 & 0 & \beta_{Y} \\
\beta_{X^{2}} & \beta_{X^{3}} & 0 & \beta_{X^{4}} & 0 & 0 & \beta_{X^{2}} \\
0 & 0 & 0 & 0 & \beta_{X^{2}} & -\beta_{X^{2}} & 0 \\
0 & 0 & 0 & 0 & -\beta_{X^{2}} & \beta_{X^{2}} & 0 \\
\beta_{1} & 0 & \beta_{Y} & \beta_{X^{2}} & 0 & 0 & \beta_{1}
\end{array}\right) .
$$

Proof. The relations (6.15) give us the following system in $\mathcal{M}_{2}$

$$
\begin{array}{rrr}
2 \beta_{X Y}=0, & \beta_{X^{2} Y^{2}}+\beta_{X Y X Y}=0, & \beta_{Y^{3}}=\beta_{Y}, \\
2 \beta_{X^{2} Y}=0, & 2 \beta_{X Y^{3}}=0, & \beta_{X^{2} Y^{2}}=\beta_{X^{2}}, \\
2 \beta_{X Y^{2}}=0, & \beta_{Y^{2}}=\beta_{1}, & \beta_{X Y^{3}}=\beta_{X Y}, \\
2 \beta_{X^{3} Y}=0, & \beta_{X Y^{2}}=\beta_{X}, & \beta_{Y^{4}}=\beta_{Y^{2}} .
\end{array}
$$

The solution to (6.17) is given by

$$
\begin{aligned}
\beta_{X Y}=0, & \beta_{X Y X Y}=-\beta_{X^{2}}, \\
\beta_{X^{2} Y}=0, & \beta_{Y^{4}}=\beta_{Y^{2}}=\beta_{1}, \\
\beta_{X Y^{2}}=\beta_{X}=0, & \beta_{Y^{3}}=\beta_{Y}, \\
\beta_{X^{3} Y}=\beta_{X Y^{3}}=0, & \beta_{X^{2} Y^{2}}=\beta_{X^{2}},
\end{aligned}
$$

and thus $\mathcal{M}_{2}$ takes the form (6.16).

Proposition 6.7. Suppose $\beta \equiv \beta^{(4)}$ is a normalized nc sequence with a moment matrix $\mathcal{M}_{2}$ of rank 5 satisfying the relations $\mathbb{X} \mathbb{Y}+\mathbb{Y} \mathbb{X}=0$ and $\mathbb{Y}^{2}=\mathbb{1}$. Then $\mathcal{M}_{2}$ is positive semidefinite if and only if

$$
\beta_{X^{3}} \in \mathbb{R}, \quad \beta_{X^{2}}>0, \quad\left|\beta_{Y}\right|<1, \quad \beta_{X^{4}}>\frac{\beta_{X^{2}}^{3}+\beta_{X^{3}}^{2}-\beta_{Y}^{2} \beta_{X^{3}}^{2}}{\left(1-\beta_{Y}^{2}\right) \beta_{X^{2}}}=: c .
$$

Proof. Write $\mathcal{M}_{2}$ as $\mathcal{M}_{2}=\left(\begin{array}{cc}A & B \\ B^{t} & C\end{array}\right)$ where

$$
A=\left.\mathcal{M}_{2}\right|_{\left\{\mathbb{1}, \mathbb{X}, \mathbb{Y}, \mathbb{X}^{2}, \mathbb{X} \mathbb{Y}\right\}}, \quad B=\left.\mathcal{M}_{2}\right|_{\left\{\mathbb{1}, \mathbb{X}, \mathbb{Y}, \mathbb{X}^{2}, \mathbb{X} \mathbb{Y}\right\},\left\{\mathbb{X} \mathbb{X}, \mathbb{Y}^{2}\right\}} \quad C=\left.\mathcal{M}_{2}\right|_{\left\{\mathbb{Y X}, \mathbb{Y}^{2}\right\}} .
$$

Since $\mathcal{M}_{2}$ satisfies the relations $\mathbb{X} \mathbb{Y}+\mathbb{Y} \mathbb{X}=\mathbf{0}$ and $\mathbb{Y}^{2}=\mathbb{1}$, it follows that $\operatorname{rank}\left(\mathcal{M}_{2}\right)=\operatorname{rank}(A)=5$. Now by Lemma 6.4, $\mathcal{M}_{2}$ is psd if and only if $A$ is psd. Since $\operatorname{rank}(A)=5, A$ is psd if and only if $A$ is positive definite (pd). By Sylvester's criterion $A$ is pd if and only if all its principal minors are positive. 
First we will prove that the conditions in (6.19) are necessary for $A$ being pd. We have that $\left.A\right|_{\{\mathbb{X}\}}=\beta_{X^{2}}>0$, $\left.A\right|_{\{1, \mathbb{Y}\}}=1-\beta_{Y}^{2}>0$ (if and only if $\left.1>\left|\beta_{Y}\right|\right)$. Finally, $\operatorname{det}\left(\left.A\right|_{\left\{\mathbb{1}, \mathbb{X}, \mathbb{Y}, \mathbb{X}^{2}\right\}}\right)>0$ implies that $\beta_{X^{4}}>c$.

It remains to prove that the conditions in (6.19) are sufficient for $A$ being pd. The principal minors $\alpha_{i}, i=1, \ldots, 5$, of $A$ are

$$
\alpha_{1}=1, \quad \alpha_{2}=\beta_{X^{2}}, \quad \alpha_{3}=\beta_{X^{2}}\left(1-\beta_{Y}^{2}\right), \quad \alpha_{4}=-\beta_{X^{2}}^{3}-\beta_{X^{3}}^{2}+\beta_{Y}^{2} \beta_{X^{3}}^{2}+\beta_{X^{4}} \beta_{X^{2}}\left(1-\beta_{Y}^{2}\right), \quad \alpha_{5}=\alpha_{4} \beta_{X^{2}} .
$$

Now, if (6.19) is true, then clearly $\alpha_{1}, \alpha_{2}, \alpha_{3}>0$ and $\alpha_{5}>0$ if $\alpha_{4}>0$. Note that $\alpha_{4}>0$ if and only if $\beta_{X^{4}}>c$ which concludes the proof of the proposition.

The following theorem characterizes normalized nc sequences $\beta$ with a moment matrix $\mathcal{M}_{2}$ of rank 5 satisfying the relations $\mathbb{X} \mathbb{Y}+\mathbb{Y} \mathbb{X}=\mathbf{0}$ and $\mathbb{Y}^{2}=\mathbb{1}$, which admit a nc measure.

Theorem 6.8. Suppose $\beta \equiv \beta^{(4)}$ is a normalized nc sequence with a moment matrix $\mathcal{M}_{2}$ of rank 5 satisfying the relations $\mathbb{X} \mathbb{Y}+\mathbb{Y} \mathbb{X}=0$ and $\mathbb{Y}^{2}=\mathbb{1}$. Then $\beta$ admits a nc measure exactly in the following cases:

(1) $\mathcal{M}_{2}$ is positive semidefinite and $\beta_{X^{3}}=\beta_{Y}=0$. The minimal measure is unique (up to orthogonal equivalence) and of type $(2,1)$.

(2) $\mathcal{M}_{2}$ is positive semidefinite and

$$
\beta_{X^{3}}=0, \beta_{X^{2}}>0,0<\left|\beta_{Y}\right|<1, \beta_{X^{4}} \geq \frac{\beta_{X^{2}}^{2}}{1-\left|\beta_{Y}\right|} .
$$

Moreover, assume that (6.20) holds. The minimal measure is unique (up to orthogonal equivalence). It is of type $(1,1)$ if and only if $\beta_{X^{4}}=\frac{\beta_{X}^{2}}{1-\left|\beta_{Y}\right|}$. Otherwise it is of type $(2,1)$.

Proof. First note that the pairs $(x, y) \in \mathbb{R}^{2}$ satisfying the equations $x y+y x=0$ and $y^{2}=1$ are

$$
(0,1),(0,-1) \in \mathbb{R}^{2} \text {. }
$$

By Lemma 2.2 these are the only pairs from $\mathbb{R}^{2}$ which can be atoms of size 1 in a nc measure for $\beta$.

Claim 1: $\beta$ with $\beta_{X^{3}}=\beta_{Y}=0$ and psd $\mathcal{M}_{2}$ admits a nc measure. Moreover, the minimal measure is unique and of type $(2,1)$.

Using (6.16) we see that $\mathcal{M}_{2}$ is of the form

$$
\mathcal{M}_{2}=\left(\begin{array}{ccccccc}
1 & 0 & 0 & \beta_{X^{2}} & 0 & 0 & 1 \\
0 & \beta_{X^{2}} & 0 & 0 & 0 & 0 & 0 \\
0 & 0 & 1 & 0 & 0 & 0 & 0 \\
\beta_{X^{2}} & 0 & 0 & \beta_{X^{4}} & 0 & 0 & \beta_{X^{2}} \\
0 & 0 & 0 & 0 & \beta_{X^{2}} & -\beta_{X^{2}} & 0 \\
0 & 0 & 0 & 0 & -\beta_{X^{2}} & \beta_{X^{2}} & 0 \\
1 & 0 & 0 & \beta_{X^{2}} & 0 & 0 & 1
\end{array}\right)
$$

Let $\mathcal{M}_{2}^{(0, \pm 1)}$ be the moment matrix generated by the atom $(0, \pm 1)$, i.e.,

$$
\mathcal{M}_{2}^{(0, \pm 1)}=\left(\begin{array}{ccccccc}
1 & 0 & \pm 1 & 0 & 0 & 0 & 1 \\
0 & 0 & 0 & 0 & 0 & 0 & 0 \\
\pm 1 & 0 & 1 & 0 & 0 & 0 & \pm 1 \\
0 & 0 & 0 & 0 & 0 & 0 & 0 \\
0 & 0 & 0 & 0 & 0 & 0 & 0 \\
0 & 0 & 0 & 0 & 0 & 0 & 0 \\
1 & 0 & \pm 1 & 0 & 0 & 0 & 1
\end{array}\right)
$$

We define the matrix function

$$
B(\alpha):=\mathcal{M}_{2}-\alpha\left(\mathcal{M}_{2}^{(0,1)}+\mathcal{M}_{2}^{(0,-1)}\right),
$$

i.e.,

$$
B(\alpha)=\left(\begin{array}{ccccccc}
1-2 \alpha & 0 & 0 & \beta_{X^{2}} & 0 & 0 & 1-2 \alpha \\
0 & \beta_{X^{2}} & 0 & 0 & 0 & 0 & 0 \\
0 & 0 & 1-2 \alpha & 0 & 0 & 0 & 0 \\
\beta_{X^{2}} & 0 & 0 & \beta_{X^{4}} & 0 & 0 & \beta_{X^{2}} \\
0 & 0 & 0 & 0 & \beta_{X^{2}} & -\beta_{X^{2}} & 0 \\
0 & 0 & 0 & 0 & -\beta_{X^{2}} & \beta_{X^{2}} & 0 \\
1-2 \alpha & 0 & 0 & \beta_{X^{2}} & 0 & 0 & 1-2 \alpha
\end{array}\right) .
$$


We have

$$
\operatorname{det}\left(\left.B(\alpha)\right|_{\left\{\mathbb{1}, \mathbb{X}, \mathbb{Y}, \mathbb{X}^{2}, \mathbb{X} \mathbb{Y}\right\}}\right)=\operatorname{det}\left(\left.B(\alpha)\right|_{\left\{\mathbb{1}, \mathbb{X}^{2}\right\}}\right) \cdot \beta_{X^{2}}^{2} \cdot(1-2 \alpha)
$$

and

$$
\operatorname{det}\left(\left.B(\alpha)\right|_{\left\{\mathbb{1}, \mathbb{X}^{2}\right\}}\right)=\beta_{X^{4}}-\beta_{X^{2}}^{2}-2 \alpha \beta_{X^{4}} .
$$

Let $\alpha_{0}>0$ be the smallest positive number such that the rank of $B\left(\alpha_{0}\right)$ is smaller than 5. By (6.21) and (6.22) we get that

$$
\alpha_{0}=\min \left(\frac{1}{2}, \frac{\beta_{X^{4}}-\beta_{X^{2}}^{2}}{2 \beta_{X^{4}}}\right)=\frac{1}{2}-\frac{\beta_{X^{2}}^{2}}{2 \beta_{X^{4}}} .
$$

The matrix $B\left(\alpha_{0}\right)$ is psd matrix of rank 4 and satisfies the relations

$$
\mathbb{X}^{2}=\frac{\beta_{X^{4}}}{\beta_{X^{2}}} \mathbb{1}, \quad \mathbb{X} \mathbb{Y}+\mathbb{Y} \mathbb{X}=\mathbf{0}, \quad \mathbb{Y}^{2}=\mathbb{1} .
$$

By Theorem 3.1 it has a unique (up to orthogonal equivalence) 1-atomic measure with an atom $(X, Y) \in\left(\mathbb{S R}^{2 \times 2}\right)^{2}$. Therefore $\mathcal{M}_{2}$ has a minimal measure of type $(2,1)$. Indeed, minimality follows by the following facts:

- Since $\mathcal{M}_{2}$ is a nc moment matrix, there must be at least one atom of size $>1$ in the representing measure.

- If there is exactly one atom of size 2 in the representing measure, then there must be at least one atom of size 1 , since otherwise $\mathcal{M}_{2}$ would have rank at most 4 . Since $\beta_{X}=\beta_{Y}=0$, atoms $(0,1),(0,-1)$ occur in pairs with the same densities.

Claim 2: If $\beta$ admits a measure, then it has a representing measure with the atoms of size at most 2 .

Claim 2 follows by Proposition 6.1 and Claim 1.

Claim 3: If $\beta_{x^{3}} \neq 0$ or $\beta_{Y} \neq 0$, then $\beta$ admits a nc measure if and only if (6.20) holds.

Let us assume that $\beta_{x^{3}} \neq 0$ or $\beta_{Y} \neq 0$ and suppose that $\beta$ admits a nc measure. By Claim 2,

$$
\mathcal{M}_{2}=\sum_{i} \lambda_{i} \mathcal{M}_{2}^{\left(x_{i}, y_{i}\right)}+\sum_{j} \xi_{j} \mathcal{M}_{2}^{\left(X_{j}, Y_{j}\right)} \text {. }
$$

where $\left(x_{i}, y_{i}\right) \in \mathbb{R}^{2},\left(X_{j}, Y_{j}\right) \in \mathbb{S R}^{2 \times 2}, \lambda_{i}>0, \xi_{j}>0$ and $\sum_{i} \lambda_{i}+\sum_{j} \xi_{j}=1$. By Corollary 3.2,

$$
\beta_{X}^{(j)}=\beta_{Y}^{(j)}=\beta_{X^{3}}^{(j)}=\beta_{X^{2} Y}^{(j)}=\beta_{X Y^{2}}^{(j)}=\beta_{Y^{3}}^{(j)}=0,
$$

where $\beta_{w(X, Y)}^{(j)}$ are the moments of $\mathcal{M}_{2}^{\left(X_{j}, Y_{j}\right)}$. By the first paragraph in the proof of Theorem 6.8 , it follows that

$$
\sum_{i} \lambda_{i} \mathcal{M}_{2}^{\left(x_{i}, y_{i}\right)}=\lambda_{+} \mathcal{M}_{2}^{(0,1)}+\lambda_{-} \mathcal{M}_{2}^{(0,-1)}
$$

where $\lambda_{ \pm} \geq 0$. Using (6.23), (6.24) and (6.25) we conclude that $\sum_{i} \lambda_{i} \mathcal{M}_{2}^{\left(x_{i}, y_{i}\right)}$ is of the form

$$
\sum_{i} \lambda_{i} \mathcal{M}_{2}^{\left(x_{i}, y_{i}\right)}=\left(\begin{array}{ccccccc}
a & 0 & \beta_{Y} & 0 & 0 & 0 & a \\
0 & 0 & 0 & 0 & 0 & 0 & 0 \\
\beta_{Y} & 0 & a & 0 & 0 & 0 & \beta_{Y} \\
0 & 0 & 0 & 0 & 0 & 0 & 0 \\
0 & 0 & 0 & 0 & 0 & 0 & 0 \\
0 & 0 & 0 & 0 & 0 & 0 & 0 \\
a & 0 & \beta_{Y} & 0 & 0 & 0 & a
\end{array}\right) \quad \text { for some } a \geq 0
$$

where

$$
\beta_{Y}=\lambda_{+}-\lambda_{-}, \quad a=\lambda_{+}+\lambda_{-} .
$$

Subclaim 3.1: If $\beta$ has a measure, then $\beta_{X^{3}}=0$.

Combining (6.23), (6.24) and (6.26), the subclaim follows.

Subclaim 3.2: We have that

$$
\sum_{i} \lambda_{i} \mathcal{M}_{2}^{\left(x_{i}, y_{i}\right)} \succeq\left|\beta_{Y}\right| \mathcal{M}^{\left(0, \operatorname{sign}\left(\beta_{Y}\right) 1\right)}=: A,
$$


where $\operatorname{sign}\left(\beta_{Y}\right)=+$ if $\beta_{Y} \geq 0$ and -1 otherwise.

Since $\beta_{Y}=\lambda_{+}-\lambda_{-}$and $\lambda_{ \pm} \geq 0$, it follows that $\lambda_{\operatorname{sign}\left(\beta_{Y}\right)} \geq\left|\operatorname{sign}\left(\beta_{Y}\right)\right|$. Thus, (6.27) follows.

$\mathcal{M}_{2}-A$ is of the form

$$
\left(\begin{array}{ccccccc}
1-\left|\beta_{Y}\right| & 0 & 0 & \beta_{X^{2}} & 0 & 0 & 1-\left|\beta_{Y}\right| \\
0 & \beta_{X^{2}} & 0 & 0 & 0 & 0 & 0 \\
0 & 0 & 1-\left|\beta_{Y}\right| & 0 & 0 & 0 & 0 \\
\beta_{X^{2}} & 0 & 0 & \beta_{X^{4}} & 0 & 0 & \beta_{X^{2}} \\
0 & 0 & 0 & 0 & \beta_{X^{2}} & -\beta_{X^{2}} & 0 \\
0 & 0 & 0 & 0 & -\beta_{X^{2}} & \beta_{X^{2}} & 0 \\
1-\left|\beta_{Y}\right| & 0 & 0 & \beta_{X^{2}} & 0 & 0 & 1-\left|\beta_{Y}\right|
\end{array}\right)
$$

By Subclaim 3.2, $\mathcal{M}_{2}-\sum_{i} \lambda_{i} \mathcal{M}_{2}^{\left(x_{i}, y_{i}\right)} \succeq \mathcal{M}_{2}-A$. Necessary conditions for the existence of a nc measure by Proposition 2.1 and Corollary 2.3 for a nc moment matrix $\mathcal{M}_{2}-A$ are that $\mathcal{M}_{2}-A$ is psd and $\left.\left(\mathcal{M}_{2}-A\right)\right|_{\{\mathbb{1}, \mathbb{X}, \mathbb{Y}, \mathbb{X}\}}$ is $\mathrm{pd}$. The latter is equivalent to

$$
1-\left|\beta_{Y}\right|>0, \quad \beta_{X^{2}}>0, \quad \operatorname{det}\left(\left.\mathcal{M}_{2}\right|_{\left\{1, \mathbb{X}^{2}\right\}}\right) \geq 0 .
$$

Further on, this system is equivalent to the conditions (6.20). This proves Claim 3.

Claim 4: Minimal measures are as stated in the theorem.

If $\beta_{X^{3}}=\beta_{Y}=0$, Claim 4 follows by Claim 1. Suppose $\beta_{Y} \neq 0$. Let $A$ be as in Subclaim 3.1. The following statements are true:

(1) Minimal measure is unique of type $(1,1)$ if and only if the rank of $\mathcal{M}_{2}-A \succeq 0$ is 4 .

(2) Minimal measure is unique of type $(2,1)$ if and only if the rank of $\mathcal{M}_{2}-A \succeq 0$ is 5 in which case we subtract $\alpha\left(\mathcal{M}_{2}^{(0,1)}+\mathcal{M}_{2}^{(0,-1)}\right)$ with the smallest $\alpha>0$ such that the rank falls to 4.

The rank of $\mathcal{M}_{2}-A$ is 5 exactly when in (6.28) we have $\operatorname{det}\left(\left.\mathcal{M}_{2}\right|_{\left\{\mathbb{1}, \mathbb{X}^{2}\right\}}\right)>0$ which is exactly when $\beta_{X^{4}}>$ $\frac{\beta_{X^{2}}^{2}}{1-\left|\beta_{Y}\right|}$.

6.3. Pair $\mathbb{X} \mathbb{Y}+\mathbb{Y} \mathbb{X}=0$ and $\mathbb{Y}^{2}-\mathbb{X}^{2}=\mathbb{1}$. In this subsection we study a nc sequence $\beta$ with a moment matrix $\mathcal{M}_{2}$ of rank 5 satisfying the relations $\mathbb{X} \mathbb{Y}+\mathbb{Y} \mathbb{X}=0$ and $\mathbb{Y}^{2}+\mathbb{X}^{2}=\mathbb{1}$. In Theorem 6.11 we characterize exactly when $\beta$ admits a nc measure. Moreover, we classify the type and uniqueness of the minimal measure.

The form of $\mathcal{M}_{2}$ is given by the following proposition.

Proposition 6.9. Suppose $\beta \equiv \beta^{(4)}$ is a nc sequence with a moment matrix $\mathcal{M}_{2}$ of rank 5 satisfying the relations

$$
\mathbb{X} \mathbb{Y}+\mathbb{Y}=\mathbf{0} \text { and } \mathbb{Y}^{2}-\mathbb{X}^{2}=\mathbb{1}
$$

Then $\mathcal{M}_{2}$ is of the form

$$
\left(\begin{array}{ccccccc}
\beta_{1} & \beta_{X} & \beta_{Y} & \beta_{X^{2}} & 0 & 0 & \beta_{1}+\beta_{X^{2}} \\
\beta_{X} & \beta_{X^{2}} & 0 & -\beta_{X} & 0 & 0 & 0 \\
\beta_{Y} & 0 & \beta_{1}+\beta_{X^{2}} & 0 & 0 & 0 & \beta_{Y} \\
\beta_{X^{2}} & -\beta_{X} & 0 & \beta_{X^{4}} & 0 & 0 & \beta_{X^{2}}+\beta_{X^{4}} \\
0 & 0 & 0 & 0 & \beta_{X^{2}}+\beta_{X^{4}} & -\beta_{X^{2}}-\beta_{X^{4}} & 0 \\
0 & 0 & 0 & 0 & -\beta_{X^{2}}-\beta_{X^{4}} & \beta_{X^{2}}+\beta_{X^{4}} & 0 \\
\beta_{1}+\beta_{X^{2}} & 0 & \beta_{Y} & \beta_{X^{2}}+\beta_{X^{4}} & 0 & 0 & \beta_{1}+2 \beta_{X^{2}}+\beta_{X^{4}}
\end{array}\right) .
$$

Proof. The relations (6.1) give us the following system in $\mathcal{M}_{2}$

$$
\begin{array}{rrr}
2 \beta_{X Y}=0, & \beta_{X^{2} Y^{2}}+\beta_{X Y X Y}=0, & \beta_{Y^{3}}=\beta_{Y}+\beta_{X^{2} Y}, \\
2 \beta_{X^{2} Y}=0, & 2 \beta_{X Y^{3}}=0, & \beta_{X^{2} Y^{2}}=\beta_{X^{2}}+\beta_{X^{4}}, \\
2 \beta_{X Y^{2}}=0, & \beta_{Y^{2}}=\beta_{1}+\beta_{X^{2}}, & \beta_{X Y^{3}}=\beta_{X Y}+\beta_{X^{3} Y}, \\
2 \beta_{X^{3} Y}=0, & \beta_{X Y^{2}}=\beta_{X}+\beta_{X^{3}}, & \beta_{Y^{4}}=\beta_{Y^{2}}+\beta_{X^{2} Y^{2}} .
\end{array}
$$

The solution to (6.31) is given by 


$$
\begin{aligned}
& \beta_{X}=-\beta_{X^{3}}, \quad \beta_{X^{4}}=\beta_{1}+2 \beta_{X^{2}}+\beta_{X^{4}}, \\
& \beta_{X Y X Y}=-\beta_{X^{4}}-\beta_{X^{2}}, \quad \beta_{Y^{3}}=\beta_{Y}, \\
& \beta_{X Y}=\beta_{X^{2} Y}=\beta_{X Y^{2}}=\beta_{X^{3} Y}=\beta_{X Y^{3}}=0, \quad \beta_{X^{2} Y^{2}}=\beta_{X^{2}}+\beta_{X^{4}},
\end{aligned}
$$

and thus $\mathcal{M}_{2}$ takes the form (6.30).

Proposition 6.10. Suppose $\beta \equiv \beta^{(4)}$ is a nc sequence with a moment matrix $\mathcal{M}_{2}$ of rank 5 satisfying the relations $\mathbb{X} \mathbb{Y}+\mathbb{Y} \mathbb{X}=\mathbf{0}$ and $\mathbb{Y}^{2}-\mathbb{X}^{2}=\mathbb{1}$. Then $\mathcal{M}_{2}$ is positive semidefinite if and only if

$$
0 \leq\left|\beta_{X}\right|<\sqrt{\beta_{X^{2}}}, \quad\left|\beta_{Y}\right|<c, \quad d<\beta_{X^{4}},
$$

where

$$
\begin{aligned}
c & :=\sqrt{\frac{\left(1+\beta_{X^{2}}\right)\left(\beta_{X^{2}}-\beta_{X}^{2}\right)}{\beta_{X^{2}}}}, \\
d & :=\frac{\beta_{X^{2}}^{3}+\beta_{X^{2}}^{4}+\beta_{X}^{2}-\beta_{Y}^{2} \beta_{X}^{2}+3 \beta_{X^{2}} \beta_{X}^{2}+2 \beta_{X^{2}}^{2} \beta_{X}^{2}}{\left(1+\beta_{X^{2}}\right)\left(\beta_{X^{2}}-\beta_{X}^{2}\right)-\beta_{Y}^{2} \beta_{X^{2}}} .
\end{aligned}
$$

Proof. Write $\mathcal{M}_{2}$ as $\mathcal{M}_{2}=\left(\begin{array}{cc}A & B \\ B^{t} & C\end{array}\right)$ where

$$
A=\left.\mathcal{M}_{2}\right|_{\left\{1, \mathbb{X}, \mathbb{Y}, \mathbb{X}^{2}, \mathbb{X} \mathbb{Y}\right\}}, \quad B=\left.\mathcal{M}_{2}\right|_{\left\{\mathbb{1}, \mathbb{X}, \mathbb{Y}, \mathbb{X}^{2}, \mathbb{X} \mathbb{Y}\right\},\left\{\mathbb{Y X}, \mathbb{Y}^{2}\right\}} \quad C=\left.\mathcal{M}_{2}\right|_{\left\{\mathbb{Y X}, \mathbb{Y}^{2}\right\}} .
$$

Since $\mathcal{M}_{2}$ satisfies the relations $\mathbb{X} \mathbb{Y}+\mathbb{Y} \mathbb{X}=\mathbf{0}$ and $\mathbb{Y}^{2}-\mathbb{X}^{2}=\mathbb{1}$, it follows that $\operatorname{rank}\left(\mathcal{M}_{2}\right)=\operatorname{rank}(A)=5$. Now by Lemma 6.4, $\mathcal{M}_{2}$ is psd if and only if $A$ is psd. Since $\operatorname{rank}(A)=5, A$ is psd if and only if $A$ is positive definite (pd). By Sylvester's criterion $A$ is pd if and only if all its principal minors are positive. The principal minors $\alpha_{i}, i=1, \ldots, 5$, of $A$ are

$$
\begin{aligned}
& \alpha_{1}=1, \quad \alpha_{2}=\beta_{X^{2}}-\beta_{X}^{2}, \quad \alpha_{3}=\left(1+\beta_{X^{2}}\right) \alpha_{2}-\beta_{Y}^{2} \beta_{X^{2}}, \\
& \alpha_{4}=\beta_{X^{4}} \alpha_{3}-\beta_{X^{2}}^{3}+\beta_{X^{2}}^{4}+\beta_{X}^{2}-\beta_{Y}^{2} \beta_{X}^{2}+3 \beta_{X^{2}} \beta_{X}^{2}+2 \beta_{X^{2}}^{2} \beta_{X}^{2}, \quad \alpha_{5}=\alpha_{4}\left(\beta_{X^{2}}+\beta_{X^{4}}\right) .
\end{aligned}
$$

Thus

$$
\alpha_{2}>0 \Leftrightarrow 0 \leq\left|\beta_{X}\right|<\sqrt{\beta_{X^{2}}}, \quad \alpha_{3}>0 \Leftrightarrow\left|\beta_{Y}\right|<c, \quad \alpha_{4}>0 \Leftrightarrow d<\beta_{X^{4}} .
$$

Note that $\alpha_{2}, \alpha_{3}, \alpha_{4}>0$ also imply $\alpha_{5}>0$ (since $\beta_{X^{4}}>0$ if $\left.A\right|_{\left\{\mathbb{1}, \mathbb{X}, \mathbb{Y}, \mathbb{X}^{2}\right\}}$ is pd). This proves the proposition.

The following theorem characterizes normalized nc sequences $\beta$ with a moment matrix $\mathcal{M}_{2}$ of rank 5 satisfying the relations $\mathbb{X} \mathbb{Y}+\mathbb{Y} \mathbb{X}=\mathbf{0}$ and $\mathbb{Y}^{2}-\mathbb{X}^{2}=\mathbb{1}$, which admit a nc measure.

Theorem 6.11. Suppose $\beta \equiv \beta^{(4)}$ is a nc sequence with a moment matrix $\mathcal{M}_{2}$ of rank 5 satisfying the relations $\mathbb{X} \mathbb{Y}+\mathbb{Y} \mathbb{X}=\mathbf{0}$ and $\mathbb{Y}^{2}-\mathbb{X}^{2}=\mathbb{1}$. Then $\beta$ admits a nc measure exactly in the following cases:

(1) $\mathcal{M}_{2}$ is positive semidefinite and $\beta_{X}=\beta_{Y}=0$. The minimal measure is unique (up to orthogonal equivalence) and of type $(2,1)$.

(2) $\mathcal{M}_{2}$ is positive semidefinite and

$$
\beta_{X}=0,0<\left|\beta_{Y}\right|<1, \beta_{X^{4}} \geq \frac{\beta_{X^{2}}^{2}}{1-\left|\beta_{Y}\right|} .
$$

Moreover, assume that (6.35) holds. The minimal measure is unique (up to orthogonal equivalence). It is of type $(1,1)$ if and only if $\beta_{X^{4}}=\frac{\beta_{X^{2}}}{1-\left|\beta_{Y}\right|}$. Otherwise it is of type $(2,1)$.

Proof. First note that the pairs $(x, y) \in \mathbb{R}^{2}$ satisfying the equations $x y+y x=0$ and $y^{2}-x^{2}=1$ are

$$
(0,1),(0,-1) \in \mathbb{R}^{2} \text {. }
$$

By Lemma 2.2 these are the only pairs from $\mathbb{R}^{2}$ which can be atoms of size 1 in a nc measure of $\beta$.

Claim 1: $\beta$ with $\beta_{X}=\beta_{Y}=0$ and psd $\mathcal{M}_{2}$ admits a nc measure. Moreover, the minimal measure is unique and of type $(2,1)$. 
Using (6.16) we see that $\mathcal{M}_{2}$ is of the form

$$
\mathcal{M}_{2}=\left(\begin{array}{ccccccc}
1 & 0 & 0 & \beta_{X^{2}} & 0 & 0 & 1+\beta_{X^{2}} \\
0 & \beta_{X^{2}} & 0 & 0 & 0 & 0 & 0 \\
0 & 0 & 1+\beta_{X^{2}} & 0 & 0 & 0 & 0 \\
\beta_{X^{2}} & 0 & 0 & \beta_{X^{4}} & 0 & 0 & \beta_{X^{2}}+\beta_{X^{4}} \\
0 & 0 & 0 & 0 & \beta_{X^{2}}+\beta_{X^{4}} & -\beta_{X^{2}}-\beta_{X^{4}} & 0 \\
0 & 0 & 0 & 0 & -\beta_{X^{2}}-\beta_{X^{4}} & \beta_{X^{2}}+\beta_{X^{4}} & 0 \\
1+\beta_{X^{2}} & 0 & 0 & \beta_{X^{2}}+\beta_{X^{4}} & 0 & 0 & 1+2 \beta_{X^{2}}+\beta_{X^{4}}
\end{array}\right) .
$$

Let $\mathcal{M}_{2}^{(0,1)}, \mathcal{M}_{2}^{(0,-1)}$ be as in the proof of Theorem 6.5. We define the matrix function

$$
B(\alpha):=\mathcal{M}_{2}-\alpha\left(\mathcal{M}_{2}^{(0,1)}+\mathcal{M}_{2}^{(0,-1)}\right),
$$

i.e.,

$$
B(\alpha)=\left(\begin{array}{ccccccc}
1-2 \alpha & 0 & 0 & \beta_{X^{2}} & 0 & 0 & C(\alpha) \\
0 & \beta_{X^{2}} & 0 & 0 & 0 & 0 & 0 \\
0 & 0 & C(\alpha) & 0 & 0 & 0 & 0 \\
\beta_{X^{2}} & 0 & 0 & \beta_{X^{4}} & 0 & 0 & \beta_{X^{2}}+\beta_{X^{4}} \\
0 & 0 & 0 & 0 & \beta_{X^{2}}+\beta_{X^{4}} & -\beta_{X^{2}}-\beta_{X^{4}} & 0 \\
0 & 0 & 0 & 0 & -\beta_{X^{2}}-\beta_{X^{4}} & \beta_{X^{2}}+\beta_{X^{4}} & 0 \\
C(\alpha) & 0 & 0 & \beta_{X^{2}}+\beta_{X^{4}} & 0 & 0 & D(\alpha)
\end{array}\right)
$$

where

$$
C(\alpha)=1+\beta_{X^{2}}-2 \alpha, \quad D(\alpha)=1+2 \beta_{X^{2}}+\beta_{X^{4}}-2 \alpha .
$$

We have that

$$
\operatorname{det}\left(\left.B(\alpha)\right|_{\left\{\mathbb{1}, \mathbb{X}, \mathbb{Y}, \mathbb{X}^{2}, \mathbb{X} \mathbb{Y}\right\}}\right)=\operatorname{det}\left(\left.B(\alpha)\right|_{\left\{1, \mathbb{X}^{2}\right\}}\right) \cdot \beta_{X^{2}} \cdot C(\alpha) \cdot\left(\beta_{X^{2}}+\beta_{X^{4}}\right),
$$

and

$$
\operatorname{det}\left(\left.B(\alpha)\right|_{\left\{\mathbb{1}, \mathbb{X}^{2}\right\}}\right)=\beta_{X^{4}}-\beta_{X^{2}}^{2}-2 \alpha \beta_{X^{4}} .
$$

Let $\alpha_{0}>0$ be the smallest positive number such that the rank of $B\left(\alpha_{0}\right)$ is smaller than 5. Not that $B\left(\alpha_{0}\right)$ is psd. By (6.36) and (6.37) we get that

$$
\alpha_{0}=\min \left(\frac{1+\beta_{X^{2}}}{2}, \frac{\beta_{X^{4}}-\beta_{X^{2}}^{2}}{2 \beta_{X^{4}}}\right)=\frac{1}{2}-\frac{\beta_{X^{2}}^{2}}{2 \beta_{X^{4}}} .
$$

The matrix $B\left(\alpha_{0}\right)$ is psd matrix of rank 4 and satisfies the relations

$$
\mathbb{X}^{2}=\frac{\beta_{X^{4}}}{\beta_{X^{2}}} \mathbb{1}, \quad \mathbb{X} \mathbb{Y}+\mathbb{Y} \mathbb{X}=\mathbf{0}, \quad \mathbb{Y}^{2}=\left(1+\frac{\beta_{X^{4}}}{\beta_{X^{2}}}\right) \mathbb{1} .
$$

By Theorem 3.1 it has a unique (up to orthogonal equivalence) 1 -atomic measure with an atom $(X, Y) \in\left(\mathbb{S R}^{2 \times 2}\right)^{2}$. Therefore $\mathcal{M}_{2}$ has a unique minimal measure of type $(2,1)$. Indeed, minimality follows by the following facts:

- Since $\mathcal{M}_{2}$ is a nc moment matrix, there must be at least one atom of size $>1$ in its representing measure.

- If there is exactly one atom of size 2 in the representing measure for $\mathcal{M}_{2}$, then there must be at least one atom of size 1 , since otherwise $\mathcal{M}_{2}$ would have rank at most 4 . Since $\beta_{X}=\beta_{Y}=0$, atoms $(0,1),(0,-1)$ occur in pairs with the same densities.

Claim 2: If $\beta$ admits a nc measure, then it has a representing measure with the atoms of size at most 2 .

Claim 2 follows by Proposition 6.1 and Theorem 6.8 (1).

Claim 3: If $\beta_{X} \neq 0$ or $\beta_{Y} \neq 0$, then $\beta$ admits a nc measure if and only if (6.35) holds.

Let us assume that $\beta_{X} \neq 0$ or $\beta_{Y} \neq 0$ and suppose that $\beta$ admits a nc measure. By Claim 2,

$$
\mathcal{M}_{2}=\sum_{i} \lambda_{i} \mathcal{M}_{2}^{\left(x_{i}, y_{i}\right)}+\sum_{j} \xi_{j} \mathcal{M}_{2}^{\left(X_{j}, Y_{j}\right)} .
$$

where $\left(x_{i}, y_{i}\right) \in \mathbb{R}^{2},\left(X_{j}, Y_{j}\right) \in \mathbb{S R}^{2 \times 2}, \lambda_{i}>0, \xi_{j}>0$ and $\sum_{i} \lambda_{i}+\sum_{j} \xi_{j}=1$. By Corollary 3.2,

$$
\beta_{X}^{(j)}=\beta_{Y}^{(j)}=\beta_{X^{3}}^{(j)}=\beta_{X^{2} Y}^{(j)}=\beta_{X Y^{2}}^{(j)}=\beta_{Y^{3}}^{(j)}=0,
$$


where $\beta_{w(X, Y)}^{(j)}$ are the moments of $\mathcal{M}_{2}^{\left(X_{j}, Y_{j}\right)}$. By the first paragraph in the proof of Theorem 6.11,

$$
\sum_{i} \lambda_{i} \mathcal{M}_{2}^{\left(x_{i}, y_{i}\right)}=\lambda_{+} \mathcal{M}_{2}^{(0,1)}+\lambda_{-} \mathcal{M}_{2}^{(0,-1)}
$$

where $\lambda_{ \pm} \geq 0$. Using (6.38), (6.39) and (6.40) we conclude that $\sum_{i} \lambda_{i} \mathcal{M}_{2}^{\left(x_{i}, y_{i}\right)}$ is of the form

$$
\sum_{i} \lambda_{i} \mathcal{M}_{2}^{\left(x_{i}, y_{i}\right)}=\left(\begin{array}{ccccccc}
a & 0 & \beta_{Y} & 0 & 0 & 0 & a \\
0 & 0 & 0 & 0 & 0 & 0 & 0 \\
\beta_{Y} & 0 & a & 0 & 0 & 0 & \beta_{Y} \\
0 & 0 & 0 & 0 & 0 & 0 & 0 \\
0 & 0 & 0 & 0 & 0 & 0 & 0 \\
0 & 0 & 0 & 0 & 0 & 0 & 0 \\
a & 0 & \beta_{Y} & 0 & 0 & 0 & a
\end{array}\right) \quad \text { for some } a \geq 0
$$

where

$$
\beta_{Y}=\lambda_{+}-\lambda_{-}, \quad a=\lambda_{+}+\lambda_{-} .
$$

Subclaim 3.1: If $\beta$ has a nc measure, then $\beta_{X}=0$.

Combining (6.38), (6.39) and (6.41), the subclaim follows.

Subclaim 3.2: We have that

$$
\sum_{i} \lambda_{i} \mathcal{M}_{2}^{\left(x_{i}, y_{i}\right)} \succeq\left|\beta_{Y}\right| \mathcal{M}_{2}^{\left(0, \operatorname{sign}\left(\beta_{Y}\right) 1\right)}=: A .
$$

where $\operatorname{sign}\left(\beta_{Y}\right)=+$ if $\beta_{Y} \geq 0$ and -1 otherwise.

Since $\beta_{Y}=\lambda_{+}-\lambda_{-}$and $\lambda_{ \pm} \geq 0$, it follows that $\lambda_{\operatorname{sign}\left(\beta_{Y}\right)} \geq\left|\operatorname{sign}\left(\beta_{Y}\right)\right|$. Thus, (6.42) follows.

$\mathcal{M}_{2}-A$ is of the form

$$
\left(\begin{array}{ccccccc}
1-\left|\beta_{Y}\right| & 0 & 0 & \beta_{X^{2}} & 0 & 0 & E \\
0 & \beta_{X^{2}} & 0 & 0 & 0 & 0 & 0 \\
0 & 0 & E & 0 & 0 & 0 & 0 \\
\beta_{X^{2}} & 0 & 0 & \beta_{X^{4}} & 0 & 0 & \beta_{X^{2}}+\beta_{X^{4}} \\
0 & 0 & 0 & 0 & \beta_{X^{2}}+\beta_{X^{4}} & -\beta_{X^{2}}-\beta_{X^{4}} & 0 \\
0 & 0 & 0 & 0 & -\beta_{X^{2}}-\beta_{X^{4}} & \beta_{X^{2}}+\beta_{X^{4}} & 0 \\
E & 0 & 0 & \beta_{X^{2}}+\beta_{X^{4}} & 0 & 0
\end{array}\right),
$$

where

$$
E=1+\beta_{X^{2}}-\left|\beta_{Y}\right|, \quad F=E+\beta_{X^{2}}+\beta_{X^{4}} .
$$

By Subclaim 3.1, $\mathcal{M}_{2}-\sum_{i} \lambda_{i} \mathcal{M}_{2}^{\left(x_{i}, y_{i}\right)} \preceq \mathcal{M}_{2}-A$. Necessary conditions for the existence of a measure by Proposition 2.1 and Corollary 2.3 for a nc moment matrix $\mathcal{M}_{2}-A$ are that $\mathcal{M}_{2}-A$ is psd and $\left.\left(\mathcal{M}_{2}-A\right)\right|_{\{\mathbb{1}, \mathbb{X}, \mathbb{Y}, \mathbb{X} \mathbb{Y}\}}$ is pd. The latter is equivalent to

(6.43) $1-\left|\beta_{Y}\right|>0, \quad \beta_{X^{2}}>0, \quad 1+\beta_{X^{2}}-\left|\beta_{Y}\right|>0, \quad \beta_{X^{2}}+\beta_{X^{4}}>0, \quad \beta_{X^{4}} \geq 0, \quad \operatorname{det}\left(\left.\mathcal{M}_{2}\right|_{\left\{\mathbb{1}, \mathbb{X}^{2}\right\}}\right) \geq 0$

Further on, using Subclaim 3.1 this system is equivalent to the conditions (6.35). This proves Claim 3.

Claim 4: Minimal measures are as stated in the theorem.

If $\beta_{X}=\beta_{Y}=0$, Claim 4 follows by Claim 1. Suppose $\beta_{Y} \neq 0$. Let $A$ be as in the proof of Claim 3. The following statements are true:

(1) Minimal measure is unique of type $(1,1)$ if and only if the rank of $\mathcal{M}_{2}-A \succeq 0$ is 4 .

(2) Minimal measure is unique of type $(2,1)$ if and only if the rank of $\mathcal{M}_{2}-A \succeq 0$ is 5 in which case we subtract $\alpha\left(\mathcal{M}_{2}^{(0,1)}+\mathcal{M}_{2}^{(0,-1)}\right)$ with the smallest $\alpha>0$ such that the rank falls to 4 .

The rank of $\mathcal{M}_{2}-A$ is 5 exactly when in (6.43) we have $\operatorname{det}\left(\left.\mathcal{M}_{2}\right|_{\left\{\mathbb{1}, \mathbb{X}^{2}\right\}}\right)>0$ which is exactly when in addition $\beta_{X^{4}}>\frac{\beta_{X^{2}}^{2}}{1-\left|\beta_{Y}\right|}$. 
6.4. Pair $\mathbb{X} \mathbb{Y}+\mathbb{Y} \mathbb{X}=\mathbf{0}$ and $\mathbb{Y}^{2}=\mathbb{X}^{2}$. In this subsection we study a nc sequence $\beta$ with a moment matrix $\mathcal{M}_{2}$ of rank 5 satisfying the relations $\mathbb{X} \mathbb{Y}+\mathbb{Y} \mathbb{X}=\mathbf{0}$ and $\mathbb{Y}^{2}+\mathbb{X}^{2}=\mathbb{1}$. In Theorem 6.14 we characterize exactly when $\beta$ admits a nc measure. Moreover, we classify the type and uniqueness of the minimal measure.

The form of $\mathcal{M}_{2}$ is given by the following proposition.

Proposition 6.12. Suppose $\beta \equiv \beta^{(4)}$ is a nc sequence with a moment matrix $\mathcal{M}_{2}$ satisfying the relations

$$
\mathbb{X} \mathbb{Y}+\mathbb{Y}=\mathbf{0} \text { and } \mathbb{Y}^{2}=\mathbb{X}^{2} .
$$

Then $\mathcal{M}_{2}$ is of the form

$$
\left(\begin{array}{ccccccc}
\beta_{1} & \beta_{X} & \beta_{Y} & \beta_{X^{2}} & 0 & 0 & \beta_{X^{2}} \\
\beta_{X} & \beta_{X^{2}} & 0 & 0 & 0 & 0 & 0 \\
\beta_{Y} & 0 & \beta_{X^{2}} & 0 & 0 & 0 & 0 \\
\beta_{X^{2}} & 0 & 0 & \beta_{X^{4}} & 0 & 0 & \beta_{X^{4}} \\
0 & 0 & 0 & 0 & \beta_{X^{4}} & -\beta_{X^{4}} & 0 \\
0 & 0 & 0 & 0 & -\beta_{X^{4}} & \beta_{X^{4}} & 0 \\
\beta_{X^{2}} & 0 & 0 & \beta_{X^{4}} & 0 & 0 & \beta_{X^{4}}
\end{array}\right) .
$$

Proof. The relations (6.44) give us the following system in $\mathcal{M}_{2}$

$$
\begin{array}{rrr}
2 \beta_{X Y}=0, & \beta_{X^{2} Y^{2}}+\beta_{X Y X Y}=0, & \beta_{Y^{3}}=\beta_{X^{2} Y}, \\
2 \beta_{X^{2} Y}=0, & 2 \beta_{X Y^{3}}=0, & \beta_{X^{2} Y^{2}}=\beta_{X^{4}}, \\
2 \beta_{X Y^{2}}=0, & \beta_{Y^{2}}=\beta_{X^{2}}, & \beta_{X Y^{3}}=\beta_{X^{3} Y}, \\
2 \beta_{X^{3} Y}=0, & \beta_{X Y^{2}}=\beta_{X^{3}}, & \beta_{Y^{4}}=\beta_{X^{2} Y^{2}} .
\end{array}
$$

The solution to (6.46) is given by

$$
\begin{array}{r}
\beta_{X Y}=\beta_{X^{3}}=\beta_{X^{2} Y}=\beta_{X Y^{2}}=\beta_{Y^{3}}=\beta_{X^{3} Y}=\beta_{X Y^{3}}=0, \\
\beta_{Y^{2}}=\beta_{X^{2}}, \\
\beta_{X Y X Y}=-\beta_{X^{2} Y^{2}}=-\beta_{Y^{4}}=-\beta_{X^{4}},
\end{array}
$$

and thus $\mathcal{M}_{2}$ takes the form (6.45).

Proposition 6.13. Suppose $\beta \equiv \beta^{(4)}$ is a nc sequence with a moment matrix $\mathcal{M}_{2}$ of rank 5 satisfying the relations $\mathbb{X} \mathbb{Y}+\mathbb{Y} \mathbb{X}=\mathbf{0}$ and $\mathbb{Y}^{2}=\mathbb{X}^{2}$. Then $\mathcal{M}_{2}$ is positive semidefinite if and only if

$$
0<\beta_{X^{2}}, \quad\left|\beta_{X}\right|<\sqrt{\beta_{X^{2}}}, \quad\left|\beta_{Y}\right|<\sqrt{\beta_{X^{2}}-\beta_{X}^{2}}, \quad \frac{\beta_{X^{2}}^{3}}{\beta_{X^{2}}-\beta_{Y}^{2}-\beta_{X}^{2}}<\beta_{X^{4}} .
$$

Proof. Write $\mathcal{M}_{2}$ as $\mathcal{M}_{2}=\left(\begin{array}{cc}A & B \\ B^{t} & C\end{array}\right)$ where

$$
A=\left.\mathcal{M}_{2}\right|_{\left\{1, \mathbb{X}, \mathbb{Y}, \mathbb{X}^{2}, \mathbb{X} \mathbb{Y}\right\}}, \quad B=\left.\mathcal{M}_{2}\right|_{\left\{\mathbb{1}, \mathbb{X}, \mathbb{Y}, \mathbb{X}^{2}, \mathbb{X} \mathbb{Y}\right\},\left\{\mathbb{Y X}, \mathbb{Y}^{2}\right\}} \quad C=\left.\mathcal{M}_{2}\right|_{\left\{\mathbb{Y X}, \mathbb{Y}^{2}\right\}} .
$$

Since $\mathcal{M}_{2}$ satisfies the relations $\mathbb{X} \mathbb{Y}+\mathbb{Y} \mathbb{X}=\mathbf{0}$ and $\mathbb{X}^{2}=\mathbb{Y}^{2}$, it follows that $\operatorname{rank}\left(\mathcal{M}_{2}\right)=\operatorname{rank}(A)=5$. Now by Lemma 6.4, $\mathcal{M}_{2}$ is psd if and only if $A$ is psd. Since $\operatorname{rank}(A)=5, A$ is psd if and only if $A$ is positive definite (pd). By Sylvester's criterion $A$ is pd if and only if all its principal minors are positive. The principal minors $\alpha_{i}, i=1, \ldots, 5$, of $A$ are

$$
\alpha_{1}=1, \quad \alpha_{2}=\beta_{X^{2}}-\beta_{X}^{2}, \quad \alpha_{3}=-\beta_{Y}^{2} \beta_{X^{2}}-\beta_{X}^{2} \beta_{X^{2}}+\beta_{X^{2}}^{2}, \quad \alpha_{4}=-\beta_{X^{2}}^{4}+\beta_{X^{4}} \alpha_{3}, \quad \alpha_{5}=\alpha_{4} \beta_{X^{4}} .
$$

Thus

$$
\alpha_{2}>0 \Leftrightarrow 0 \leq\left|\beta_{X}\right|<\sqrt{\beta_{X^{2}}}, \quad \alpha_{3}>0 \Leftrightarrow\left|\beta_{Y}\right|<\sqrt{\beta_{X^{2}}-\beta_{X}^{2}}, \quad \alpha_{4}>0 \Leftrightarrow \frac{\beta_{X^{2}}^{3}}{\beta_{X^{2}}-\beta_{Y}^{2}-\beta_{X}^{2}}<\beta_{X^{4}} .
$$

Note that $\alpha_{2}, \alpha_{3}, \alpha_{4}>0$ also imply $\alpha_{5}>0$ (since $\beta_{X^{4}}>0$ if $\left.A\right|_{\left\{\mathbb{1}, \mathbb{X}, \mathbb{Y}, \mathbb{X}^{2}\right\}}$ is pd). This proves the proposition.

The following theorem characterizes normalized nc sequences $\beta$ with a moment matrix $\mathcal{M}_{2}$ of rank 5 satisfying the relations $\mathbb{X} \mathbb{Y}+\mathbb{Y} \mathbb{X}=\mathbf{0}$ and $\mathbb{X}^{2}=\mathbb{Y}^{2}$, which admit a nc measure.

Theorem 6.14. Suppose $\beta \equiv \beta^{(4)}$ is a nc sequence with a moment matrix $\mathcal{M}_{2}$ of rank 5 satisfying the relations $\mathbb{X} \mathbb{Y}+\mathbb{Y} \mathbb{X}=\mathbf{0}$ and $\mathbb{X}^{2}=\mathbb{Y}^{2}$. Then $\beta$ admits a nc measure if and only if $\mathcal{M}_{2}$ is positive semidefinite and $\beta_{X}=\beta_{Y}=0$. The minimal measure is unique (up to orthogonal equivalence) and of type $(1,1)$. 
Proof. First note that the only pair $(x, y) \in \mathbb{R}^{2}$ satisfying the equations $x y+y x=0$ and $y^{2}=x^{2}$ is

$$
(0,0) \in \mathbb{R}^{2} .
$$

By Lemma 2.2 this is the only pair from $\mathbb{R}^{2}$ which can be an atom of size 1 in the nc measure of $\beta$. We have

$$
\mathcal{M}_{2}^{(0,0)}=(1) \oplus \mathbf{0}_{6},
$$

where $\mathbf{0}_{6}$ stands for the $6 \times 6$ matrix with only zero entries.

Claim 1: If $\beta_{X}=\beta_{Y}=0$, then $\beta$ admits a nc measure. Moreover, the minimal measure is unique (up to orthogonal equivalence) and of type $(1,1)$.

Using (6.45) we see that $\mathcal{M}_{2}$ is of the form

$$
\mathcal{M}_{2}=\left(\begin{array}{ccccccc}
1 & 0 & 0 & \beta_{X^{2}} & 0 & 0 & \beta_{X^{2}} \\
0 & \beta_{X^{2}} & 0 & 0 & 0 & 0 & 0 \\
0 & 0 & \beta_{X^{2}} & 0 & 0 & 0 & 0 \\
\beta_{X^{2}} & 0 & 0 & \beta_{X^{4}} & 0 & 0 & \beta_{X^{4}} \\
0 & 0 & 0 & 0 & \beta_{X^{4}} & -\beta_{X^{4}} & 0 \\
0 & 0 & 0 & 0 & -\beta_{X^{4}} & \beta_{X^{4}} & 0 \\
\beta_{X^{2}} & 0 & 0 & \beta_{X^{4}} & 0 & 0 & \beta_{X^{4}}
\end{array}\right) .
$$

We define the matrix function

$$
B(\alpha):=\mathcal{M}_{2}-\alpha \mathcal{M}_{2}^{(0,0)}
$$

such that

$$
B(\alpha)=\left(\begin{array}{ccccccc}
1-\alpha & 0 & 0 & \beta_{X^{2}} & 0 & 0 & \beta_{X^{2}} \\
0 & \beta_{X^{2}} & 0 & 0 & 0 & 0 & 0 \\
0 & 0 & \beta_{X^{2}} & 0 & 0 & 0 & 0 \\
\beta_{X^{2}} & 0 & 0 & \beta_{X^{4}} & 0 & 0 & \beta_{X^{4}} \\
0 & 0 & 0 & 0 & \beta_{X^{4}} & -\beta_{X^{4}} & 0 \\
0 & 0 & 0 & 0 & -\beta_{X^{4}} & \beta_{X^{4}} & 0 \\
\beta_{X^{2}} & 0 & 0 & \beta_{X^{4}} & 0 & 0 & \beta_{X^{4}}
\end{array}\right) .
$$

We have that

$$
\operatorname{det}\left(\left.B(\alpha)\right|_{\left\{\mathbb{1}, \mathbb{X}, \mathbb{Y}, \mathbb{X}^{2}, \mathbb{X} \mathbb{Y}\right\}}\right)=\operatorname{det}\left(\left.B(\alpha)\right|_{\left\{\mathbb{1}, \mathbb{X}^{2}\right\}}\right) \cdot \beta_{X^{2}}^{2} \cdot \beta_{X^{4}},
$$

and

$$
\operatorname{det}\left(\left.B(\alpha)\right|_{\left\{\mathbb{1}, \mathbb{X}^{2}\right\}}\right)=\beta_{X^{4}}-\beta_{X^{2}}^{2}-\alpha \beta_{X^{4}} .
$$

Let $\alpha_{0}>0$ be the smallest positive number such that the rank of $B\left(\alpha_{0}\right)$ is smaller than 5. By (6.51) and (6.52) we get that

$$
\alpha_{0}=\frac{\beta_{X^{4}}-\beta_{X^{2}}^{2}}{\beta_{X^{4}}} .
$$

The matrix $B\left(\alpha_{0}\right)$ is psd matrix of rank 4 and satisfies the relations

$$
\mathbb{X}^{2}=\frac{\beta_{X^{4}}}{\beta_{X^{2}}} \mathbb{1}, \quad \mathbb{X} \mathbb{Y}+\mathbb{Y} \mathbb{X}=\mathbf{0}, \quad \mathbb{Y}^{2}=\frac{\beta_{X^{4}}}{\beta_{X^{2}}} \mathbb{1} .
$$

By Theorem 3.1 it has a unique (up to orthogonal equivalence) 1 -atomic measure with an atom $(X, Y) \in\left(\mathbb{S R}^{2 \times 2}\right)^{2}$. Therefore $\mathcal{M}_{2}$ has a unique minimal measure of type $(1,1)$. Indeed, minimality follows by the following facts:

- Since $\mathcal{M}_{2}$ is a nc moment matrix, there must be at least one atom of size $>1$ in its representing measure.

- If there is exactly one atom of size 2 in the representing measure for $\mathcal{M}_{2}$, then there must be at least one atom of size 1 , since otherwise $\mathcal{M}_{2}$ would have rank at most 4 .

Claim 2: If $\beta_{X} \neq 0$ or $\beta_{Y} \neq 0$, then $\beta$ does not admit a nc measure.

Subclaim 2.1: If $\beta$ admits a nc measure, then it has a representing measure with the atoms of size at most 2 .

Subclaim 2.1 follows by Proposition 6.1 and Claim 1. 
Suppose that $\beta$ admits a nc measure and $\beta_{X} \neq 0$ or $\beta_{Y} \neq 0$. By Subclaim 2.1,

$$
\mathcal{M}_{2}=\sum_{i} \lambda_{i} \mathcal{M}_{2}^{\left(x_{i}, y_{i}\right)}+\sum_{j} \xi_{j} \mathcal{M}_{2}^{\left(X_{j}, Y_{j}\right)}
$$

where $\left(x_{i}, y_{i}\right) \in \mathbb{R}^{2},\left(X_{j}, Y_{j}\right) \in \mathbb{S R}^{2 \times 2}, \lambda_{i}>0, \xi_{j}>0$ and $\sum_{i} \lambda_{i}+\sum_{j} \xi_{j}=1$. By Corollary 3.2,

$$
\beta_{X}^{(j)}=\beta_{Y}^{(j)}=\beta_{X^{3}}^{(j)}=\beta_{X^{2} Y}^{(j)}=\beta_{X Y^{2}}^{(j)}=\beta_{Y^{3}}^{(j)}=0,
$$

where $\beta_{w(X, Y)}^{(j)}$ are the moments of $\mathcal{M}_{2}^{\left(X_{j}, Y_{j}\right)}$. By the first paragraph in the proof of Theorem 6.14,

$$
\sum_{i} \lambda_{i} \mathcal{M}_{2}^{\left(x_{i}, y_{i}\right)}=\lambda \mathcal{M}_{2}^{(0,0)}
$$

where $\lambda>0$. Using (6.53), (6.54) and (6.55) it follows that

$$
0=\sum_{j} \beta_{X}^{(j)}=\beta_{X} \quad \text { and } \quad 0=\sum_{j} \beta_{Y}^{(j)}=\beta_{Y}
$$

This is a contradiction with the assumption $\beta_{X} \neq 0$ or $\beta_{Y} \neq 0$, which proves Claim 2.

\section{BQTMP With $\mathcal{M}_{2}$ IN THE BASIC CASES 1 AND 2 OF RANK 6}

In this section we solve the BQTMP for $\mathcal{M}_{2}$ in the basic cases 1 and 2 of rank 6 given by Proposition 4.1. In Subsections 7.1 and 7.2 we study each case separately. We characterize when $\mathcal{M}_{2}$ admits a nc measure, see Theorems 7.5 and 7.8. Corollaries 7.6 and 7.9 translate the existence of a nc measure into the feasibility problem of three linear matrix inequalities and a rank-to-variety condition from Theorem 2.7.

The following proposition states that if $\beta$ has a moment matrix $\mathcal{M}_{2}$ of rank 6 in the basic cases 1,2 or 3 given by Proposition 4.1 (2) and $\beta$ admits a nc measure, then it has a representing measure with the atoms of size at most 2.

Proposition 7.1. Let us fix a basic case relation 1, 2 or 3 given by Proposition 4.1 (2) and denote it by $R$. If a nc sequence $\beta$ with a moment matrix $\mathcal{M}_{2}(\beta)$ of rank 6 satisfying $R$ admits a nc measure, then it admits a nc measure with atoms of size at most 2.

Proof. Suppose $\beta$ has a moment matrix $\mathcal{M}_{2}(\beta)$ of rank 6 satisfying $R$ and admits a nc measure. By Proposition 5.1 we may assume that all the atoms $\left(X_{i}, Y_{i}\right) \in\left(\mathbb{S R}^{u_{i} \times u_{i}}\right)^{2}$ of size $u_{i}>1$ are of the form

$$
X_{i}=\left(\begin{array}{cc}
\gamma_{i} I_{t_{i}} & B_{i} \\
B_{i}^{t} & -\gamma_{i} I_{t_{i}}
\end{array}\right), \quad Y_{i}=\left(\begin{array}{cc}
\mu_{i} I_{t_{i}} & \mathbf{0} \\
\mathbf{0} & -\mu_{i} I_{t_{i}}
\end{array}\right),
$$

where $\gamma_{i}>0, \mu_{i}>0$ and $B_{i}$ are $t_{i} \times t_{i}$ matrices. Calculating $Y_{i}^{2}$ we get

$$
Y_{i}^{2}=\mu_{i}^{2} I_{2 t_{i}} .
$$

Therefore $\mathcal{M}_{2}^{\left(X_{i}, Y_{i}\right)}$ satisfies the relations $R$ and (7.1) and hence it is of rank at most 5. By the results from Sections ?? and 6 it can be represented by the atoms of size at most 2 .

The following two propositions say more about the minimal measure.

Proposition 7.2. Let us fix a basic case relation 1, 2 or 3 given by Proposition 4.1 (2) and denote it by $R$. If a sequence $\beta$ with a moment matrix $\mathcal{M}_{2}$ satisfying $R$ admits a nc measure of type $(k, 1)$, then

(1) $2 \leq k \leq 5$ if $R$ is equal to $\mathbb{Y}^{2}=\mathbb{1}-\mathbb{X}^{2}$ or $\mathbb{Y}^{2}=\mathbb{1}+\mathbb{X}^{2}$.

(2) $2 \leq k \leq 6$ if $R$ is equal to $\mathbb{X} \mathbb{Y}+\mathbb{Y} \mathbb{X}=\mathbf{0}$.

Proof. By assumption,

$$
\mathcal{M}_{2}=\sum_{i=1}^{k} \lambda_{i} \mathcal{M}_{2}^{\left(x_{i}, y_{i}\right)}+\xi \mathcal{M}_{2}^{(X, Y)},
$$

where $\left(x_{i}, y_{i}\right) \in \mathbb{R}^{2},(X, Y) \in\left(\mathbb{S R}^{2 \times 2}\right)^{2}, k \in \mathbb{N}, \lambda_{i}>0, \xi>0$ and $\sum_{i=1}^{k} \lambda_{i}+\xi=1$. Equivalently

$$
\mathcal{M}_{2}-\xi \mathcal{M}_{2}^{(X, Y)}=\sum_{i=1}^{k} \lambda_{i} \mathcal{M}_{2}^{\left(x_{i}, y_{i}\right)}
$$

Since $\sum_{i=1}^{k} \lambda_{i} \mathcal{M}_{2}^{\left(x_{i}, y_{i}\right)}$ is a cm moment matrix of rank at most 6 satisfying the relation $R$, then by Theorem 2.7 
(1) $2 \leq k \leq 5$ if $R$ is equal to $\mathbb{Y}^{2}=\mathbb{1}-\mathbb{X}^{2}$ or $\mathbb{Y}^{2}=\mathbb{1}+\mathbb{X}^{2}$,

(2) $2 \leq k \leq 6$ if $R$ is equal to $\mathbb{X} \mathbb{Y}+\mathbb{Y} \mathbb{X}=\mathbf{0}$,

which proves Proposition 7.2.

Proposition 7.3. Let us fix a basic case relation 1 or 2 given by Proposition 4.1 (2) and denote it by R. If every sequence $\beta$ with $\beta_{X}=\beta_{Y}=\beta_{X^{3}}=\beta_{X^{2} Y}=\beta_{Y}^{3}=0$ and a moment matrix $\mathcal{M}_{2}(\beta)$ of rank 6 with column relation $R$, admits a nc measure with exactly one atom of size 2 and some atoms of size 1 , then every sequence $\widetilde{\beta}$ which admits a nc measure and has a moment matrix $\widetilde{\mathcal{M}}_{2}$ of rank 6 with the column relation $R$, admits a nc measure with exactly one atom of size 2 and some atoms of size 1.

Proof. Suppose $\widetilde{\beta}$ admits a nc measure and has a moment matrix $\widetilde{\mathcal{M}}_{2}$ of rank 6 with column relation $R$. By Proposition 5.1 we may assume that all the atoms $\left(X_{i}, Y_{i}\right) \in\left(\mathbb{S R}^{u_{i} \times u_{i}}\right)^{2}$ of size $u_{i}>1$ are of the form

$$
X_{i}=\left(\begin{array}{cc}
\gamma_{i} I_{t_{i}} & B_{i} \\
B_{i}^{t} & -\gamma_{i} I_{t_{i}}
\end{array}\right), \quad Y_{i}=\left(\begin{array}{cc}
\mu_{i} I_{t_{i}} & \mathbf{0} \\
\mathbf{0} & -\mu_{i} I_{t_{i}}
\end{array}\right),
$$

where $\gamma_{i} \geq 0, \mu_{i}>0$ and $B_{i}$ are $t_{i} \times t_{i}$ matrices. Calculating $X_{i}^{3}, X_{i}^{2} Y_{i}$ and $Y_{i}^{3}$ we get

$$
\begin{aligned}
X_{i}^{3} & =\left(\begin{array}{cc}
\gamma_{i}\left(\gamma_{i}^{2} I_{t_{i}}+B_{i} B_{i}^{t}\right) & \left(\gamma_{i}^{2} I_{t_{i}}+B_{i} B_{i}^{t}\right) B_{i} \\
\left(\gamma_{i}^{2} I_{t_{i}}+B_{i}^{t} B_{i}\right) B_{i}^{t} & -\gamma_{i}\left(\gamma_{i}^{2} I_{t_{i}}+B_{i}^{t} B_{i}\right)
\end{array}\right), \\
X_{i}^{2} Y_{i} & =\left(\begin{array}{cc}
\mu_{i}\left(\gamma_{i}^{2} I_{t_{i}}+B_{i} B_{i}^{t}\right) & 0 \\
0 & -\mu_{i}\left(\gamma_{i}^{2} I_{t_{i}}+B_{i}^{t} B_{i}\right)
\end{array}\right), \\
Y_{i}^{3} & =\left(\begin{array}{cc}
\mu_{i}^{3} I_{t_{i}} & 0 \\
0 & -\mu_{i}^{3} I_{t_{i}}
\end{array}\right) .
\end{aligned}
$$

Therefore $\sum_{i} \mathcal{M}_{2}^{\left(X_{i}, Y_{i}\right)}$ satisfies $\beta_{X}=\beta_{Y}=\beta_{X^{3}}=\beta_{X^{2} Y}=\beta_{Y^{3}}=0$. If the rank of $\sum_{i} \mathcal{M}_{2}^{\left(X_{i}, Y_{i}\right)}$ is at most 5, then $\sum_{i} \mathcal{M}_{2}^{\left(X_{i}, Y_{i}\right)}$ can be represented by exactly one atom of size 2 and some atoms of size 1 by the results of previous sections. Else the rank of $\sum_{i} \mathcal{M}_{2}^{\left(X_{i}, Y_{i}\right)}$ is 6 and the same conclusion follows by assumption.

7.1. Relation $\mathbb{Y}^{2}=\mathbb{1}-\mathbb{X}^{2}$. In this subsection we study a nc sequence $\beta$ with a moment matrix $\mathcal{M}_{2}$ of rank 6 satisfying the relation $\mathbb{Y}^{2}=\mathbb{1}-\mathbb{X}^{2}$. In Theorem 7.5 we characterize when $\beta$ admits a nc measure. In Corollary 7.6 we show that the existence of a nc measure is equivalent to the feasibility problem of three linear matrix inequalities (LMIs) and rank-to-cardinality condition from Theorem 2.7.

The form of $\mathcal{M}_{2}$ is given by the following proposition.

Proposition 7.4. Let $\beta \equiv \beta^{(4)}$ be a nc sequence with a moment matrix $\mathcal{M}_{2}$ satisfying the relation

$$
\mathbb{Y}^{2}=\mathbb{1}-\mathbb{X}^{2}
$$

Then $\mathcal{M}_{2}$ is of the form

$$
\left(\begin{array}{ccccccc}
\beta_{1} & \beta_{X} & \beta_{Y} & \beta_{X^{2}} & \beta_{X Y} & \beta_{X Y} & \beta_{1}-\beta_{X^{2}} \\
\beta_{X} & \beta_{X^{2}} & \beta_{X Y} & \beta_{X^{3}} & \beta_{X^{2} Y} & \beta_{X^{2} Y} & \beta_{X}-\beta_{X^{3}} \\
\beta_{Y} & \beta_{X Y} & \beta_{1}-\beta_{X^{2}} & \beta_{X^{2} Y} & \beta_{X}-\beta_{X^{3}} & \beta_{X}-\beta_{X^{3}} & \beta_{Y}-\beta_{X^{2} Y} \\
\beta_{X^{2}} & \beta_{X^{3}} & \beta_{X^{2} Y} & \beta_{X^{4}} & \beta_{X^{3} Y} & \beta_{X^{3} Y} & \beta_{X^{2}}-\beta_{X^{4}} \\
\beta_{X Y} & \beta_{X^{2} Y} & \beta_{X}-\beta_{X^{3}} & \beta_{X^{3} Y} & \beta_{X^{2}}-\beta_{X^{4}} & \beta_{X Y X Y} & \beta_{X Y}-\beta_{X^{3} Y} \\
\beta_{X Y} & \beta_{X^{2} Y} & \beta_{X}-\beta_{X^{3}} & \beta_{X^{3} Y} & \beta_{X Y X} & \beta_{X^{2}}-\beta_{X^{4}} & \beta_{X Y}-\beta_{X^{3} Y} \\
\beta_{1}-\beta_{X^{2}} & \beta_{X}-\beta_{X^{3}} & \beta_{Y}-\beta_{X^{2} Y} & \beta_{X^{2}}-\beta_{X^{4}} & \beta_{X Y}-\beta_{X^{3} Y} & \beta_{X Y}-\beta_{X^{3} Y} & \beta_{1}-2 \beta_{X^{2}}+\beta_{X^{4}}
\end{array}\right) .
$$

Proof. The relation (7.4) gives us the following system in $\mathcal{M}_{2}$

$$
\begin{array}{rr}
\beta_{Y^{2}}=\beta_{1}-\beta_{X^{2}}, & \beta_{X^{2} Y^{2}}=\beta_{X^{2}}-\beta_{X^{4}}, \\
\beta_{X Y^{2}}=\beta_{X}-\beta_{X^{3}}, & \beta_{X Y^{3}}=\beta_{X Y}-\beta_{X^{3} Y}, \\
\beta_{Y^{3}}=\beta_{Y}-\beta_{X^{2} Y}, & \beta_{Y^{4}}=\beta_{Y^{2}}-\beta_{X^{2} Y^{2}} .
\end{array}
$$

Plugging in the expressions for $\beta_{Y^{2}}$ and $\beta_{X^{2} Y^{2}}$ in the expression for $\beta_{Y^{4}}$ gives the form (7.5) of $\mathcal{M}_{2}$.

The following theorem characterizes normalized nc sequences $\beta$ with a moment matrix $\mathcal{M}_{2}$ of rank 6 satisfying the relation $\mathbb{Y}^{2}=\mathbb{1}-\mathbb{X}^{2}$, which admit a nc measure. 
Theorem 7.5. Suppose $\beta \equiv \beta^{(4)}$ is a normalized nc sequence with a moment matrix $\mathcal{M}_{2}$ of rank 6 satisfying the relation $\mathbb{Y}^{2}=\mathbb{1}-\mathbb{X}^{2}$. Then $\beta$ admits a nc measure if and only if $\mathcal{M}_{2}$ is positive semidefinite and one of the following is true:

(1) $\beta_{X}=\beta_{Y}=\beta_{X^{3}}=\beta_{X^{2} Y}=0$. Moreover, there exists a nc measure of type $(4,1)$.

(2) There exist

$$
a_{1} \in(0,1), \quad a_{2} \in\left(-2 \sqrt{a_{1}\left(1-a_{1}\right)}, 2 \sqrt{a_{1}\left(1-a_{1}\right)}\right)
$$

such that

$$
M:=\mathcal{M}_{2}-\xi \mathcal{M}_{2}^{(X, Y)}
$$

is a positive semidefinite cm moment matrix satisfying $\operatorname{rank} M \leq \operatorname{card} \mathcal{V}_{M}$, where $\mathcal{V}_{M}$ is the variety associated to $M$ (as in Theorem 2.7),

$$
\begin{aligned}
X=\left(\begin{array}{cc}
\sqrt{a_{1}} & 0 \\
0 & -\sqrt{a_{1}}
\end{array}\right), \quad Y & =\sqrt{\left(1-a_{1}\right)}\left(\begin{array}{cc}
\frac{a}{2} & \frac{1}{2} \sqrt{4-a^{2}} \\
\frac{1}{2} \sqrt{4-a^{2}} & -\frac{a}{2}
\end{array}\right), \\
a & =\frac{a_{2}}{\sqrt{a_{1}\left(1-a_{1}\right)}},
\end{aligned}
$$

and $\xi>0$ is the smallest positive number such that the rank of $\mathcal{M}_{2}-\xi \mathcal{M}_{2}^{(X, Y)}$ is smaller than the rank of $\mathcal{M}_{2}$.

Proof. First we will prove (1). $\mathcal{M}_{2}$ is of the form

$$
\left(\begin{array}{ccccccc}
1 & 0 & 0 & \beta_{X^{2}} & \beta_{X Y} & \beta_{X Y} & 1-\beta_{X^{2}} \\
0 & \beta_{X^{2}} & \beta_{X Y} & 0 & 0 & 0 & 0 \\
0 & \beta_{X Y} & 1-\beta_{X^{2}} & 0 & 0 & 0 & 0 \\
\beta_{X^{2}} & 0 & 0 & \beta_{X^{4}} & \beta_{X^{3} Y} & \beta_{X^{3} Y} & \beta_{X^{2}}-\beta_{X^{4}} \\
\beta_{X Y} & 0 & 0 & \beta_{X^{3} Y} & \beta_{X^{2}}-\beta_{X^{4}} & \beta_{X Y X Y} & \beta_{X Y}-\beta_{X^{3} Y} \\
\beta_{X Y} & 0 & 0 & \beta_{X^{3} Y} & \beta_{X Y X Y} & \beta_{X^{2}}-\beta_{X^{4}} & \beta_{X Y}-\beta_{X^{3} Y} \\
1-\beta_{X^{2}} & 0 & 0 & \beta_{X^{2}}-\beta_{X^{4}} & \beta_{X Y}-\beta_{X^{3} Y} & \beta_{X Y}-\beta_{X^{3} Y} & 1-2 \beta_{X^{2}}+\beta_{X^{4}}
\end{array}\right) .
$$

We define the matrix function

$$
B(\alpha):=\mathcal{M}_{2}-\alpha\left(\mathcal{M}_{2}^{(1,0)}+\mathcal{M}_{2}^{(-1,0)}\right)
$$

We have that

$$
B(\alpha)=\left(\begin{array}{ccccccc}
1-2 \alpha & 0 & 0 & \beta_{X^{2}}-2 \alpha & \beta_{X Y} & \beta_{X Y} & D \\
0 & \beta_{X^{2}}-\alpha & \beta_{X Y} & 0 & 0 & 0 & 0 \\
0 & \beta_{X Y} & D & 0 & 0 & 0 & 0 \\
\beta_{X^{2}}-2 \alpha & 0 & 0 & \beta_{X^{4}}-2 \alpha & \beta_{X^{3} Y} & \beta_{X^{3} Y} & C \\
\beta_{X Y} & 0 & 0 & \beta_{X^{3} Y} & C & E & \beta_{X Y}-\beta_{X^{3} Y} \\
\beta_{X Y} & 0 & 0 & \beta_{X^{3} Y} & E & C & \beta_{X Y}-\beta_{X^{3} Y} \\
D & 0 & 0 & C & \beta_{X Y}-\beta_{X^{3} Y} & \beta_{X Y}-\beta_{X^{3} Y} & D-C
\end{array}\right)
$$

where

$$
C=\beta_{X^{2}}-\beta_{X^{4}}, \quad D=1-\beta_{X^{2}}, \quad E=\beta_{X Y X Y}
$$

We have that

(7.8) $\operatorname{det}\left(\left.B(\alpha)\right|_{\left\{\mathbb{1}, \mathbb{X}, \mathbb{Y}, \mathbb{X}^{2}, \mathbb{X Y}, \mathbb{Y X}\right\}}\right)=-\left(\beta_{X Y X Y}-\beta_{X^{2}}+\beta_{X^{4}}\right)\left(-\beta_{X Y}^{2}+\beta_{X^{2}}-\beta_{X^{2}}^{2}+2 \alpha\left(-1+\beta_{X}^{2}\right)\right)(-F+2 \alpha G)$, where

$$
\begin{aligned}
& F=\beta_{X Y X Y}\left(\beta_{X^{2}}^{2}-\beta_{X^{4}}\right)+\beta_{X^{2}}\left(\beta_{X^{2}}^{2}-4 \beta_{X Y} \beta_{X^{3} Y}-\beta_{X^{4}}\left(1+\beta_{X^{2}}\right)\right)+2 \beta_{X^{3} Y}^{2}+\beta_{X^{4}}\left(\beta_{X^{4}}+2 \beta_{X Y}^{2}\right), \\
& G=2 \beta_{X Y}\left(\beta_{X Y}-2 \beta_{X^{3} Y}\right)+\beta_{X Y X Y}\left(2 \beta_{X^{2}}-1-\beta_{X^{4}}\right)+\beta_{X^{2}}\left(2 \beta_{X^{2}}-1-3 \beta_{X^{4}}\right)+2 \beta_{X^{3} Y}^{2}+\beta_{X^{4}}\left(1+\beta_{X^{4}}\right) .
\end{aligned}
$$

Let $\alpha_{0}>0$ be the smallest positive number such that the rank of $B\left(\alpha_{0}\right)$ is smaller than 6. By (7.8) we get

$$
\alpha_{0}=\min \left(\frac{\beta_{X Y}^{2}-\beta_{X^{2}}+\beta_{X^{2}}^{2}}{2\left(-1+\beta_{X^{2}}\right)}, \frac{F}{2 G}\right) \text {. }
$$

Claim 1: $\alpha_{0}=\frac{F}{2 G}<\alpha_{1}$. 
Since

$$
\begin{aligned}
\operatorname{det}\left(\left.B(\alpha)\right|_{\left\{\mathbb{1}, \mathbb{X}^{2}\right\}}\right) & =\beta_{X^{4}}-\beta_{X^{2}}^{2}+2 \alpha\left(-1+2 \beta_{X^{2}}-\beta_{X^{4}}\right), \\
\operatorname{det}\left(\left.B(\alpha)\right|_{\{\mathbb{1}, \mathbb{X}\}}\right) & =C-\beta_{X Y}^{2}-2 \alpha \cdot C, \\
\operatorname{det}\left(\left.B(\alpha)\right|_{\{\mathbb{1}, \mathbb{X} \mathbb{Y}, \mathbb{Y}\}}\right) & =(E-C)\left(-E-C+2 \beta_{X^{2} Y}+2 \alpha \cdot(E+C)\right),
\end{aligned}
$$

the system

$$
\operatorname{det}\left(\left.B\left(\alpha_{2}\right)\right|_{\left\{\mathbb{1}, \mathbb{X}^{2}\right\}}\right)=0, \quad \operatorname{det}\left(\left.B\left(\alpha_{3}\right)\right|_{\{\mathbb{1}, \mathbb{X} \mathbb{Y}\}}\right)=0, \quad \operatorname{det}\left(\left.B\left(\alpha_{4}\right)\right|_{\{\mathbb{1}, \mathbb{X} \mathbb{Y}, \mathbb{Y X}\}}\right)=0
$$

has a solution

$$
\alpha_{2}=\frac{\beta_{X^{2}}^{2}-\beta_{X^{4}}}{2\left(-1+2 \beta_{X^{2}}-\beta_{X^{4}}\right)}, \quad \alpha_{3}=\frac{-\beta_{X Y}^{2}+\beta_{X^{2}}-\beta_{X^{4}}}{2\left(\beta_{X^{2}}-\beta_{X^{4}}\right)}, \quad \alpha_{4}=\frac{-2 \beta_{X Y}^{2}+\beta_{X Y X Y}+\beta_{X^{2}}-\beta_{X^{4}}}{2\left(\beta_{X Y X Y}+\beta_{X^{2}}-\beta_{X^{4}}\right)} .
$$

If $\alpha_{1} \leq \frac{F}{2 G}$, then since $B\left(\alpha_{1}\right) \succeq 0$, it follows that $\alpha_{1} \leq \min \left(\alpha_{2}, \alpha_{3}, \alpha_{4}\right)$. Using Mathematica, the system

$$
\begin{array}{r}
\alpha_{1} \leq \min \left(\alpha_{2}, \alpha_{3}, \alpha_{4}\right), \quad \operatorname{det}\left(\left.\mathcal{M}_{2}\right|_{\{\mathbb{Y}\}}\right)>0, \quad \operatorname{det}\left(\left.\mathcal{M}_{2}\right|_{\{\mathbb{X} \mathbb{Y}\}}\right)>0, \\
\operatorname{det}\left(\left.\mathcal{M}_{2}\right|_{\{\mathbb{X}, \mathbb{Y}\}}\right)>0, \quad \operatorname{det}\left(\left.\mathcal{M}_{2}\right|_{\left\{1, X^{2}\right\}}\right)>0, \quad \operatorname{det}\left(\left.\mathcal{M}_{2}\right|_{\{1, \mathbb{X} \mathbb{Y}, \mathbb{X}\}}\right)>0,
\end{array}
$$

does not have solutions (see https://github.com/Abhishek-B/TTMP for the Mathematica file). Hence $\alpha_{0}=\frac{F}{2 G}<\alpha_{1}$.

Using Mathematica to calculate the kernel of $B\left(\frac{F}{2 G}\right)$ we conclude that $B\left(\frac{F}{2 G}\right)$ satisfies the relations

$$
\mathbb{X} \mathbb{Y}+\mathbb{Y}=a \mathbb{1}+d \mathbb{X}^{2}, \quad \mathbb{Y}^{2}+\mathbb{X}^{2}=\mathbb{1}
$$

for some $a, d \in \mathbb{R}$. We also have

$$
\beta_{X}^{(B)}=\beta_{Y}^{(B)}=\beta_{X^{3}}^{(B)}=\beta_{X^{2} Y}^{(B)}=\beta_{X Y^{2}}^{(B)}=\beta_{Y^{3}}^{(B)}=0,
$$

where $\beta_{w(X, Y)}^{(B)}$ are the moments of $B\left(\frac{F}{2 G}\right)$. This is a special case in the proof of Proposition 4.1, i.e., Case 2.2. Following the proof we see that after using only transformations of type

$$
(x, y) \mapsto\left(\alpha_{1} x+\beta_{1} y, \alpha_{2} x+\beta_{2} y\right)
$$

for some $\alpha_{1}, \alpha_{2}, \beta_{1}, \beta_{2} \in \mathbb{R}$, we come into the basic case 1 or 2 of rank 5 with $\widetilde{\beta}_{X}=\widetilde{\beta}_{Y}=\widetilde{\beta}_{X^{3}}=0$. But every such sequence admits a measure of type $(2,1)$ by Theorems 6.5 and 6.8 . Hence $\beta$ admits a measure of type $(4,1)$.

It remains to prove (2). Suppose that $\beta$ admits a nc measure. By Proposition 7.3 and Theorem 7.5 (1),

$$
\mathcal{M}_{2}=\sum_{i} \lambda_{i} \mathcal{M}^{\left(x_{i}, y_{i}\right)}(2)+\xi \mathcal{M}^{(X, Y)}(2),
$$

where $\left(x_{i}, y_{i}\right) \in \mathbb{R}^{2},(X, Y) \in\left(\mathbb{S R}^{2 \times 2}\right)^{2}, \lambda_{i}>0, \xi>0$ and $\sum_{i} \lambda_{i}+\xi=1$. Therefore

$$
\mathcal{M}_{2}-\xi \mathcal{M}_{2}^{(X, Y)}
$$

is a $\mathrm{cm}$ moment matrix satisfying the relations

$$
\mathbb{Y}^{2}=\mathbb{1}-\mathbb{X}^{2} \quad \text { and } \quad \mathbb{X} \mathbb{Y}=\mathbb{Y} \mathbb{X}
$$

By Theorem 2.7, $M$ admits a measure if and only if $M$ is psd and satisfies rank $M \leq \operatorname{card} \mathcal{V}_{M}$. To conclude the proof it only remains to prove that $X, Y$ are of the form (7.7). $\mathcal{M}_{2}^{(X, Y)}$ is a nc moment matrix rank 4. Therefore the columns $\{\mathbb{1}, \mathbb{X}, \mathbb{Y}, \mathbb{X} \mathbb{Y}\}$ are linearly independent and hence

$$
\mathbb{X}^{2}=a_{1} \mathbb{1}+b_{1} \mathbb{X}+c_{1} \mathbb{Y}+d_{1} \mathbb{X} \mathbb{Y}, \quad \text { and } \quad \mathbb{Y}^{2}=a_{3} \mathbb{1}+b_{3} \mathbb{X}+c_{3} \mathbb{Y}+d_{3} \mathbb{X} \mathbb{Y},
$$

where $a_{j}, b_{j}, c_{j}, d_{j} \in \mathbb{R}$ for $j=1,3$. By Theorem $3.1(1), d_{1}=d_{3}=0$. By Theorem $3.1(3), c_{1}=b_{3}=0$. Since $\mathbb{X}^{2}+\mathbb{Y}^{2}=\mathbb{1}$ it follows that $b_{1}=c_{3}=0$ and $a_{3}=1-a_{1}$. By Theorem 3.1 (4), $X$ and $Y$ are of the form (7.7).

The following theorem translates the BQTMP for $\beta$ with $\mathcal{M}_{2}$ of rank 6 satisfying $\mathbb{Y}^{2}=\mathbb{1}-\mathbb{X}^{2}$ into the feasibility problem of some LMIs and a rank-to-cardinality condition from Theorem 2.7. 
Corollary 7.6. Suppose $\beta \equiv \beta^{(4)}$ is a normalized nc sequence with a moment matrix $\mathcal{M}_{2}$ of rank 6 satisfying the relation $\mathbb{Y}^{2}=\mathbb{1}-\mathbb{X}^{2}$. Let $L(a, b, c, d, e)$ be the following linear matrix polynomial

$$
\left(\begin{array}{ccccccc}
a & \beta_{X} & \beta_{Y} & b & c & c & a-b \\
\beta_{X} & b & c & \beta_{X^{3}} & \beta_{X^{2} Y} & \beta_{X^{2} Y} & \beta_{X}-\beta_{X^{3}} \\
\beta_{Y} & c & a-b & \beta_{X^{2} Y} & \beta_{X}-\beta_{X^{3}} & \beta_{X}-\beta_{X^{3}} & \beta_{Y}-\beta_{X^{2} Y} \\
b & \beta_{X^{3}} & \beta_{X^{2} Y} & d & e & e & b-d \\
c & \beta_{X^{2} Y} & \beta_{X}-\beta_{X^{3}} & e & b-d & b-d & c-e \\
c & \beta_{X^{2} Y} & \beta_{X}-\beta_{X^{3}} & e & b-d & b-d & c-e \\
a-b & \beta_{X}-\beta_{X^{3}} & \beta_{Y}-\beta_{X^{2} Y} & b-d & c-e & c-e & a-2 b+d
\end{array}\right)
$$

where $a, b, c, d, e \in \mathbb{R}$. Then $\beta$ admits a nc measure if and only if there exist $a, b, c, d, e \in \mathbb{R}$ such that

(1) $L(a, b, c, d, e) \succeq 0$,

(2) $\mathcal{M}_{2}-L(a, b, c, d, e) \succeq 0$,

(3) $\left(\mathcal{M}_{2}-L(a, b, c, d, e)\right)_{\{\mathbb{1}, \mathbb{X}, \mathbb{Y}, \mathbb{X} \mathbb{Y}\}} \succ 0$,

(4) $\operatorname{rank}(L(a, b, c, d, e)) \leq$ card $\mathcal{V}_{L}$, where $\mathcal{V}_{L}$ is the variety associated to the moment matrix $L(a, b, c, d, e)$ (see Theorem 2.7).

Proof. By Theorem 7.5, $\beta$ admits a nc measure if and only if

$$
\mathcal{M}_{2}=\sum_{i=1}^{k} \lambda_{i} \mathcal{M}_{2}^{\left(x_{i}, y_{i}\right)}+\xi \mathcal{M}_{2}^{(X, Y)},
$$

where $\left(x_{i}, y_{i}\right) \in \mathbb{R}^{2},(X, Y) \in\left(\mathbb{S R}^{2 \times 2}\right)^{2}, \lambda_{i}>0, \xi>0$ and $\sum_{i} \lambda_{i}+\xi=1$. By Corollary 3.2,

$$
\beta_{X}^{(X, Y)}=\beta_{Y}^{(X, Y)}=\beta_{X^{3}}^{(X, Y)}=\beta_{X^{2} Y}^{(X, Y)}=\beta_{X Y^{2}}^{(X, Y)}=\beta_{Y^{3}}^{(X, Y)}=0,
$$

where $\beta_{w(X, Y)}^{(X, Y)}$ are the moments of $\mathcal{M}_{2}^{(X, Y)}$. Using (7.14) and (7.15), we conclude that $\sum_{i} \lambda_{i} \mathcal{M}_{2}^{\left(x_{i}, y_{i}\right)}$ and $\xi \mathcal{M}_{2}^{(X, Y)}$ are of the forms

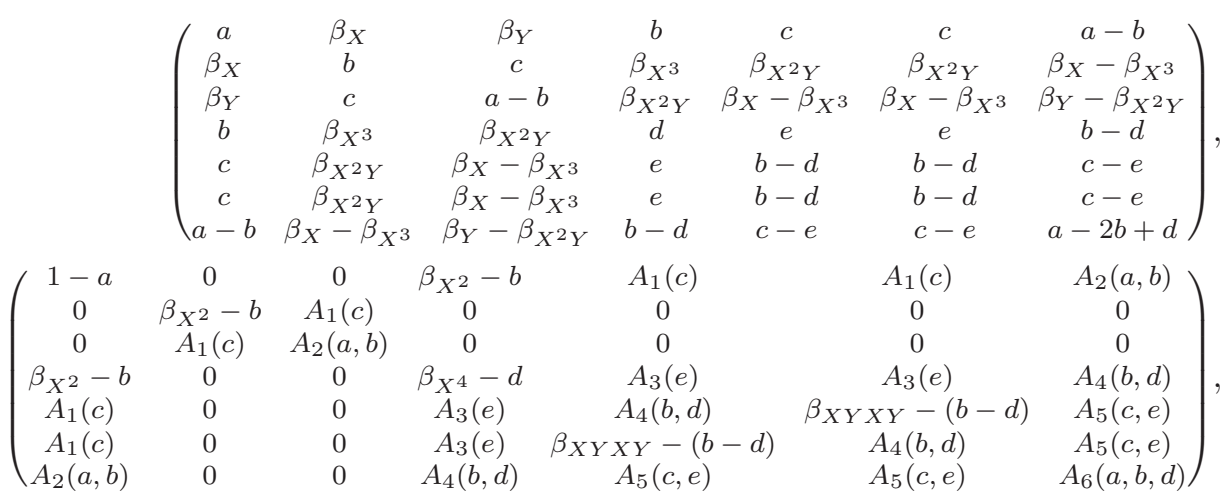

where

$$
\begin{aligned}
A_{1}(c) & =\beta_{X Y}-c, \quad A_{2}(a, b)=1-\beta_{X^{2}}-(a-b), \\
A_{3}(e) & =\beta_{X^{3} Y}-e, \quad A_{4}(b, d)=\beta_{X^{2}}-\beta_{X^{4}}-(b-d), \\
A_{5}(c, e) & =\beta_{X Y}-\beta_{X^{3} Y}-(c-e), \quad A_{6}(a, b, d)=1-2 \beta_{X^{2}}+\beta_{X^{4}}-(a-2 b+d),
\end{aligned}
$$

for some $a, b, c, d, e \in \mathbb{R}$. Notice that the matrix (7.16) equals to $L(a, b, c, d, e)$ and the matrix (7.17) to $\mathcal{M}_{2}-$ $L(a, b, c, d, e)$. Since $L(a, b, c, d, e)$ is a $\mathrm{cm}$ moment matrix, it admits a nc measure by Theorem 2.7 if and only if (1) and (4) of Theorem 7.6 are true. Since $\mathcal{M}_{2}-L(a, b, c, d, e)$ is a nc moment matrix satisfying $\mathbb{Y}^{2}=\mathbb{1}-\mathbb{X}^{2}$ and $\widetilde{\beta}_{X}=\widetilde{\beta}_{Y}=\widetilde{\beta}_{X^{3}}=\widetilde{\beta}_{X^{2} Y}=\widetilde{\beta}_{X Y^{2}}=\widetilde{\beta}_{Y^{3}}=0$, it admits a nc measure by the results of rank 4 and 5 cases and Theorem 7.5 (1) if and only if (2) and (3) of Theorem 7.6 are true.

7.2. Relation $\mathbb{X} \mathbb{Y}+\mathbb{Y} \mathbb{X}=\mathbf{0}$. In this subsection we study a nc sequence $\beta \equiv \beta^{(4)}$ with a moment matrix $\mathcal{M}_{2}$ of rank 6 satisying the relation $\mathbb{X} \mathbb{Y}+\mathbb{Y} \mathbb{X}=\mathbf{0}$. In Theorem 7.8 we characterize when $\beta$ admits a nc measure. In Corollary 7.9 we show that the existence of a nc measure is equivalent to the feasibility problem of three LMIs and a rank-to-variety condition from Theorem 2.7.

The form of $\mathcal{M}_{2}$ is given by the following proposition. 
Proposition 7.7. Let $\beta \equiv \beta^{(4)}$ be a nc sequence with a moment matrix $\mathcal{M}_{2}$ of rank 6 satisfying the relation

$$
\mathbb{X} \mathbb{Y}+\mathbb{Y} \mathbb{X}=\mathbf{0}
$$

Then $\mathcal{M}_{2}$ is of the form

$$
\left(\begin{array}{ccccccc}
\beta_{1} & \beta_{X} & \beta_{Y} & \beta_{X^{2}} & 0 & 0 & \beta_{Y^{2}} \\
\beta_{X} & \beta_{X^{2}} & 0 & \beta_{X^{3}} & 0 & 0 & 0 \\
\beta_{Y} & 0 & \beta_{Y^{2}} & 0 & 0 & 0 & \beta_{Y^{3}} \\
\beta_{X^{2}} & \beta_{X^{3}} & 0 & \beta_{X^{4}} & 0 & 0 & \beta_{X^{2} Y^{2}} \\
0 & 0 & 0 & 0 & \beta_{X^{2} Y^{2}} & -\beta_{X^{2} Y^{2}} & 0 \\
0 & 0 & 0 & 0 & -\beta_{X^{2} Y^{2}} & \beta_{X^{2} Y^{2}} & 0 \\
\beta_{Y^{2}} & 0 & \beta_{Y^{3}} & \beta_{X^{2} Y^{2}} & 0 & 0 & \beta_{Y^{4}}
\end{array}\right) .
$$

Proof. The relation (7.20) gives us the following system in $\mathcal{M}_{2}$

$$
\begin{array}{rrr}
2 \beta_{X Y}=0, & 2 \beta_{X^{3} Y}=0, \\
2 \beta_{X^{2} Y}=0, & \beta_{X^{2} Y^{2}}+\beta_{X Y X Y}=\beta_{X Y}, \\
2 \beta_{X Y^{2}}=0, & 2 \beta_{X Y^{3}}=0 .
\end{array}
$$

Thus the solution of the system (7.22) is given by the statement of the proposition.

The following theorem characterizes normalized sequences $\beta$ with a moment matrix $\mathcal{M}_{2}$ of rank 6 satisfying $\mathbb{X} \mathbb{Y}+$ $\mathbb{Y} \mathbb{X}=\mathbf{0}$, which admit a nc measure.

Theorem 7.8. Suppose $\beta \equiv \beta^{(4)}$ is a normalized nc sequence with a moment matrix $\mathcal{M}_{2}$ of rank 6 satisfying the relation $\mathbb{X} \mathbb{Y}+\mathbb{Y} \mathbb{X}=\mathbf{0}$. Then $\beta$ admits a nc measure if and only if $\mathcal{M}_{2}$ is positive semidefinite and one of the following is true:

(1) $\beta_{X}=\beta_{Y}=\beta_{X^{3}}=\beta_{Y^{3}}=0$. There exists a nc measure of type $(2,1)$ or $(3,1)$.

(2) There exist

$$
a_{1}>0, \quad a_{3}>0
$$

such that

$$
M:=\mathcal{M}_{2}-\xi \mathcal{M}_{2}^{(X, Y)}
$$

is a positive semidefinite cm moment matrix satisfying $\operatorname{rank} M \leq \operatorname{card} \mathcal{V}_{M}$, where $\mathcal{V}_{M}$ is the variety associated to $M$ (as in Theorem 2.7),

$$
X=\left(\begin{array}{cc}
\sqrt{a_{1}} & 0 \\
0 & -\sqrt{a_{1}}
\end{array}\right), \quad Y=\left(\begin{array}{cc}
0 & \sqrt{a_{3}} \\
\sqrt{a_{3}} & 0
\end{array}\right)
$$

and $\xi>0$ is the smallest positive number such that rank of $\mathcal{M}_{2}-\xi \mathcal{M}_{2}^{(X, Y)}$ is smaller than the rank of $\mathcal{M}_{2}$.

Proof. First we will prove (1). $\mathcal{M}_{2}$ is of the form

We define the matrix function

$$
\mathcal{M}_{2}=\left(\begin{array}{ccccccc}
1 & 0 & 0 & \beta_{X^{2}} & 0 & 0 & \beta_{Y^{2}} \\
0 & \beta_{X^{2}} & 0 & 0 & 0 & 0 & 0 \\
0 & 0 & \beta_{Y^{2}} & 0 & 0 & 0 & 0 \\
\beta_{X^{2}} & 0 & 0 & \beta_{X^{4}} & 0 & 0 & \beta_{X^{2} Y^{2}} \\
0 & 0 & 0 & 0 & \beta_{X^{2} Y^{2}} & -\beta_{X^{2} Y^{2}} & 0 \\
0 & 0 & 0 & 0 & -\beta_{X^{2} Y^{2}} & \beta_{X^{2} Y^{2}} & 0 \\
\beta_{Y^{2}} & 0 & 0 & \beta_{X^{2} Y^{2}} & 0 & 0 & \beta_{Y^{4}}
\end{array}\right) .
$$

We have that

$$
B(\alpha):=\mathcal{M}_{2}-\alpha \mathcal{M}_{2}^{(0,0)}
$$

where

$$
\operatorname{det}\left(\left.B(\alpha)\right|_{\left\{\mathbb{1}, \mathbb{X}, \mathbb{Y}, \mathbb{X}^{2}, \mathbb{X} \mathbb{Y}, \mathbb{Y}^{2}\right\}}\right)=-\beta_{Y^{2}} \beta_{X^{2}} \beta_{X^{2} Y^{2}}(F-\alpha G),
$$

$$
F=\beta_{Y^{4}} \beta_{X^{2}}^{2}-2 \beta_{Y^{2}} \beta_{X^{2}} \beta_{X^{2} Y^{2}}+\beta_{X^{2} Y^{2}}+\beta_{Y^{2}}^{2} \beta_{X^{4}}-\beta_{X^{4}} \beta_{Y^{4}}, \quad G=\beta_{X^{2} Y^{2}}^{2}-\beta_{X^{4}} \beta_{Y^{4}} .
$$

Let $\alpha_{0}>0$ be the smallest positive number such that the rank of $B\left(\alpha_{0}\right)$ is smaller than 6. By (7.24) we get $\alpha_{0}=\frac{F}{G}$. The kernel of $B\left(\alpha_{0}\right)$ satisfies the relations

$$
\mathbb{Y}^{2}=\frac{\beta_{X^{4}} \beta_{Y^{4}}-\beta_{X^{2} Y^{2}}^{2}}{\beta_{Y^{2}} \beta_{X^{4}}-\beta_{X^{2}} \beta_{X^{2} Y^{2}}} \mathbb{1}+\frac{\beta_{Y^{2}} \beta_{X^{2} Y^{2}}-\beta_{Y^{4}} \beta_{X^{2}}}{\beta_{Y^{2}} \beta_{X^{4}}-\beta_{X^{2}} \beta_{X^{2} Y^{2}}} \mathbb{X}^{2}, \quad \mathbb{X} \mathbb{Y}+\mathbb{Y} \mathbb{X}
$$


It also satisfies

$$
\beta_{X}^{(B)}=\beta_{Y}^{(B)}=\beta_{X Y}^{(B)}=\beta_{X^{3}}^{(B)}=\beta_{X^{2} Y}^{(B)}=\beta_{X Y^{2}}^{(B)}=\beta_{Y^{3}}^{(B)}=0
$$

where $\beta_{w(X, Y)}^{(B)}$ are the moments of $B\left(\alpha_{0}\right)$. This is a special case in the proof of Proposition 4.1 (1), i.e., Case 2.3. Following the proof we see that after using only transformations of type

$$
(x, y) \mapsto\left(\alpha_{1} x+\beta_{1} y, \alpha_{2} x+\beta_{2} y\right)
$$

for some $\alpha_{1}, \alpha_{2}, \beta_{1}, \beta_{2} \in \mathbb{R}$, we come into one of the basic pairs 1 or 4 of rank 5 with $\widetilde{\beta}_{X}=\widetilde{\beta}_{Y}=\widetilde{\beta}_{X^{3}}=0$. But every such moment matrix admits a measure of type $(1,1)$ or $(2,1)$ by Theorems 6.5 and 6.14 . Hence $\mathcal{M}_{2}$ admits a nc measure of type $(2,1)$ or $(3,1)$.

It remains to prove (2). Suppose that $\beta$ admits a nc measure. By Propositions 7.3 and Theorem 7.8 (1),

$$
\mathcal{M}_{2}=\sum_{i} \lambda_{i} \mathcal{M}_{2}^{\left(x_{i}, y_{i}\right)}+\xi \mathcal{M}_{2}^{(X, Y)}
$$

where $\left(x_{i}, y_{i}\right) \in \mathbb{R}^{2},(X, Y) \in\left(\mathbb{S R}^{2 \times 2}\right)^{2}, \lambda_{i}>0, \xi>0$ and $\sum_{i} \lambda_{i}+\xi=1$. Therefore

$$
M:=\mathcal{M}_{2}-\xi \mathcal{M}_{2}^{(X, Y)}
$$

is a $\mathrm{cm}$ moment matrix satisfying the relations

$$
\mathbb{X} \mathbb{Y}+\mathbb{Y}=\mathbf{0} \text { and } \mathbb{X} \mathbb{Y}=\mathbb{Y} \mathbb{X} \text {. }
$$

By Theorem 2.7, $M$ admits a nc measure if and only if $M$ is psd and satisfies rank $M \leq \operatorname{card} \mathcal{V}_{M}$. To conclude the proof it only remains to prove that $X, Y$ are of the form (7.23). $\mathcal{M}_{2}^{(X, Y)}$ is a nc moment matrix of rank 4. Therefore the columns $\{\mathbb{1}, \mathbb{X}, \mathbb{Y}, \mathbb{X} \mathbb{Y}\}$ are linearly independent and hence

$$
\mathbb{X}^{2}=a_{1} \mathbb{1}+b_{1} \mathbb{X}+c_{1} \mathbb{Y}+d_{1} \mathbb{X} \mathbb{Y} \quad \text { and } \quad \mathbb{Y}^{2}=a_{3} \mathbb{1}+b_{3} \mathbb{X}+c_{3} \mathbb{Y}+d_{3} \mathbb{X} \mathbb{Y} .
$$

where $a_{j}, b_{j}, c_{j}, d_{j} \in \mathbb{R}$ for $j=1,2,3$. By Theorem $3.1(1), d_{1}=d_{3}=0$. By Theorem $3.1(3), c_{1}=b_{3}=0$. Since $\mathbb{X} \mathbb{Y}+\mathbb{Y} \mathbb{X}=\mathbf{0}$ it follows that $b_{1}=c_{3}=0$. By Theorem 3.1 (4), $X$ and $Y$ are of the form (7.23).

The following corollary translates the BQTMP for $\beta$ with $\mathcal{M}_{2}$ of rank 6 satisying $\mathbb{X} \mathbb{Y}+\mathbb{Y} \mathbb{X}=\mathbf{0}$ into the feasibility problem of some LMIs and a rank-to-variety condition from Theorem 2.7.

Corollary 7.9. Suppose $\beta \equiv \beta^{(4)}$ is a normalized nc sequence with a moment matrix $\mathcal{M}_{2}$ of rank 6 satisfying the relation $\mathbb{X} \mathbb{Y}+\mathbb{Y} \mathbb{X}=\mathbf{0}$. Let us define a linear matrix polynomial

$$
L(a, b, c, d, e)=\left(\begin{array}{ccccccc}
a & \beta_{X} & \beta_{Y} & b & 0 & 0 & c \\
\beta_{X} & b & 0 & \beta_{X^{3}} & 0 & 0 & 0 \\
\beta_{Y} & 0 & c & 0 & 0 & 0 & \beta_{Y^{3}} \\
b & \beta_{X^{3}} & 0 & d & 0 & 0 & 0 \\
0 & 0 & 0 & 0 & 0 & 0 & 0 \\
0 & 0 & 0 & 0 & 0 & 0 & 0 \\
c & 0 & \beta_{Y^{3}} & 0 & 0 & 0 & e
\end{array}\right),
$$

where $a, b, c, d, e \in \mathbb{R}$. Then $\beta$ admits a nc measure if and only there exist

$$
a \in(0,1), \quad b \in\left(0, \beta_{X^{2}}\right), \quad c \in\left(0, \beta_{Y^{2}}\right), \quad d \in\left(0, \beta_{X^{4}}\right), \quad e \in\left(0, \beta_{Y^{4}}\right),
$$

such that

(1) $L(a, b, c, d, e) \succeq 0$,

(2) $\mathcal{M}_{2}-L(a, b, c, d, e) \succeq 0$,

(3) $\operatorname{rank}(L(a, b, c, d, e)) \leq \operatorname{card} \mathcal{V}_{L}$, where $\mathcal{V}_{L}$ is the variety associated to the moment matrix $L(a, b, c, d, e)$ (see Theorem 2.7).

Proof. By Theorem 7.8, $\beta$ admits a nc measure if and only if

$$
\mathcal{M}_{2}=\sum_{i=1}^{k} \lambda_{i} \mathcal{M}_{2}^{\left(x_{i}, y_{i}\right)}+\xi \mathcal{M}_{2}^{(X, Y)},
$$

where $\left(x_{i}, y_{i}\right) \in \mathbb{R}^{2},(X, Y) \in\left(\mathbb{S R}^{2 \times 2}\right)^{2}, \lambda_{i}>0, \xi>0$ and $\sum_{i} \lambda_{i}+\xi=1$. By Corollary 3.2,

$$
\beta_{X}^{(X, Y)}=\beta_{Y}^{(X, Y)}=\beta_{X^{3}}^{(X, Y)}=\beta_{X^{2} Y}^{(X, Y)}=\beta_{X Y^{2}}^{(X, Y)}=\beta_{Y^{3}}^{(X, Y)}=0
$$


where $\beta_{w(X, Y)}^{(X, Y)}$ are the moments of $\mathcal{M}_{2}^{(X, Y)}$. Since $\mathbb{X} \mathbb{Y}+\mathbb{Y} \mathbb{X}=\mathbf{0}$, we also have $\beta_{X Y}^{(X, Y)}=0$. Using (7.26) and (7.27), we conclude that $\sum_{i} \lambda_{i} \mathcal{M}_{2}^{\left(x_{i}, y_{i}\right)}$ and $\xi \mathcal{M}_{2}^{(X, Y)}$ are of the forms

$$
\begin{aligned}
& \left(\begin{array}{ccccccc}
a & \beta_{X} & \beta_{Y} & b & 0 & 0 & c \\
\beta_{X} & b & 0 & \beta_{X^{3}} & 0 & 0 & 0 \\
\beta_{Y} & 0 & c & 0 & 0 & 0 & \beta_{Y^{3}} \\
b & \beta_{X^{3}} & 0 & d & 0 & 0 & 0 \\
0 & 0 & 0 & 0 & 0 & 0 & 0 \\
0 & 0 & 0 & 0 & 0 & 0 & 0 \\
c & 0 & \beta_{Y^{3}} & 0 & 0 & 0 & e
\end{array}\right) \\
& \left(\begin{array}{ccccccc}
1-a & 0 & 0 & \beta_{X^{2}}-b & 0 & 0 & \beta_{Y^{2}}-c \\
0 & \beta_{X^{2}}-b & 0 & 0 & 0 & 0 & 0 \\
0 & 0 & \beta_{Y^{2}}-c & 0 & 0 & 0 & 0 \\
\beta_{X^{2}}-b & 0 & 0 & \beta_{X^{4}}-d & 0 & 0 & \beta_{X^{2} Y^{2}} \\
0 & 0 & 0 & 0 & \beta_{X^{2} Y^{2}} & -\beta_{X^{2} Y^{2}} & 0 \\
0 & 0 & 0 & 0 & -\beta_{X^{2} Y^{2}} & \beta_{X^{2} Y^{2}} & 0 \\
\beta_{Y^{2}}-c & 0 & 0 & \beta_{X^{2} Y^{2}} & 0 & 0 & \beta_{Y^{4}}-e
\end{array}\right),
\end{aligned}
$$

for some $a, b, c, d, e \in \mathbb{R}$. Notice that the matrix (7.28) is $L(a, b, c, d, e)$ and the matrix (7.29) is $\mathcal{M}_{2}-L(a, b, c, d, e)$. Since $L(a, b, c, d, e)$ is a cm moment matrix, it admits a nc measure by Theorem 2.7 if and only if (1) and (3) of Corollary 7.9 are true. Since $\mathcal{M}_{2}-L(a, b, c, d, e)$ is a nc moment matrix satisfying

$$
\mathbb{X} \mathbb{Y}+\mathbb{Y} \mathbb{X}=0 \text { and } \widetilde{\beta}_{X}=\widetilde{\beta}_{Y}=\widetilde{\beta}_{X^{3}}=\widetilde{\beta}_{Y^{3}}=0,
$$

it admits a nc measure by the results of rank 4 and 5 cases and Theorem 7.8 (1) if and only if (2) of Corollary 7.9 is true.

\section{Flat eXtensions $\mathcal{M}_{3}$ FOR THE BQTMP with $\mathcal{M}_{2}$ OF RANK 6}

In this section we characterize when a nc sequence $\beta \equiv \beta^{(4)}$ with a moment matrix $\mathcal{M}_{2}$ of rank 6 satisfying one of the basic relations of Proposition 4.1 (2), admits a flat extension to a moment matrix $\mathcal{M}_{3}$. By Theorem 2.5, this is a sufficient condition for the existence of a nc measure. We demonstrate with examples that this is not a necessary condition for none of the basic relations of Proposition 4.1 (2). This shows a difference with the $\mathrm{cm}$ case since by Theorem 2.7 (3), if $\beta$ is a $\mathrm{cm}$ sequence with a psd moment matrix $\mathcal{M}_{2}$ satisfying exactly one of the relations $x^{2}+y^{2}=1, x^{2}-y^{2}=1$ (since it is equivalent to $x y=1$ ) or $y^{2}=1$, then $\mathcal{M}_{2}$ admits a flat extension to a moment matrix $\mathcal{M}_{3}$. We can also conclude that $\mathcal{M}_{2}$ being psd does not imply that there exist $a, b, c, d, e \in \mathbb{R}$ in Corollary 7.6 such that (1)-(4) hold.

8.1. Preliminaries. In this subsection we introduce some preliminaries needed in solving the flat extension question. First we establish the form of an extension $\mathcal{M}$ of a moment matrix $\mathcal{M}_{2}$ to be a moment matrix of degree 3 .

Proposition 8.1. Suppose $\mathcal{M}_{3}=\left(\begin{array}{cc}\mathcal{M}_{2} & B_{3} \\ B_{3}^{t} & C_{3}\end{array}\right)$ is a moment matrix of degree 3 , where $B_{3} \in \mathbb{R}^{7 \times 8}$ and $C_{3} \in \mathbb{R}^{8 \times 8}$ and let the rows and columns be order lexicographically. Then $B_{3}$ and $C_{3}$ are of the forms

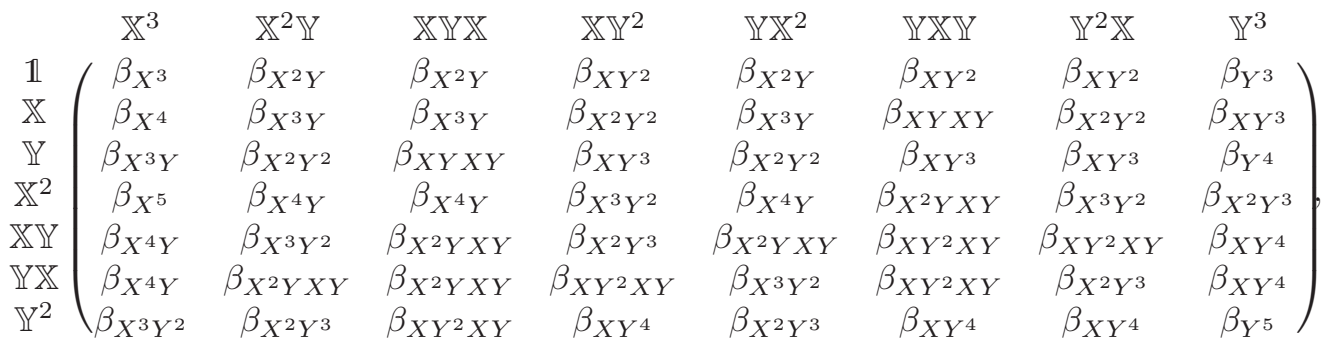


THE SINGULAR BIVARIATE QUARTIC TRACIAL MOMENT PROBLEM

\begin{tabular}{|c|c|c|c|c|c|c|c|c|}
\hline & $\mathbb{X}^{3}$ & $\mathbb{X}^{2} \mathbb{Y}$ & $\mathbb{X} \mathbb{Y}$ & $\mathbb{X} \mathbb{Y}^{2}$ & $\mathbb{Y X}^{2}$ & $\mathbb{Y} \mathbb{X} \mathbb{Y}$ & $\mathbb{Y}^{2} \mathbb{X}$ & $\mathbb{Y}^{3}$ \\
\hline $\mathbb{Z} 3$ & $\beta_{X^{6}}$ & $\beta_{X^{5} Y}$ & $\beta_{X^{5} Y}$ & $\beta_{X^{4} Y^{2}}$ & $\beta_{X^{5} Y}$ & $\beta_{X^{3} Y X Y}$ & $\beta_{X^{4} Y^{2}}$ & $\beta_{X^{3} Y^{3}}$ \\
\hline I & $\beta_{X^{5} Y}$ & $\beta_{X^{4} Y^{2}}$ & $\beta_{X^{3} Y X Y}$ & $\beta_{X^{3} Y^{3}}$ & $\beta_{X^{2} Y X^{2} Y}$ & $\beta_{X^{2} Y^{2} X Y}$ & $\beta_{X^{2} Y^{2} X Y}$ & $\beta_{X^{2} Y^{4}}$ \\
\hline $\mathbb{Y} \mathbb{X}$ & $\beta_{X^{4} Y X}$ & $\beta_{X^{3} Y X Y}$ & $\beta_{X^{2} Y X^{2} Y}$ & $\beta_{X^{2} Y^{2} X Y}$ & $\beta_{X^{3} Y X Y}$ & $\beta_{X Y X Y X Y}$ & $\beta_{X^{2} Y^{2} X Y}$ & $\beta_{X Y^{3} X Y}$ \\
\hline $\mathbb{X} \mathbb{Y}^{2}$ & $\beta_{X^{4} Y^{2}}$ & $\beta_{X^{3} Y^{3}}$ & $\beta_{X^{2} Y^{2} X Y}$ & $\beta_{X^{2} Y^{4}}$ & $\beta_{X^{2} Y^{2} X Y}$ & $\beta_{X Y^{3} X Y}$ & $\beta_{X Y^{2} X Y^{2}}$ & $\beta_{X Y^{5}}$ \\
\hline $\mathbb{Y}^{2}$ & $\beta_{X^{5} Y}$ & $\beta_{X^{2} Y X^{2} Y}$ & $\beta_{X^{3} Y X Y}$ & $\beta_{X^{2} Y^{2} X Y}$ & $\beta_{X^{4} Y^{2}}$ & $\beta_{X^{2} Y^{2} X Y}$ & $\beta_{X^{3} Y^{3}}$ & $\beta_{X^{2} Y^{4}}$ \\
\hline $\begin{array}{l}\mathbb{Y} \mathbb{X} \mathbb{Y} \\
\mathbb{Y} 2 \mathbb{Y}\end{array}$ & $\beta_{X^{3} Y X Y}$ & $\beta_{X^{2} Y^{2} X Y}$ & $\beta_{X Y X Y X Y}$ & $\beta_{X Y^{3} X Y}$ & $\beta_{X^{2} Y^{2} X Y}$ & $\beta_{X Y^{2} X Y^{2}}$ & $\beta_{X Y^{3} X Y}$ & $\beta_{X Y^{5}}$ \\
\hline $\mathbb{Y}^{2} \mathbb{X}$ & $\beta_{X^{4} Y^{2}}$ & $\beta_{X^{2} Y^{2} X Y}$ & $\beta_{X^{2} Y^{2} X Y}$ & $\beta_{X Y^{2} X Y^{2}}$ & $\beta_{X^{3} Y^{3}}$ & $\beta_{X Y^{3} X Y}$ & $\beta_{X^{2} Y^{4}}$ & $\beta_{X Y^{5}}$ \\
\hline $\mathbb{Y}^{3}$ & $\beta_{X^{3} Y^{3}}$ & $\beta_{X^{2} Y^{4}}$ & $\beta_{X Y^{3} X Y}$ & $\beta_{X Y^{5}}$ & $\beta_{X^{2} Y^{4}}$ & $\beta_{X Y^{5}}$ & $\beta_{X Y^{5}}$ & . \\
\hline
\end{tabular}

respectively.

Proof. This follows by definition of moment matrices.

If $B_{3}$ and $C_{3}$ are of the form given in Proposition 8.1, then we say they have a moment structure.

The moment structure of $C_{3}$ implies the system given by the following proposition is satisfied.

Proposition 8.2. If $\mathcal{M}_{3}$ is a moment matrix, then $C_{3}:=\left(C_{i j}\right)_{i j}$ satisfies the following system

$$
\begin{aligned}
C_{47}=C_{66}, & C_{38}=C_{46}=C_{67}, \\
C_{25}=C_{33}, & C_{48}=C_{68}=C_{78}, \\
C_{12}=C_{13}=C_{15}, & C_{14}=C_{17}=C_{22}=C_{55}, \\
C_{18}=C_{24}=C_{57}, & C_{28}=C_{58}=C_{44}=C_{77}, \\
C_{16}=C_{23}=C_{35}, & C_{26}=C_{27}=C_{34}=C_{37}=C_{45}=C_{56} .
\end{aligned}
$$

Recall from Subsection 2.1 that for a polynomial $p \in \mathbb{R}\langle X, Y\rangle_{\leq 2 k}, \hat{p}=\left(a_{w}\right)_{w}$ denotes the coefficient vector with respect to the lexicographically-ordered basis

$$
\left\{1, X, Y, X^{2}, X Y, Y X, Y^{2}, \ldots, X^{2 k}, \ldots, Y^{2 k}\right\}
$$

of $\mathbb{R}\langle X, Y\rangle_{\leq 2 k}$ We will use the following proposition to show that some of the equations in (8.1) are automatically satisfied for any flat extension $\mathcal{M}$ of a moment matrix $\mathcal{M}_{n}$, i.e., $\mathcal{M}$ does not need to have the moment structure.

Proposition 8.3. Suppose $\mathcal{M}=\left(\begin{array}{cc}\mathcal{M}_{n} & B_{n+1} \\ B_{n+1}^{t} & C_{n+1}\end{array}\right)$ is a flat extension of a moment matrix $\mathcal{M}_{n}$ with rows and columns indexed by monomials of degree at most $n+1$. Let $\langle\cdot, \cdot\rangle_{\mathcal{M}}$ be a bilinear form on $\mathbb{R}\langle X, Y\rangle_{\leq n+1}$ defined by $\left\langle w_{1}, w_{2}\right\rangle_{\mathcal{M}}:=$ $\left\langle\mathcal{M} \widehat{w_{1}}, \widehat{w_{2}}\right\rangle$. For polynomials $p, q \in \mathbb{R}\langle X, Y\rangle_{\leq n+1}$ we have

$$
\langle p, q\rangle_{\mathcal{M}}=\langle q, p\rangle_{\mathcal{M}}
$$

and

$$
\langle p, q\rangle_{\mathcal{M}}=\left\langle q^{*}, p^{*}\right\rangle_{\mathcal{M}}
$$

Proof. If $p, q$ are polynomials of degree at most $n$, then (8.2), (8.3) are true due to the moment structure of $\mathcal{M}_{n}$. Suppose $p$ and $q$ be polynomials of degree at most $n+1$. The equality $\langle p, q\rangle_{\mathcal{M}}=\langle q, p\rangle_{\mathcal{M}}$ is true since $\mathcal{M}$ is symmetric. From $\operatorname{rank}(\mathcal{M})=\operatorname{rank}\left(\mathcal{M}_{n}\right)$, it follows that in $\mathcal{M}$ we have

$$
p(\mathbb{X}, \mathbb{Y})=\sum_{|u| \leq n} a_{u} u(\mathbb{X}, \mathbb{Y}) \text { and } q(\mathbb{X}, \mathbb{Y})=\sum_{|t| \leq n} b_{t} t(\mathbb{X}, \mathbb{Y}),
$$

for some $a_{u}, b_{t} \in \mathbb{R}$. Now by the properties of bilinear forms and the moment structure of $\mathcal{M}_{n}$, we have

$$
\begin{aligned}
\langle p, q\rangle_{\mathcal{M}} & =\langle\mathcal{M} \widehat{p}, \widehat{q}\rangle=\left\langle\mathcal{M}\left(\sum_{|u| \leq n} a_{u} \widehat{u}\right), \sum_{|t| \leq n} b_{t} \widehat{t}\right\rangle=\sum_{|u| \leq n|t| \leq n} \sum_{u} b_{t}\langle\mathcal{M} \widehat{u}, \widehat{t}\rangle \\
& =\sum_{|u| \leq n|t| \leq n} \sum_{u} b_{t}\left\langle\mathcal{M} \widehat{t^{*}}, \widehat{u^{*}}\right\rangle=\left\langle\mathcal{M}\left(\sum_{|t| \leq n} b_{t} \widehat{t^{*}}\right), \sum_{|u| \leq n} a_{u} \widehat{u^{*}}\right\rangle=\left\langle\mathcal{M} \widehat{q^{*}}, \widehat{p^{*}}\right\rangle \\
& =\left\langle q^{*}, p^{*}\right\rangle_{\mathcal{M}} .
\end{aligned}
$$

This establishes Proposition 8.3. 
Corollary 8.4. Suppose $\mathcal{M}=\left(\begin{array}{cc}\mathcal{M}_{2} & B_{3} \\ B_{3}^{t} & C_{3}\end{array}\right)$ is a flat extension of a moment matrix $\mathcal{M}_{2}$ with rows and columns indexed by monomials of degree at most 3 . We write $C_{3}=\left(C_{i j}\right)_{i j}$. Then we have:

$$
\begin{array}{llll}
C_{12}=C_{15}, & C_{46}=C_{67}, & C_{22}=C_{55}, & C_{56}, \\
C_{24}=C_{57}, & C_{48}=C_{78}, & C_{28}=C_{58}, & C_{27}=C_{45}, \\
C_{23}=C_{35}, & C_{14}=C_{17}, & C_{44}=C_{77}, & C_{34}=C_{37} .
\end{array}
$$

Therefore assuming $B_{3}$ has a moment structure, $\mathcal{M}$ is a moment matrix of degree 3 if and only if

$$
\begin{array}{llr}
C_{47}=C_{66}, & C_{16}=C_{23}, & C_{28}=C_{44}, \\
C_{25}=C_{33}, & C_{38}=C_{46}, & C_{26}=C_{27}=C_{34} . \\
C_{12}=C_{13}, & C_{48}=C_{68}, & \\
C_{18}=C_{24}, & C_{14}=C_{22}, &
\end{array}
$$

Proof. Claim 1: $C_{12}=C_{15}$.

$$
\begin{aligned}
C_{12}=\left\langle X^{2} Y, X^{3}\right\rangle_{\mathcal{M}} & =\left\langle X^{3}, Y X^{2}\right\rangle_{\mathcal{M}} \quad(\text { by (8.3)) } \\
& =\left\langle Y X^{2}, X^{3}\right\rangle_{\mathcal{M}}=C_{15} \quad(\text { by (8.2)). }
\end{aligned}
$$

Claim 2: $C_{24}=C_{57}$.

$$
\begin{aligned}
C_{24}=\left\langle X Y^{2}, X^{2} Y\right\rangle_{\mathcal{M}} & =\left\langle Y X^{2}, Y^{2} X\right\rangle_{\mathcal{M}} \quad(\text { by }(8.3)) \\
& =\left\langle Y^{2} X, Y X^{2}\right\rangle_{\mathcal{M}}=C_{57} \quad(\text { by (8.2)). }
\end{aligned}
$$

Claim 3: $C_{23}=C_{35}$.

$$
\begin{aligned}
C_{23}=\left\langle X Y X, X^{2} Y\right\rangle_{\mathcal{M}} & =\left\langle Y X^{2}, X Y X\right\rangle_{\mathcal{M}} \quad(\text { by }(8.3)) \\
& =\left\langle X Y X, Y X^{2}\right\rangle_{\mathcal{M}}=C_{35} \quad(\text { by (8.2)). }
\end{aligned}
$$

Claim 4: $C_{46}=C_{67}$.

$$
\begin{aligned}
C_{46}=\left\langle Y X Y, X Y^{2}\right\rangle_{\mathcal{M}} & =\left\langle Y^{2} X, Y X Y\right\rangle_{\mathcal{M}} \quad(\text { by (8.3)) } \\
& =\left\langle Y X Y, Y^{2} X\right\rangle_{\mathcal{M}}=C_{67} \quad(\text { by (8.2)). }
\end{aligned}
$$

Claim 5: $C_{48}=C_{78}$.

$$
\begin{aligned}
C_{48}=\left\langle Y^{3}, X Y^{2}\right\rangle_{\mathcal{M}} & =\left\langle Y^{2} X, Y^{3}\right\rangle_{\mathcal{M}} \quad(\text { by }(8.3)) \\
& =\left\langle Y^{3}, Y^{2} X\right\rangle_{\mathcal{M}}=C_{78} \quad(\text { by (8.2)). }
\end{aligned}
$$

Claim 6: $C_{14}=C_{17}$.

$$
\begin{aligned}
C_{14}=\left\langle X Y^{2}, X^{3}\right\rangle_{\mathcal{M}} & =\left\langle X^{3}, Y^{2} X\right\rangle_{\mathcal{M}} \quad(\text { by }(8.3)) \\
& =\left\langle Y^{2} X, X^{3}\right\rangle_{\mathcal{M}}=C_{17} \quad(\text { by (8.2)). }
\end{aligned}
$$

Claim 7: $C_{22}=C_{55}$.

$$
C_{22}=\left\langle X^{2} Y, X^{2} Y\right\rangle_{\mathcal{M}}=\left\langle Y X^{2}, Y X^{2}\right\rangle_{\mathcal{M}}=C_{55} \quad(\text { by (8.3)). }
$$

Claim 8: $C_{28}=C_{58}$.

$$
\begin{aligned}
C_{28}=\left\langle Y^{3}, X^{2} Y\right\rangle_{\mathcal{M}} & =\left\langle Y X^{2}, Y^{3}\right\rangle_{\mathcal{M}} \quad(\text { by (8.3)) } \\
& =\left\langle Y^{3}, Y X^{2}\right\rangle_{\mathcal{M}}=C_{58} \quad \text { (by (8.2)). }
\end{aligned}
$$

Claim 9: $C_{44}=C_{77}$.

$$
C_{44}=\left\langle X Y^{2}, X Y^{2}\right\rangle_{\mathcal{M}}=\left\langle Y^{2} X, Y^{2} X\right\rangle_{\mathcal{M}}=C_{77} \quad \text { (by (8.3)). }
$$

Claim 10: $C_{26}=C_{56}$.

$$
\begin{aligned}
C_{26}=\left\langle Y X Y, X^{2} Y\right\rangle_{\mathcal{M}} & =\left\langle Y X^{2}, Y X Y\right\rangle_{\mathcal{M}} \quad(\text { by (8.3)) } \\
& =\left\langle Y X Y, Y X^{2}\right\rangle_{\mathcal{M}}=C_{56} \quad(\text { by (8.2)). }
\end{aligned}
$$


Claim 11: $C_{27}=C_{45}$.

$$
\begin{aligned}
C_{27}=\left\langle Y^{2} X, X^{2} Y\right\rangle_{\mathcal{M}} & =\left\langle Y X^{2}, X Y^{2}\right\rangle_{\mathcal{M}} \quad(\text { by }(8.3)) \\
& =\left\langle X Y^{2}, X^{2} Y\right\rangle_{\mathcal{M}}=C_{45} \quad(\text { by }(8.2)) .
\end{aligned}
$$

Claim 12: $C_{34}=C_{37}$.

$$
\begin{aligned}
C_{34}=\left\langle X Y^{2}, X Y X\right\rangle_{\mathcal{M}} & =\left\langle X Y X, Y^{2} X\right\rangle_{\mathcal{M}} \quad(\text { by (8.3)) } \\
& =\left\langle Y^{2} X, X Y X\right\rangle_{\mathcal{M}}=C_{37} \quad \text { (by (8.2)). }
\end{aligned}
$$

This proves the first statement of Corollary 8.4. The second statement follows by observing that if $\mathcal{M}$ is a moment matrix, then the entries of $C_{3}$ are independent from the other entries of $\mathcal{M}$ and combining (8.1) with (8.4).

$$
\begin{aligned}
& \text { If } \mathcal{M}=\left(\begin{array}{cc}
\mathcal{M}_{n} & B_{n+1} \\
B_{n+1}^{t} & C_{n+1}
\end{array}\right) \text { is a flat extension of } \mathcal{M}_{n} \text {, then there is a matrix } W \text { such that } \\
& \qquad B_{n+1}=\mathcal{M}_{n} W \quad \text { and } \quad C_{n+1}=W^{t} \mathcal{M}_{n} W .
\end{aligned}
$$

By the following lemma $C_{n+1}$ is independent of the choice of $W$ satisfying $B_{n+1}=\mathcal{M}_{n} W$.

Lemma 8.5. Let $A \in \mathbb{S R}^{m \times m}$ be a symmetric matrix and $W_{1}, W_{2} \in \mathbb{R}^{m \times p}$ matrices satisfying $A W_{1}=A W_{2}$. Then $W_{1}^{t} A W_{1}=W_{2}^{t} A W_{2}$.

Proof. Since $W_{j}^{t} A W_{j}$ are symmetric matrices, we have

$$
\begin{aligned}
W_{1}^{t} A W_{1}=W_{2}^{t} A W_{2} & \Leftrightarrow\left\langle\left(W_{1}^{t} A W_{1}-W_{2}^{t} A W_{2}\right) v, v\right\rangle \quad \text { for every } v \in \mathbb{R}^{p} \\
& \Leftrightarrow\left\langle A W_{1} v, W_{1} v\right\rangle=\left\langle A W_{2} v, W_{2} v\right\rangle \quad \text { for every } v \in \mathbb{R}^{p} .
\end{aligned}
$$

Let us write $v_{1}:=W_{1} v$ and $v_{2}:=W_{2} v$. By assumption $A W_{1}=A W_{2}$ it follows that $A v_{1}=A v_{2}$. The following calculation holds:

$$
\begin{aligned}
0 & =\left\langle A\left(v_{1}-v_{2}\right),\left(v_{1}+v_{2}\right)\right\rangle=\left\langle A v_{1}, v_{1}\right\rangle+\left\langle A v_{1}, v_{2}\right\rangle-\left\langle A v_{2}, v_{1}\right\rangle-\left\langle A v_{2}, v_{2}\right\rangle \\
& =\left\langle A v_{1}, v_{1}\right\rangle+\left\langle v_{1}, A v_{2}\right\rangle-\left\langle A v_{2}, v_{1}\right\rangle-\left\langle A v_{2}, v_{2}\right\rangle \\
& =\left\langle A v_{1}, v_{1}\right\rangle+\left\langle A v_{2}, v_{1}\right\rangle-\left\langle A v_{2}, v_{1}\right\rangle-\left\langle A v_{2}, v_{2}\right\rangle=\left\langle A v_{1}, v_{1}\right\rangle-\left\langle A v_{2}, v_{2}\right\rangle .
\end{aligned}
$$

This concludes the proof of the lemma.

8.2. Relation $\mathbb{Y}^{2}=\mathbb{1}-\mathbb{X}^{2}$. The candidate for $B_{3}$ in a moment matrix $\mathcal{M}_{3}$ generated by the measure for $\mathcal{M}_{2}$ is given by the following.

Proposition 8.6. Let $\beta \equiv \beta^{(4)}$ be a nc sequence with a moment matrix $\mathcal{M}_{2}$ of rank 6 satisfying the relation $\mathbb{Y}^{2}=$ $\mathbb{1}-\mathbb{X}^{2}$. Suppose $\beta$ admits a nc measure $\mu$. If $\mathcal{M}_{3}=\left(\begin{array}{ll}\mathcal{M}_{2} & B_{3} \\ B_{3}^{t} & C_{3}\end{array}\right)$ is a moment matrix generated by the measure $\mu$, then $B_{3}$ satisfies

$$
\begin{array}{r}
\beta_{X^{2} Y^{3}}=\beta_{X Y^{2} X Y}=\beta_{X^{2} Y}-q, \\
\beta_{X^{3} Y^{2}}=\beta_{X^{2} Y X Y}=\beta_{X^{3}}-p, \\
\beta_{X Y^{4}}=\beta_{X}-2 \beta_{X^{3}}+p,
\end{array}
$$

$$
\begin{array}{r}
\beta_{Y^{5}}=\beta_{Y}-2 \beta_{X^{2} Y}+q, \\
\beta_{X^{5}}=p, \\
\beta_{X^{4} Y}=q,
\end{array}
$$

where $p, q \in \mathbb{R}$ are parameters.

Proof. The RG relations which must hold in $\mathcal{M}_{3}$ are

$$
\begin{aligned}
& \mathbb{Y}^{3}=\mathbb{Y}-\mathbb{X}^{2} \mathbb{Y}, \\
& \mathbb{Y}^{3}=\mathbb{Y}-\mathbb{Y}^{2},
\end{aligned}
$$

$$
\begin{aligned}
& \mathbb{X} \mathbb{Y}^{2}=\mathbb{X}-\mathbb{X}^{3} \\
& \mathbb{Y}^{2} \mathbb{X}=\mathbb{X}-\mathbb{X}^{3}
\end{aligned}
$$

From these relations we get the following system: 


$$
\begin{aligned}
\beta_{X Y^{4}}=\left(\beta_{X}-\beta_{X^{3}}\right)-\beta_{X^{3} Y^{2}}, & \beta_{X^{2} Y^{3}} & =\beta_{X^{2} Y}-\beta_{X^{4} Y}, \\
\beta_{X Y^{4}}=\left(\beta_{X}-\beta_{X^{3}}\right)-\beta_{X^{2} Y X Y}, & \beta_{X^{3} Y^{2}}= & \beta_{X^{3}}-\beta_{X^{5}}, \\
\beta_{Y^{5}}=\left(\beta_{Y}-\beta_{X^{2} Y}\right)-\beta_{X^{2} Y^{3}}, & \beta_{X Y^{2} X Y}= & \beta_{X^{2} Y}-\beta_{X^{4} Y} .
\end{aligned}
$$

Now the solution of this system is given by the statement of the proposition.

Theorem 8.7. Suppose $\beta \equiv \beta^{(4)}$ is a nc sequence with a moment matrix $\mathcal{M}_{2}$ of rank 6 satisfying the relation $\mathbb{Y}^{2}=$ $\mathbb{1}-\mathbb{X}^{2}$. Let us define the moments of degree 5 by (8.19) and $B_{3}$ as in Proposition 8.1. Then the following are true:

(1) There exists a matrix $W \in \mathbb{R}^{7 \times 10}$ such that

$$
B_{3}=\mathcal{M}_{2} W .
$$

(2) We write $M_{1}=\left\{\mathbb{1}, \mathbb{X}, \mathbb{Y}, \mathbb{X}^{2}, \mathbb{X} \mathbb{Y}, \mathbb{Y} \mathbb{X}\right\}$ and $M_{2}=\left\{\mathbb{X}^{3}, \mathbb{X}^{2} \mathbb{Y}, \mathbb{X} \mathbb{Y}, \mathbb{X} \mathbb{Y}^{2}, \mathbb{Y}^{2}, \mathbb{Y} \mathbb{X}, \mathbb{Y}^{2} \mathbb{X}, \mathbb{Y}^{3}\right\}$. Let $W_{1} \in$ $\mathbb{R}^{6} \times \mathbb{R}^{10}$ be the matrix

$$
W_{1}=\left.\left(\left.\mathcal{M}_{2}\right|_{M_{1}}\right)^{-1} B_{3}\right|_{M_{1}, M_{2}}
$$

If $\mathcal{M}=\left(\begin{array}{ll}\mathcal{M}_{2} & B_{3} \\ B_{3}^{t} & C_{3}\end{array}\right)$ is a flat extension of $\mathcal{M}_{2}$, then $C_{3}=\left(C_{i j}\right)_{i j}$ is equal to $\left.W_{1}^{t} \mathcal{M}_{2}\right|_{M_{1}} W_{1}$ and $\mathcal{M}$ has a moment structure if and only if

$$
\begin{array}{lll}
C_{47}=C_{66}, & C_{16}=C_{23}, & C_{28}=C_{44}, \\
C_{25}=C_{33}, & C_{48}=C_{68}, & C_{26}=C_{27} . \\
C_{12}=C_{13}, & C_{14}=C_{22}, &
\end{array}
$$

Proof. To prove (1) we have to show that every column of $B_{3}$ belongs to the linear span of the columns of $\mathcal{M}_{2}$. Since the proofs are analogous, we will establish this only for the column $\mathbb{X}^{3}$. Since $\left.\mathcal{M}_{2}\right|_{M_{1}}$ is positive definite, it follows that

$$
\left.\mathbb{X}^{3}\right|_{M_{1}}=\left.a_{1} \mathbb{1}\right|_{M_{1}}+\left.a_{2} \mathbb{X}\right|_{M_{1}}+\left.a_{3} \mathbb{Y}\right|_{M_{1}}+\left.a_{4} \mathbb{X}^{2}\right|_{M_{1}}+\left.a_{5} \mathbb{X} \mathbb{Y}\right|_{M_{1}}+\left.a_{6} \mathbb{Y} \mathbb{X}\right|_{M_{1}},
$$

for some $a_{i} \in \mathbb{R}$ where $\left.*\right|_{M_{1}}$ denotes the restriction of the column $*$ to the rows from $M_{1}$. Notice that $a_{5}=a_{6}$. Using the relation $\mathbb{Y}^{2}=\mathbb{1}-\mathbb{X}^{2}$ we calculate

$$
\begin{array}{r}
a_{1} \beta_{Y^{2}}+a_{2} \beta_{X Y^{2}}+a_{3} \beta_{Y^{3}}+a_{4} \beta_{X^{2} Y 2}+2 a_{5} \beta_{X Y^{3}} \\
=a_{1}\left(\beta_{1}-\beta_{X^{2}}\right)+a_{2}\left(\beta_{X}-\beta_{X^{3}}\right)+a_{3}\left(\beta_{Y}-\beta_{X^{2} Y}\right)+a_{4}\left(\beta_{X^{2}}-\beta_{X^{4}}\right) \\
+2 a_{5}\left(\beta_{X Y}-\beta_{X^{3} Y}\right) \\
=\beta_{X^{3}}-p .
\end{array}
$$

By the form of $B_{3}$ it follows that

$$
\mathbb{X}^{3}=a_{1} \mathbb{1}+a_{2} \mathbb{X}+a_{3} \mathbb{Y}+a_{4} \mathbb{X}^{2}+a_{5} \mathbb{X} \mathbb{Y}+a_{6} \mathbb{Y} \mathbb{X} \text { in } \mathcal{M}_{3}
$$

This proves part (1).

If $\mathcal{M}$ is a flat extension of $\mathcal{M}_{2}$, then in particular $\operatorname{rank}\left(\left(\begin{array}{ll}\mathcal{M}_{2} & B_{3}\end{array}\right)\right)=\operatorname{rank} \mathcal{M}_{2}$. Since the columns from $M_{1}$ are the basis for the column space of $\mathcal{M}_{2}$, we have

$$
B_{3}=\mathcal{M}_{2}\left(\begin{array}{c}
W_{1} \\
0_{1 \times 10}
\end{array}\right) .
$$

By Lemma 8.5 it follows that

$$
C_{3}=\left(\begin{array}{ll}
W_{1}^{t} & 0_{10 \times 1}
\end{array}\right) \mathcal{M}_{2}\left(\begin{array}{c}
W_{1} \\
0_{1 \times 10}
\end{array}\right)=\left.W_{1}^{t} \mathcal{M}_{2}\right|_{M_{1}} W_{1} .
$$

Now we will establish the relations which will prove that the system (8.5) holds if and only if the system (8.22) holds.

Claim 1: $C_{18}=C_{24}$. 


$$
\begin{aligned}
C_{18}=\left\langle Y^{3}, X^{3}\right\rangle_{\mathcal{M}_{3}} & =\left\langle Y-X^{2} Y, X^{3}\right\rangle_{\mathcal{M}_{3}} \quad \text { (by RG relations) } \\
& =\left\langle Y, X^{3}\right\rangle_{\mathcal{M}_{3}}-\left\langle X^{3}, X^{2} Y\right\rangle_{\mathcal{M}_{3}} \quad \text { (by (8.2)) } \\
& \left.=\left\langle X, X^{2} Y\right\rangle_{\mathcal{M}_{3}}-\left\langle X^{3}, X^{2} Y\right\rangle_{\mathcal{M}_{3}} \quad \text { (by the moment structure of } B_{3}\right) \\
& =\left\langle X Y^{2}, X^{2} Y\right\rangle_{\mathcal{M}_{3}}=C_{24} \quad \text { (by RG relations). }
\end{aligned}
$$

Claim 2: $C_{16}-C_{23}=C_{38}-C_{46}$.

$$
\begin{aligned}
C_{16}-C_{23}= & \left\langle Y X Y, X^{3}\right\rangle_{\mathcal{M}_{3}}-\left\langle X Y X, X^{2} Y\right\rangle_{\mathcal{M}_{3}} \\
= & \left\langle Y X Y, X-X Y^{2}\right\rangle_{\mathcal{M}_{3}}-\left\langle X^{2} Y, X Y X\right\rangle_{\mathcal{M}_{3}} \quad \text { (by RG relations and (8.2)) } \\
= & \left(\langle X, Y X Y\rangle_{\mathcal{M}_{3}}-\left\langle X^{2} Y, X Y X\right\rangle_{\mathcal{M}_{3}}\right)-\left\langle Y X Y, X Y^{2}\right\rangle_{\mathcal{M}_{3}} \\
& \left.\quad \text { (by the moment structure of } B_{3}\right) \\
= & \left\langle Y^{3}, X Y X\right\rangle_{\mathcal{M}_{3}}-\left\langle Y X Y, X Y^{2}\right\rangle_{\mathcal{M}_{3}} \quad \text { (by RG relations) } \\
= & C_{38}-C_{46} .
\end{aligned}
$$

Claim 3: $C_{37}-C_{27}=C_{12}-C_{13}$.

$$
\begin{aligned}
C_{37}-C_{27}= & \left\langle Y^{2} X, X Y X\right\rangle_{\mathcal{M}_{3}}-\left\langle Y^{2} X, X^{2} Y\right\rangle_{\mathcal{M}_{3}} \\
= & \left\langle X-X^{3}, X Y X\right\rangle_{\mathcal{M}_{3}}-\left\langle Y^{2} X, X^{2} Y\right\rangle_{\mathcal{M}_{3}} \quad \text { (by RG relations) } \\
= & \left(\left\langle X, X^{2} Y\right\rangle_{\mathcal{M}_{3}}-\left\langle Y^{2} X, X^{2} Y\right\rangle_{\mathcal{M}_{3}}\right)-\left\langle X^{3}, X Y X\right\rangle_{\mathcal{M}_{3}} \\
& \left.\quad \quad \text { by the moment structure of } B_{3}\right) \\
= & \left\langle X^{3}, X^{2} Y\right\rangle_{\mathcal{M}_{3}}-\left\langle X^{3}, X Y X\right\rangle_{\mathcal{M}_{3}} \\
= & C_{12}-C_{13} .
\end{aligned}
$$

Using Claims 1-3 proves Theorem 8.7 (2).

Remark 8.8. Assume the notation as in Theorem 8.7. If $\mathcal{M}$ is a flat extension and has a moment structure, then we must also have

which follows by the following:

$$
C_{26}=C_{18} \quad \text { and } \quad C_{14}=C_{25},
$$

and

$$
\begin{aligned}
C_{48}-C_{68}= & \left\langle Y^{3}, X Y^{2}\right\rangle_{\mathcal{M}_{3}}-\left\langle Y^{3}, Y X Y\right\rangle_{\mathcal{M}_{3}} \\
= & \left\langle Y^{3}, X Y^{2}\right\rangle_{\mathcal{M}_{3}}-\left\langle Y-X^{2} Y, Y X Y\right\rangle_{\mathcal{M}_{3}} \quad \text { (by RG relations) } \\
= & \left\langle Y^{3}, X Y^{2}\right\rangle_{\mathcal{M}_{3}}-\left\langle Y^{3}, X\right\rangle_{\mathcal{M}_{3}}+\left\langle X^{2} Y, Y X Y\right\rangle_{\mathcal{M}_{3}} \\
& \left.\quad \text { (by the moment structure of } B_{3}\right) \\
= & \left\langle X^{2} Y, Y X Y\right\rangle_{\mathcal{M}_{3}}-\left\langle Y^{3}, X^{3}\right\rangle_{\mathcal{M}_{3}} \quad \text { (by RG relations) } \\
= & \left\langle Y X Y, X^{2} Y\right\rangle_{\mathcal{M}_{3}}-\left\langle X^{3}, Y^{3}\right\rangle_{\mathcal{M}_{3}} \quad \text { (by (8.2)) } \\
= & C_{26}-C_{18},
\end{aligned}
$$

$$
\begin{aligned}
C_{58}-C_{44} & =\left\langle Y^{3}, Y X^{2}\right\rangle_{\mathcal{M}_{3}}-\left\langle X Y^{2}, X Y^{2}\right\rangle_{\mathcal{M}_{3}} \\
& =\left\langle Y-X^{2} Y, Y X^{2}\right\rangle_{\mathcal{M}_{3}}-\left\langle X Y^{2}, X Y^{2}\right\rangle_{\mathcal{M}_{3}} \quad \text { (by RG relations) } \\
& =\left(\left\langle Y, Y X^{2}\right\rangle_{\mathcal{M}_{3}}-\left\langle X Y^{2}, X Y^{2}\right\rangle_{\mathcal{M}_{3}}\right)-\left\langle Y X^{2}, X^{2} Y\right\rangle_{\mathcal{M}_{3}} \quad \text { (by (8.2)) } \\
& =\left(\left\langle X, X Y^{2}\right\rangle_{\mathcal{M}_{3}}-\left\langle X Y^{2}, X Y^{2}\right\rangle_{\mathcal{M}_{3}}\right)-\left\langle Y X^{2}, X^{2} Y\right\rangle_{\mathcal{M}_{3}}
\end{aligned}
$$

(by the moment structure of $B_{3}$ )

$$
\begin{aligned}
& =\left\langle X Y^{2}, X^{3}\right\rangle_{\mathcal{M}_{3}}-\left\langle Y X^{2}, X^{2} Y\right\rangle_{\mathcal{M}_{3}} \quad \text { (by RG relations and (8.2)) } \\
& =C_{14}-C_{25} .
\end{aligned}
$$

We present now a special case which highlights the difference between the classical commutative and the tracial bivariate quartic moment problems with a moment matrix $\mathcal{M}_{2}$ satisfying exactly $\mathbb{Y}^{2}=\mathbb{1}-\mathbb{X}^{2}$. By Theorem 2.7 (3), 
in the $\mathrm{cm}$ case $\mathcal{M}_{2}$ always admits a flat extension to the moment matrix $\mathcal{M}_{3}$. The following example shows that this is not true in the nc case. However, a nc measure in this example still exists.

Example 8.9. For $\beta_{X^{4}} \in\left(\frac{1}{4}, \frac{1}{2}\right)$, the following matrices are psd moment matrices of rank 6 satisfying the relation $\mathbb{Y}^{2}=\mathbb{1}-\mathbb{X}^{2}$,

$$
\mathcal{M}_{2}\left(\beta_{X^{4}}\right)=\left(\begin{array}{ccccccc}
1 & 0 & 0 & \frac{1}{2} & 0 & 0 & \frac{1}{2} \\
0 & \frac{1}{2} & 0 & 0 & 0 & 0 & 0 \\
0 & 0 & \frac{1}{2} & 0 & 0 & 0 & 0 \\
\frac{1}{2} & 0 & 0 & \beta_{X^{4}} & 0 & 0 & \frac{1}{2}-\beta_{X^{4}} \\
0 & 0 & 0 & 0 & \frac{1}{2}-\beta_{X^{4}} & 0 & 0 \\
0 & 0 & 0 & 0 & 0 & \frac{1}{2}-\beta_{X^{4}} & 0 \\
\frac{1}{2} & 0 & 0 & \frac{1}{2}-\beta_{X^{4}} & 0 & 0 & \beta_{X^{4}}
\end{array}\right) .
$$

Let us define the moments of degree 5 by (8.19), $B_{3}$ as in Proposition 8.1 and $M_{1}, M_{2}, C_{3}$ as in Theorem 8.7 (2). It is easy to check that

$$
\left(\left.\mathcal{M}_{2}\right|_{M_{1}}\right)^{-1}=\left(\begin{array}{cccc}
4 \beta_{X^{4}} & 0 & 0 & \frac{2}{1-4 \beta_{X^{4}}} \\
0 & 2 & 0 & 0 \\
0 & 0 & 2 & 0 \\
\frac{2}{1-4 \beta_{X^{4}}} & 0 & 0 & \frac{4}{4 \beta_{X^{4}}-1}
\end{array}\right) \bigoplus\left(\begin{array}{cc}
\frac{2}{1-2 \beta_{X^{4}}} & 0 \\
0 & \frac{2}{1-2 \beta_{X^{4}}}
\end{array}\right) .
$$

By a straightworward calculation of $\left(\left.B_{3}\right|_{M_{1}, M_{2}}\right)^{t}\left(\left.\mathcal{M}_{2}\right|_{M_{1}}\right)^{-1}\left(\left.B_{3}\right|_{M_{1}, M_{2}}\right)$ we get that

$$
\begin{aligned}
& C_{47}\left(p, q, \beta_{X^{4}}\right)=\frac{1}{2}-2 \beta_{X^{4}}+2 \beta_{X^{4}}^{2}+\frac{4 q^{2}}{1-2 \beta_{X^{4}}}+\frac{4 p^{2}}{-1+4 \beta_{X^{4}}}, \\
& C_{66}\left(p, q, \beta_{X^{4}}\right)=\frac{4 q^{2}}{1-2 \beta_{X^{4}}}+\frac{4 p^{2}}{-1+4 \beta_{X^{4}}},
\end{aligned}
$$

and hence

$$
\left(C_{47}-C_{66}\right)\left(p, q, \beta_{X^{4}}\right)=\frac{1}{2}\left(1-2 \beta_{X^{4}}\right)^{2} \neq 0 .
$$

Therefore the system from Theorem 8.7 does not have a solution and $\mathcal{M}_{2}\left(\beta_{X^{4}}\right)$ does not admit a flat extension with a moment structure $\mathcal{M}_{3}\left(\beta_{X^{4}}\right)$. However, for every $\beta_{X^{4}} \in\left(\frac{1}{4}, \frac{1}{2}\right), \mathcal{M}_{2}\left(\beta_{X^{4}}\right)$ admits a nc measure by Theorem 7.5 (1).

8.3. Relation $\mathbb{X} \mathbb{Y}+\mathbb{Y} \mathbb{X}=\mathbf{0}$. The candidate for $B_{3}$ in a moment matrix $\mathcal{M}_{3}$ generated by the nc measure for $\mathcal{M}_{2}$ is given by the following.

Proposition 8.10. Let $\beta \equiv \beta^{(4)}$ be a sequence with a moment matrix $\mathcal{M}_{2}$ of rank 6 satisfying the relation $\mathbb{X} \mathbb{Y}+\mathbb{Y} \mathbb{X}=$ 0. Suppose $\beta$ admits a nc measure $\mu$. If $\mathcal{M}_{3}=\left(\begin{array}{ll}\mathcal{M}_{2} & B_{3} \\ B_{3}^{t} & C_{3}\end{array}\right)$ is a moment matrix generated by the nc measure $\mu$, then $B_{3}$ satisfies

$$
\begin{array}{r}
\beta_{X^{4} Y}=\beta_{X^{2} Y X Y}=\beta_{X^{3} Y^{2}}=\beta_{X^{2} Y^{3}}=\beta_{X Y^{2} X Y}=\beta_{X Y^{4}}=0, \\
\beta_{X^{5}}=p \quad \text { and } \quad \beta_{Y^{5}}=q,
\end{array}
$$

where $p, q \in \mathbb{R}$ are parameters.

Proof. The RG relations which must hold in $\mathcal{M}_{3}$ are

$$
\begin{aligned}
& \mathbb{X}^{2} \mathbb{Y}+\mathbb{X} \mathbb{Y} \mathbb{X}= \\
& \mathbb{X} \mathbb{Y}+\mathbb{Y}^{2}=\mathbf{0}
\end{aligned}
$$

$$
\begin{aligned}
\mathbb{Y} \mathbb{Y}+\mathbb{Y}^{2} \mathbb{X} & =\mathbf{0} \\
\mathbb{X} \mathbb{Y}^{2}+\mathbb{Y} \mathbb{Y} & =\mathbf{0}
\end{aligned}
$$

From this relations we get the following system:

$$
\begin{aligned}
2 \beta_{X^{4} Y} & =0, & \beta_{X^{2} Y^{3}}+\beta_{X Y^{2} X Y} & =0 \\
\beta_{X^{3} Y^{2}}+\beta_{X^{2} Y X Y} & =0, & 2 \beta_{X Y^{2} X Y} & =0 \\
2 \beta_{X^{2} Y X Y} & =0, & 2 \beta_{X Y^{4}} & =0
\end{aligned}
$$

Now the solution of this system is given by the statement of the proposition. 
Theorem 8.11. Suppose $\beta \equiv \beta^{(4)}$ is a nc sequence with a moment matrix $\mathcal{M}_{2}$ of rank 6 satisfying the relation $\mathbb{X} \mathbb{Y}+\mathbb{Y} \mathbb{X}=0$. Let us define the moments of degree 5 by (8.50) and $B_{3}$ as in Proposition 8.1. Then the following are true:

(1) There exists a matrix $W \in \mathbb{R}^{7 \times 10}$ satisfying

$$
B_{3}=\mathcal{M}_{2} W
$$

(2) We write $M_{1}=\left\{\mathbb{1}, \mathbb{X}, \mathbb{Y}, \mathbb{X}^{2}, \mathbb{X} \mathbb{Y}, \mathbb{Y}^{2}\right\}$ and $M_{2}=\left\{\mathbb{X}^{3}, \mathbb{X}^{2} \mathbb{Y}, \mathbb{X} \mathbb{Y}, \mathbb{X} \mathbb{Y}^{2}, \mathbb{Y} \mathbb{X}^{2}, \mathbb{Y} \mathbb{Y}, \mathbb{Y} \mathbb{X}^{2}, \mathbb{Y}^{3}\right\}$. Let $W_{1} \in$ $\mathbb{R}^{6 \times 10}$ be the matrix

$$
\begin{aligned}
& W_{1}=\left.\left(\left.\mathcal{M}_{2}\right|_{M_{1}}\right)^{-1} B_{3}\right|_{M_{1}, M_{2}} . \\
& \text { If } \mathcal{M}=\left(\begin{array}{ll}
\mathcal{M}_{2} & B_{3} \\
B_{3}^{t} & C_{3}
\end{array}\right) \text { is a flat extension of } \mathcal{M}_{2} \text {, then } C_{3}=\left(C_{i j}\right)_{i j} \text { is equal to }\left.W_{1}^{t} \mathcal{M}_{2}\right|_{M_{1}} W_{1} \text { and } \mathcal{M} \text { has a } \\
& C_{12}=C_{13}=0 \\
& C_{38}=C_{46}, \\
& C_{28}=C_{44} \text {, } \\
& C_{18}=C_{24} \\
& C_{16}=C_{23} \text {, } \\
& \begin{array}{r}
C_{48}=C_{68}=0 \\
C_{14}=C_{22}
\end{array} \\
& C_{26}=C_{27}=C_{34}
\end{aligned}
$$

Proof. The proof is analogous to the proof of Theorem 8.7. Using similar arguments we prove the following claim:

Claim: $C_{47}=C_{66}, C_{25}=C_{33}, C_{12}=-C_{13}, C_{24}=C_{57}, C_{48}=-C_{68}$.

By Claim the system (8.5) holds if and only if the system (8.52) holds.

We present now a special case which shows that for a nc sequence $\beta$ with a psd moment matrix $\mathcal{M}_{2}$ satisfying exactly $\mathbb{X} \mathbb{Y}+\mathbb{Y} \mathbb{X}=\mathbf{0}$, admitting a flat extension to the moment matrix $\mathcal{M}_{3}$ is not equivalent to admitting a nc measure.

Example 8.12. For $\beta_{X^{4}}>1$, the following matrices are psd moment matrices of rank 6 satisfying the relation $\mathbb{X} \mathbb{Y}+$ $\mathbb{Y} \mathbb{X}=\mathbf{0}$,

$$
\mathcal{M}_{2}\left(\beta_{X^{4}}\right)=\left(\begin{array}{ccccccc}
1 & 0 & 0 & 1 & 0 & 0 & 1 \\
0 & 1 & 0 & 0 & 0 & 0 & 0 \\
0 & 0 & 1 & 0 & 0 & 0 & 0 \\
1 & 0 & 0 & \beta_{X^{4}} & 0 & 0 & 1 \\
0 & 0 & 0 & 0 & 1 & -1 & 0 \\
0 & 0 & 0 & 0 & -1 & 1 & 0 \\
1 & 0 & 0 & 1 & 0 & 0 & 2
\end{array}\right) .
$$

Let us define the moments of degree 5 by (8.50), $B_{3}$ as in Proposition 8.1 and $M_{1}, M_{2}, C_{3}$ as in Theorem 8.11 (2). It is easy to check that

$$
\left(\mathcal{M}_{2} \mid M_{1}\right)^{-1}=\left(\begin{array}{cccccc}
\frac{2 \beta_{X^{4}}-1}{\beta_{X^{4}}-1} & 0 & 0 & \frac{1}{1-\beta_{X^{4}}} & 0 & -1 \\
0 & 1 & 0 & 0 & 0 & 0 \\
0 & 0 & 1 & 0 & 0 & 0 \\
\frac{1}{1-\beta_{X^{4}}} & 0 & 0 & \frac{1}{\beta_{X^{4}}-1} & 0 & 0 \\
0 & 0 & 0 & 0 & 1 & 0 \\
-1 & 0 & 0 & 0 & 0 & 1
\end{array}\right) .
$$

By a straightworward calculation of $\left(\left.B_{3}\right|_{M_{1}, M_{2}}\right)^{t}\left(\left.\mathcal{M}_{2}\right|_{M_{1}}\right)^{-1}\left(\left.B_{3}\right|_{M_{1}, M_{2}}\right)$ we get that

$$
C_{3}(p, q)=\left(\begin{array}{cccccccc}
\frac{p^{2}}{\beta_{X^{4}}-1}+4 & 0 & 0 & 2 & 0 & -2 & 2 & 0 \\
0 & 1 & -1 & 0 & 1 & 0 & 0 & 2 \\
0 & -1 & 1 & 0 & -1 & 0 & 0 & -2 \\
2 & 0 & 0 & 1 & 0 & -1 & 1 & 0 \\
0 & 1 & -1 & 0 & 1 & 0 & 0 & 2 \\
-2 & 0 & 0 & -1 & 0 & 1 & -1 & 0 \\
2 & 0 & 0 & 1 & 0 & -1 & 1 & 0 \\
0 & 2 & -2 & 0 & 2 & 0 & 0 & q^{2}+4 .
\end{array}\right)
$$

The system (8.52) does not have a solution, e.g., $-2=C_{16} \neq C_{23}=-1$. Hence $\mathcal{M}_{2}\left(\beta_{X^{4}}\right)$ does not admit a flat extension with a moment structure $\mathcal{M}_{3}\left(\beta_{X^{4}}\right)$. However, for every $\beta_{X^{4}}>1, \mathcal{M}_{2}\left(\beta_{X^{4}}\right)$ admits a nc measure by Theorem 7.8 (1). 
8.4. Relation $\mathbb{Y}^{2}=\mathbb{1}+\mathbb{X}^{2}$. The form of $\mathcal{M}_{2}$ is given by the following proposition.

Proposition 8.13. Let $\beta \equiv \beta^{(4)}$ be a nc sequence with a moment matrix $\mathcal{M}_{2}$ satisfying the relation

$$
\mathbb{Y}^{2}=\mathbb{1}+\mathbb{X}^{2}
$$

Then $\mathcal{M}_{2}$ if of the form

$$
\left(\begin{array}{cccc}
\beta_{1} & \beta_{X} & \beta_{Y} & \beta_{X^{2}} \\
\beta_{X} & \beta_{X^{2}} & \beta_{X Y} & \beta_{X^{3}} \\
\beta_{Y} & \beta_{X Y} & \beta_{1}+\beta_{X^{2}} & \beta_{X^{2} Y} \\
\beta_{X^{2}} & \beta_{X^{3}} & \beta_{X^{2} Y} & \beta_{X^{4}} \\
\beta_{X Y} & \beta_{X^{2} Y} & \beta_{X}+\beta_{X^{3}} & \beta_{X^{3} Y} \\
\beta_{X Y} & \beta_{X^{2} Y} & \beta_{X}+\beta_{X^{3}} & \beta_{X^{3} Y} \\
\beta_{1}+\beta_{X^{2}} & \beta_{X}+\beta_{X^{3}} & \beta_{Y}+\beta_{X^{2} Y} & \beta_{X^{2}}+\beta_{X^{4}}
\end{array}\right.
$$

$\beta_{X Y}$
$\beta_{X^{2} Y}$
$\beta_{X}+\beta_{X^{3}}$
$\beta_{X^{3} Y}$
$\beta_{X^{2}+\beta_{X^{4}}}$
$\beta_{X Y X Y}$
$\beta_{X Y}+\beta_{X^{3} Y}$

$\beta_{X Y}$
$\beta_{X^{2} Y}$
$\beta_{X}+\beta_{X^{3}}$
$\beta_{X^{3} Y}$
$\beta_{X Y X Y}$
$\beta_{X^{2}}+\beta_{X^{4}}$
$\beta_{X Y}+\beta_{X^{3} Y}$

$$
\left.\begin{array}{c}
\beta_{1}+\beta_{X^{2}} \\
\beta_{X}+\beta_{X^{3}} \\
\beta_{Y}+\beta_{X^{2} Y} \\
\beta_{X^{2}}+\beta_{X^{4}} \\
\beta_{X Y}+\beta_{X^{3} Y} \\
\beta_{X Y}+\beta_{X^{3} Y} \\
\beta_{1}+2 \beta_{X^{2}}+\beta_{X^{4}}
\end{array}\right)
$$

Proof. The relation (8.53) gives us the following system in $\mathcal{M}_{2}$

$$
\begin{array}{cr}
\beta_{Y^{2}}=\beta_{1}+\beta_{X^{2}}, & \beta_{X^{2} Y^{2}}=\beta_{X^{2}}+\beta_{X^{4}}, \\
\beta_{X Y^{2}}=\beta_{X}+\beta_{X^{3}}, & \beta_{X Y^{3}}=\beta_{X Y}+\beta_{X^{3} Y}, \\
\beta_{Y^{3}}=\beta_{Y}+\beta_{X^{2} Y}, & \beta_{Y^{4}}=\beta_{Y^{2}}+\beta_{X^{2} Y^{2}} .
\end{array}
$$

Plugging in the expressions for $\beta_{Y^{2}}$ and $\beta_{X^{2} Y^{2}}$ in the expression for $\beta_{Y^{4}}$ gives the form (8.54) of $\mathcal{M}_{2}$.

The candidate for $B_{3}$ in a moment matrix $\mathcal{M}_{3}$ generated by the measure for $\mathcal{M}_{2}$ is given by the following.

Proposition 8.14. Let $\beta \equiv \beta^{(4)}$ be a nc sequence with a moment matrix $\mathcal{M}_{2}$ of rank 6 satisfying the relation $\mathbb{Y}^{2}=$ $\mathbb{1}+\mathbb{X}^{2}$. Suppose $\beta$ admits a nc measure $\mu$. If $\mathcal{M}_{3}=\left(\begin{array}{cc}\mathcal{M}_{2} & B_{3} \\ B_{3}^{t} & C_{3}\end{array}\right)$ is a moment matrix generated by the measure $\mu$, then $B_{3}$ satisfies

$$
\begin{array}{r}
\beta_{X^{2} Y^{3}}=\beta_{X Y^{2} X Y}=\beta_{X^{2} Y}+q, \\
\beta_{X^{3} Y^{2}}=\beta_{X^{2} Y X Y}=\beta_{X^{3}}+p, \\
\beta_{X Y^{4}}=\beta_{X}+2 \beta_{X^{3}}+p,
\end{array}
$$

$$
\begin{array}{r}
\beta_{Y^{5}}=\beta_{Y}+2 \beta_{X^{2} Y}+q, \\
\beta_{X^{5}}=p, \\
\beta_{X^{4} Y}=q,
\end{array}
$$

where $p, q \in \mathbb{R}$ are parameters.

Proof. The RG relations which must hold in $\mathcal{M}_{3}$ are

$$
\begin{aligned}
& \mathbb{Y}^{3}=\mathbb{Y}+\mathbb{X}^{2} \mathbb{Y} \\
& \mathbb{Y}^{3}=\mathbb{Y}+\mathbb{Y}^{2}
\end{aligned}
$$

$$
\begin{aligned}
& \mathbb{X} \mathbb{Y}^{2}=\mathbb{X}+\mathbb{X}^{3} \\
& \mathbb{Y}^{2} \mathbb{X}=\mathbb{X}+\mathbb{X}^{3}
\end{aligned}
$$

From these relations we get the following system:

$$
\begin{aligned}
\beta_{X Y^{4}} & =\left(\beta_{X}+\beta_{X^{3}}\right)+\beta_{X^{3} Y^{2}}, & \beta_{X^{2} Y^{3}} & =\beta_{X^{2} Y}+\beta_{X^{4} Y}, \\
\beta_{X Y^{4}} & =\left(\beta_{X}+\beta_{X^{3}}\right)+\beta_{X^{2} Y X Y}, & \beta_{X^{3} Y^{2}} & =\beta_{X^{3}}+\beta_{X^{5}}, \\
\beta_{Y^{5}} & =\left(\beta_{Y}+\beta_{X^{2} Y}\right)+\beta_{X^{2} Y^{3}}, & \beta_{X Y^{2} X Y} & =\beta_{X^{2} Y}+\beta_{X^{4} Y} .
\end{aligned}
$$

The solution of this system is given by the statement of the proposition.

Theorem 8.15. Suppose $\beta \equiv \beta^{(4)}$ is a nc sequence with a moment matrix $\mathcal{M}_{2}$ of rank 6 satisfying the relation $\mathbb{Y}^{2}=\mathbb{1}+\mathbb{X}^{2}$. Let us define the moments of degree 5 by (8.56) and $B_{3}$ as in Proposition 8.1. Then the following are true:

(1) There exists a matrix $W \in \mathbb{R}^{7 \times 10}$ satisfying

$$
B_{3}=\mathcal{M}_{2} W
$$


(2) We write $M_{1}=\left\{\mathbb{1}, \mathbb{X}, \mathbb{Y}, \mathbb{X}^{2}, \mathbb{X} \mathbb{Y}, \mathbb{Y}\right\}$ and $M_{2}=\left\{\mathbb{X}^{3}, \mathbb{X}^{2} \mathbb{Y}, \mathbb{X} \mathbb{Y}, \mathbb{X} \mathbb{Y}^{2}, \mathbb{Y} \mathbb{X}^{2}, \mathbb{Y} \mathbb{X}, \mathbb{Y}^{2} \mathbb{X}, \mathbb{Y}^{3}\right\}$. Let $W_{1} \in$ $\mathbb{R}^{6 \times 10}$ be the matrix

$$
\begin{aligned}
& W_{1}=\left.\left(\left.\mathcal{M}_{2}\right|_{M_{1}}\right)^{-1} B_{3}\right|_{M_{1}, M_{2}} . \\
& \text { If } \mathcal{M}=\left(\begin{array}{cc}
\mathcal{M}_{2} & B_{3} \\
B_{3}^{t} & C_{3}
\end{array}\right) \text { is a flat extension of } \mathcal{M}_{2} \text {, then } C_{3}=\left(C_{i j}\right)_{i j} \text { is equal to }\left.W_{1}^{t} \mathcal{M}_{2}\right|_{M_{1}} W_{1} \text { and } \mathcal{M} \text { has a } \\
& \text { moment structure if and only if } \\
& C_{47}=C_{66}, \quad C_{16}=C_{23}, \quad C_{28}=C_{44}, \\
& C_{25}=C_{33}, \quad C_{48}=C_{68}, \quad C_{26}=C_{27}, \\
& C_{12}=C_{13}, \quad C_{14}=C_{22},
\end{aligned}
$$

Proof. The proof is analogous to the proof of Theorem 8.7. Using similar arguments we prove the following claim:

Claim: $C_{18}=C_{24}, C_{16}-C_{23}=C_{46}-C_{38}, C_{37}-C_{27}=C_{13}-C_{12}$.

By Claim the system (8.5) holds if and only if the system (8.58) holds.

We present now a special case which highlights the difference between the classical commutative and the tracial bivariate quartic moment problems with a moment matrix $\mathcal{M}_{2}$ satisfying exactly $\mathbb{Y}^{2}=\mathbb{1}+\mathbb{X}^{2}$. Note that in the case of a cm sequence $\beta$, using a transformation $x \mapsto \frac{x-y}{2}, y \mapsto \frac{x+y}{2}$ gives us a cm sequence $\widetilde{\beta}$ with the moment matrix $\widetilde{\mathcal{M}_{2}}$ satisfying $\mathbb{X} \mathbb{Y}=1$. Therefore, by Theorem 2.7 (3), in the $\mathrm{cm}$ case $\mathcal{M}_{2}$ always admits a flat extension to the moment matrix $\mathcal{M}_{3}$. The following example shows that this is not true in the nc case. However, a nc measure in this example still exists.

Example 8.16. For $\beta_{X^{4}}>\frac{1}{4}$, the following matrices are psd moment matrices of rank 6 satisfying the relation $\mathbb{Y}^{2}=\mathbb{1}+\mathbb{X}^{2}$,

$$
\mathcal{M}_{2}\left(\beta_{X^{4}}\right)=\left(\begin{array}{ccccccc}
1 & 0 & 0 & \frac{1}{2} & 0 & 0 & \frac{3}{2} \\
0 & \frac{1}{2} & 0 & 0 & 0 & 0 & 0 \\
0 & 0 & \frac{3}{2} & 0 & 0 & 0 & 0 \\
\frac{1}{2} & 0 & 0 & \beta_{X^{4}} & 0 & 0 & \frac{1}{2}+\beta_{X^{4}} \\
0 & 0 & 0 & 0 & \frac{1}{2}+\beta_{X^{4}} & 0 & 0 \\
0 & 0 & 0 & 0 & 0 & \frac{1}{2}+\beta_{X^{4}} & 0 \\
\frac{3}{2} & 0 & 0 & \frac{1}{2}+\beta_{X^{4}} & 0 & 0 & 2+\beta_{X^{4}}
\end{array}\right) .
$$

Let us define the moments of degree 5 by (8.56), $B_{3}$ as in Proposition 8.1 and $M_{1}, M_{2}, C_{3}$ as in Theorem 8.15 (2). It is easy to check that

$$
\left(\left.\mathcal{M}_{2}\right|_{M_{1}}\right)^{-1}=\left(\begin{array}{cccc}
\frac{4 \beta_{X^{4}}}{4 \beta_{X^{4}}-1} & 0 & 0 & \frac{2}{1-4 \beta_{X^{4}}} \\
0 & 2 & 0 & 0 \\
0 & 0 & \frac{2}{3} & 0 \\
\frac{2}{1-4 \beta_{X^{4}}} & 0 & 0 & \frac{4}{4 \beta_{X^{4}}-1}
\end{array}\right) \bigoplus\left(\begin{array}{cc}
\frac{2}{1+2 \beta_{X^{4}}} & 0 \\
0 & \frac{2}{1+2 \beta_{X^{4}}}
\end{array}\right)
$$

By a straightworward calculation of $\left(\left.B_{3}\right|_{M_{1}, M_{2}}\right)^{t}\left(\left.\mathcal{M}_{2}\right|_{M_{1}}\right)^{-1}\left(\left.B_{3}\right|_{M_{1}, M_{2}}\right)$ we get that

$$
\begin{aligned}
& C_{47}\left(p, q, \beta_{X^{4}}\right)=\frac{1}{2}+2 \beta_{X^{4}}+2 \beta_{X^{4}}^{2}+\frac{4 q^{2}}{1+2 \beta_{X^{4}}}+\frac{4 p^{2}}{-1+4 \beta_{X^{4}}}, \\
& C_{66}\left(p, q, \beta_{X^{4}}\right)=\frac{4 q^{2}}{1+2 \beta_{X^{4}}}+\frac{4 p^{2}}{-1+4 \beta_{X^{4}}},
\end{aligned}
$$

and hence

$$
\left(C_{47}-C_{66}\right)\left(p, q, \beta_{X^{4}}\right)=\frac{1}{2}\left(1+2 \beta_{X^{4}}\right)^{2} \neq 0 .
$$

Therefore the system from Theorem 8.15 does not have a solution and $\mathcal{M}_{2}\left(\beta_{X^{4}}\right)$ does not admit a flat extension with a moment structure $\mathcal{M}_{3}\left(\beta_{X^{4}}\right)$. However, we will show that for every $\beta_{X^{4}}>\frac{1}{4}, \mathcal{M}_{2}\left(\beta_{X^{4}}\right)$ admits a nc measure. We 
define the matrix function

$$
B(\alpha):=\mathcal{M}_{2}-\alpha\left(\mathcal{M}_{2}^{(0,1)}+\mathcal{M}_{2}^{(0,-1)}\right)=\left(\begin{array}{cccccccc}
1-2 \alpha & 0 & 0 & \frac{1}{2} & 0 & 0 & \frac{3}{2}-2 \alpha \\
0 & \frac{1}{2} & 0 & 0 & 0 & 0 & 0 \\
0 & 0 & \frac{3}{2}-2 \alpha & 0 & 0 & 0 & 0 \\
\frac{1}{2} & 0 & 0 & \beta_{X^{4}} & 0 & 0 & \frac{1}{2}+\beta_{X^{4}} \\
0 & 0 & 0 & 0 & \frac{1}{2}+\beta_{X^{4}} & 0 & 0 \\
0 & 0 & 0 & 0 & 0 & \frac{1}{2}+\beta_{X^{4}} & 0 \\
\frac{3}{2}-2 \alpha & 0 & 0 & \frac{1}{2}+\beta_{X^{4}} & 0 & 0 & 2+\beta_{X^{4}}-2 \alpha
\end{array}\right) .
$$

We have that

$$
\operatorname{det}\left(\left.B(\alpha)\right|_{M_{1}}\right)=\frac{1}{2} \operatorname{det}\left(\left.B(\alpha)\right|_{\left\{1, \mathbb{X}^{2}\right\}}\right)\left(\frac{3}{2}-2 \alpha\right)\left(\frac{1}{2}+\beta_{X^{4}}\right)^{2},
$$

where

$$
\operatorname{det}\left(\left.B(\alpha)\right|_{\left\{\mathbb{1}, \mathbb{X}^{2}\right\}}\right)=\beta_{X^{4}}-\frac{1}{4}-2 \alpha \beta_{X^{4}} .
$$

Let $\alpha_{0}>0$ be the smallest positive number such that the rank of $B\left(\alpha_{0}\right)$ is smaller than 6. By (8.60) and (8.61) we get that

$$
\alpha_{0}=\min \left(\frac{3}{4}, \frac{-1+4 \beta_{X^{4}}}{8 \beta_{X^{4}}}\right) .
$$

For $\beta_{X^{4}}>\frac{1}{4}$ we have that $\alpha_{0}=\frac{-1+4 \beta_{X^{4}}}{8 \beta_{X^{4}}}$. It is easy to check that the kernel of $B\left(\alpha_{0}\right)$ satisfies the relations

$$
\mathbb{X}^{2}=2 \beta_{X^{4}} \mathbb{1}, \quad \mathbb{Y}^{2}=\left(1+2 \beta_{X^{4}}\right) \mathbb{1} .
$$

We also have

$$
\beta_{X}^{(B)}=\beta_{Y}^{(B)}=\beta_{X^{3}}^{(B)}=\beta_{X^{2} Y}^{(B)}=\beta_{X Y^{2}}^{(B)}=\beta_{Y^{3}}^{(B)}=0,
$$

where $\beta_{w(X, Y)}^{(B)}$ are the moments of $B\left(\alpha_{0}\right)$. This is a special case in the proof of Proposition 4.1, i.e., Case 1. Following the proof we see that after using only transformations of type

$$
(x, y) \mapsto\left(\alpha_{1} x+\beta_{1} y, \alpha_{2} x+\beta_{2} y\right)
$$

for some $\alpha_{1}, \alpha_{2}, \beta_{1}, \beta_{2} \in \mathbb{R}$, we come into the basic case 1 of rank 5 with $\widetilde{\beta}_{X}=\widetilde{\beta}_{Y}=\widetilde{\beta}_{X^{3}}=0$. But every such sequence admits a measure of type $(2,1)$ by Theorem 6.5 . Hence $\beta$ admits a measure of type $(4,1)$.

8.5. Relation $\mathbb{Y}^{2}=\mathbb{1}$. The form of $\mathcal{M}_{2}$ is given by the following proposition.

Proposition 8.17. Let $\beta \equiv \beta^{(4)}$ be a nc sequence with a moment matrix $\mathcal{M}_{2}$ satisfying the relation

$$
\mathbb{Y}^{2}=\mathbb{1} .
$$

Then $\mathcal{M}_{2}$ is of the form

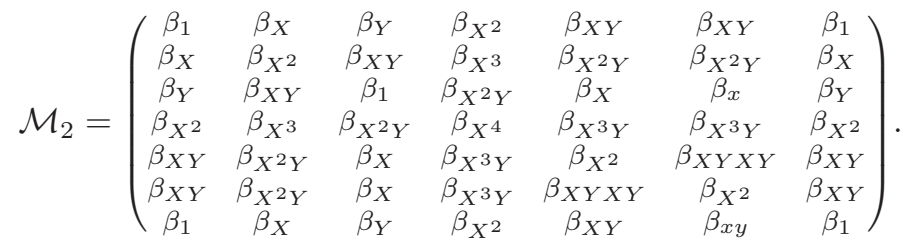

Proof. The relation (8.62) gives us the following system in $\mathcal{M}_{2}$

$$
\begin{array}{rlrl}
\beta_{Y^{2}} & =\beta_{1}, & \beta_{X^{2} Y^{2}}=\beta_{X^{2}}, \\
\beta_{X Y^{2}}=\beta_{X}, & \beta_{X Y^{3}}=\beta_{X Y}, \\
\beta_{Y^{3}}=\beta_{Y}, & \beta_{Y^{4}}=\beta_{Y^{2}} .
\end{array}
$$

This gives the form (8.63) of $\mathcal{M}_{2}$.

The candidate for $B_{3}$ in a moment matrix $\mathcal{M}_{3}$ generated by the measure for $\mathcal{M}_{2}$ is given by the following. 
Proposition 8.18. Let $\beta \equiv \beta^{(4)}$ be a nc sequence with a moment matrix $\mathcal{M}_{2}$ of rank 6 satisfying the relation $\mathbb{Y}^{2}=\mathbb{1}$. Suppose $\beta$ admits a nc measure $\mu$. If $\mathcal{M}_{3}=\left(\begin{array}{cc}\mathcal{M}_{2} & B_{3} \\ B_{3}^{t} & C_{3}\end{array}\right)$ is a moment matrix generated by the measure $\mu$, then $B(3)$ is of the form

$$
B(3)=\left(\begin{array}{cccccccc}
\beta_{X^{3}} & \beta_{X^{2} Y} & \beta_{X^{2} Y} & \beta_{X} & \beta_{X^{2} Y} & \beta_{X} & \beta_{X} & \beta_{Y} \\
\beta_{X^{4}} & \beta_{X^{3} Y} & \beta_{X^{3} Y} & \beta_{X^{2}} & \beta_{X^{4} Y} & \beta_{X Y X Y} & \beta_{X^{2}} & \beta_{X Y} \\
\beta_{X^{3} Y} & \beta_{X^{2}} & \beta_{X Y X Y} & \beta_{X Y} & \beta_{X^{2}} & \beta_{X Y} & \beta_{X Y} & \beta_{1} \\
p & q & q & \beta_{X^{3}} & q & r & \beta_{X^{3}} & \beta_{X^{2} Y} \\
q & \beta_{X^{3}} & r & \beta_{X^{2} Y} & r & \beta_{X^{2} Y} & \beta_{X^{2} Y} & \beta_{X} \\
q & r & r & \beta_{X^{2} Y} & \beta_{X^{3}} & \beta_{X^{2} Y} & \beta_{X^{2} Y} & \beta_{X} \\
\beta_{X^{3}} & \beta_{X^{2} Y} & \beta_{X^{2} Y} & \beta_{X} & \beta_{X^{2} Y} & \beta_{X} & \beta_{X} & \beta_{Y}
\end{array}\right),
$$

where $p, q, r \in \mathbb{R}$ are parameters.

Proof. The RG relations which must hold in $\mathcal{M}_{3}$ are

$$
\mathbb{Y}^{3}=\mathbb{Y}, \quad \mathbb{X} \mathbb{Y}^{2}=\mathbb{X}, \quad \mathbb{Y}^{2} \mathbb{X}=\mathbb{X}
$$

From these relations we get the following system:

$$
\begin{aligned}
\beta_{X^{2} Y^{3}} & =\beta_{X^{2} Y}, & \beta_{Y^{5}} & =\beta_{1}, \\
\beta_{X Y^{4}} & =\beta_{X Y}, & \beta_{X Y^{2} X Y} & =\beta_{X^{2} Y}, \\
\beta_{X^{3} Y^{2}} & =\beta_{X^{3}}, & \beta_{X Y^{4}} & =\beta_{X} .
\end{aligned}
$$

Now the solution of the system (8.66) is given by the statement of the proposition.

Theorem 8.19. Suppose $\beta \equiv \beta^{(4)}$ is a nc sequence with a moment matrix $\mathcal{M}_{2}$ of rank 6 satisfying the relation $\mathbb{Y}^{2}=\mathbb{1}$ and let $B_{3}$ be as in formula (8.65). Then the following are true:

(1) There exists a matrix $W \in \mathbb{R}^{7 \times 10}$ satisfying

$$
B_{3}=\mathcal{M}_{2} W
$$

(2) We write $M_{1}=\left\{\mathbb{1}, \mathbb{X}, \mathbb{Y}, \mathbb{X}^{2}, \mathbb{X} \mathbb{Y}, \mathbb{Y}^{2}\right\}$ and $M_{2}=\left\{\mathbb{X}^{3}, \mathbb{X}^{2} \mathbb{Y}, \mathbb{X} \mathbb{Y}, \mathbb{X} \mathbb{Y}^{2}, \mathbb{Y}^{2}, \mathbb{Y} \mathbb{Y}, \mathbb{Y}^{2}\right\}$. Let $W_{1} \in \mathbb{R}^{6 \times 10}$ be the matrix

If $\mathcal{M}=\left(\begin{array}{cc}\mathcal{M}_{2} & B_{3} \\ B_{3}^{t} & C_{3}\end{array}\right)$ is a flat extension of $\mathcal{M}_{2}$, then $C_{3}=\left(C_{i j}\right)_{i j}$ is equal to $\left.W_{1}^{t} \mathcal{M}_{2}\right|_{M_{1}} W_{1}$ and $\mathcal{M}$ has a moment structure if and only if

$$
\begin{array}{rr}
C_{66}=\beta_{X^{2}} . & C_{16}=C_{23}, \\
C_{25}=C_{33}, & C_{22}=\beta_{X^{4}}, \\
C_{12}=C_{13}, & C_{26}=\beta_{X Y X Y}=\beta_{X^{3} Y} .
\end{array}
$$

Proof. The proof is analogous to the proof of Theorem 8.7. Using similar arguments we prove the following claim:

Claim: $C_{47}=\beta_{X^{2}}, C_{38}=C_{46}, C_{48}=C_{68}, C_{14}=\beta_{X^{4}}, C_{28}=\beta_{44}, C_{27}=\beta_{X^{3} Y}, C_{37}=\beta_{X Y X Y}$.

By Claim the system (8.5) holds if and only if the system (8.67) holds.

We present now a special case which highlights the difference between the classical commutative and the tracial bivariate quartic moment problems with a moment matrix $\mathcal{M}_{2}$ satisfying $\mathbb{Y}^{2}=\mathbb{1}$. By Theorem 2.7 (3), in the cm case $\mathcal{M}_{2}$ always admits a flat extension to the moment matrix $\mathcal{M}_{3}$. The following example shows that this is not true in the nc case. However, a nc measure in this example still exists.

Example 8.20. For $\beta_{X^{4}}>1$, the following matrices are psd moment matrices of rank 6 satisfying $\mathbb{Y}^{2}=\mathbb{1}$,

$$
\mathcal{M}_{2}\left(\beta_{X^{4}}\right)=\left(\begin{array}{ccccccc}
1 & 0 & 0 & 1 & 0 & 0 & 1 \\
0 & 1 & 0 & 0 & 0 & 0 & 0 \\
0 & 0 & 1 & 0 & 0 & 0 & 0 \\
1 & 0 & 0 & \beta_{X^{4}} & 0 & 0 & 1 \\
0 & 0 & 0 & 0 & 1 & 0 & 0 \\
0 & 0 & 0 & 0 & 0 & 1 & 0 \\
1 & 0 & 0 & 1 & 0 & 0 & 1
\end{array}\right) .
$$


Let us define $B_{3}$ as in Proposition 8.1 and $M_{1}, M_{2}, C_{3}$ as in Theorem 8.19 (2). It is easy to check that

$$
\left(\left.\mathcal{M}_{2}\right|_{M_{1}}\right)^{-1}=\left(\begin{array}{cccc}
\frac{\beta_{X^{4}}}{\beta_{X^{4}}-1} & 0 & 0 & \frac{1}{1-\beta_{X^{4}}} \\
0 & 1 & 0 & 0 \\
0 & 0 & 1 & 0 \\
\frac{1}{1-\beta_{X^{4}}} & 0 & 0 & \frac{1}{\beta_{X^{4}}-1}
\end{array}\right) \bigoplus\left(\begin{array}{ll}
1 & 0 \\
0 & 1
\end{array}\right) .
$$

By a straightworward calculation of $\left(\left.B_{3}\right|_{M_{1}, M_{2}}\right)^{t}\left(\left.\mathcal{M}_{2}\right|_{M_{1}}\right)^{-1}\left(\left.B_{3}\right|_{M_{1}, M_{2}}\right)$ we get that

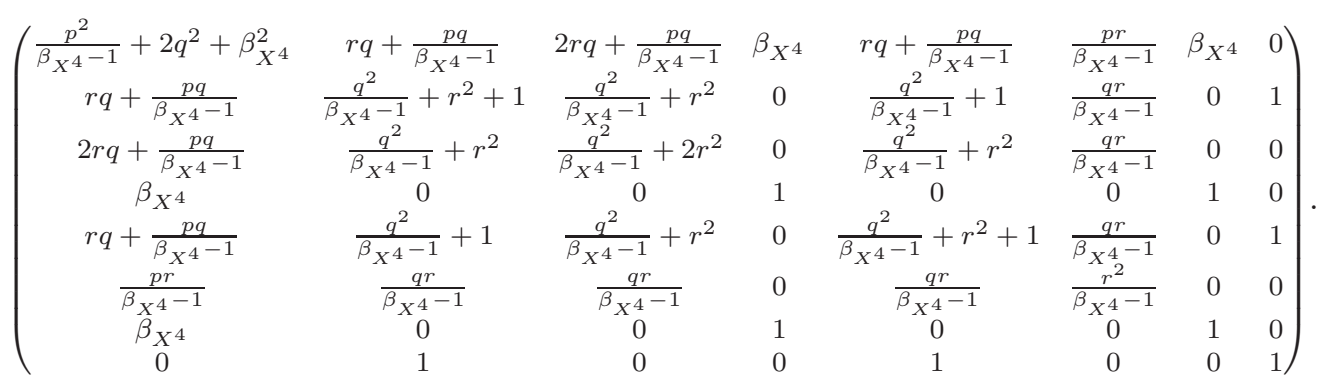

For the flat extension we must have, by Theorem 8.19,

$$
\begin{aligned}
\frac{r^{2}}{\beta_{X^{4}}-1}=1, & \frac{p r}{\beta_{X^{4}}-1}=\frac{q^{2}}{\beta_{X^{4}}-1}+r^{2}, \\
\frac{q^{2}}{\beta_{X^{4}}-1}+1=\frac{q^{2}}{\beta_{X^{4}}-1}+2 r^{2}, & \frac{q^{2}}{\beta_{X^{4}}-1}+r^{2}+1=\beta_{X^{4}}, \\
r q+\frac{p q}{\beta_{X^{4}}-1}=2 r q+\frac{p q}{\beta_{X^{4}}-1}, & \frac{q r}{\beta_{X^{4}}-1}=0 .
\end{aligned}
$$

It is easy to see these equations are satisfied if and only if

$$
\beta_{X^{4}}=\frac{3}{2} \quad \text { in which case } \quad p= \pm \frac{1}{2 \sqrt{2}}, \quad q=0, \quad r= \pm \frac{1}{\sqrt{2}} .
$$

However, we will prove that for every $\beta_{X^{4}}>1, \mathcal{M}_{2}\left(\beta_{X^{4}}\right)$ admits a nc measure. We define the matrix function

$$
B(\alpha):=\mathcal{M}_{2}\left(\beta_{X^{4}}\right)-\alpha A,
$$

where

$$
A=\left(\begin{array}{ccccccc}
1 & 0 & 0 & 1 & 0 & 0 & 1 \\
0 & 1 & 0 & 0 & 0 & 0 & 0 \\
0 & 0 & 1 & 0 & 0 & 0 & 0 \\
1 & 0 & 0 & 1 & 0 & 0 & 1 \\
0 & 0 & 0 & 0 & 1 & -1 & 0 \\
0 & 0 & 0 & 0 & -1 & 1 & 0 \\
1 & 0 & 0 & 1 & 0 & 0 & 1
\end{array}\right)
$$

$A$ is a psd moment matrix of rank 4 satisfying the relations

$$
\mathbb{X}^{2}=\mathbb{1}, \quad \mathbb{X} \mathbb{Y}+\mathbb{Y} \mathbb{X}=\mathbf{0}, \quad \mathbb{Y}^{2}=\mathbb{1}
$$

and thus admits a nc measure by Theorem 3.1 (3). But then

$$
B\left(\frac{1}{2}\right)=\left(\begin{array}{ccccccc}
\frac{1}{2} & 0 & 0 & \frac{1}{2} & 0 & 0 & \frac{1}{2} \\
0 & \frac{1}{2} & 0 & 0 & 0 & 0 & 0 \\
0 & 0 & \frac{1}{2} & 0 & 0 & 0 & 0 \\
\frac{1}{2} & 0 & 0 & \beta_{X^{4}}-\frac{1}{2} & 0 & 0 & \frac{1}{2} \\
0 & 0 & 0 & 0 & \frac{1}{2} & \frac{1}{2} & 0 \\
0 & 0 & 0 & 0 & \frac{1}{2} & \frac{1}{2} & 0 \\
\frac{1}{2} & 0 & 0 & \frac{1}{2} & 0 & 0 & \frac{1}{2}
\end{array}\right)
$$

is a psd $\mathrm{cm}$ moment matrix of rank 5 satisfying $\mathbb{Y}^{2}=\mathbb{1}$ and $\mathbb{X} \mathbb{Y}=\mathbb{Y} \mathbb{X}$ and hence admits a 5 -atomic measure with the atoms of the from $\left(x_{j}, y_{j}\right) \in \mathbb{R}^{2}, j=1, \ldots, 5$, by Theorem 2.7. 


\section{REFERENCES}

[Akh65] Akhiezer, N.I.: The classical moment problem and some related questions in analysis. Hafner Publishing Co., New York (1965)

[AV03] Ambrozie, C.G., Vasilescu, F.H.: Operator-theoretic Positivstellensätze. Z. Anal. Anwend. 22, 299-314 (2003)

[BW11] Bakonyi, M., Woerdeman, H.J.: Matrix Completions, Moments, and Sums of Hermitian Squares. Princeton University Press, Princeton (2011)

[BT06] Bayer, C., Teichmann, J.: The proof of Tchakaloff's theorem. Proc. Amer. Math. Soc. 134, 3035-3040 (2006)

[BMV75] Bessis, D., Moussa, P., Villani, M.: Monotonic converging variational approximations to the functional integrals in quantum statistical mechanics. J. Math. Phys. 16, 2318-2325 (1975)

[Bha16] Bhardwaj, A.: Trace Positive, Non-commutative Polynomials and the Truncated Moment Problem, MSc Thesis, University of Auckland, Auckland, https://researchspace.auckland.ac.nz/handle/2292/30249, (2016)

[Bur11] Burgdorf, S.: Sums of hermitian squares as an approach to the BMV conjecture. Linear and Multilinear Algebra 59, 1-9 (2011)

[BCKP13] Burgdorf, S., Cafuta, K., Klep, I., Povh, J.: The tracial moment problem and trace-optimization of polynomials. Math. Program. 137, 557-578 (2013)

[BK10] Burgdorf, S., Klep, I.: Trace-positive polynomials and the quartic tracial moment problem. C. R. Math. Acad. Sci. Paris 348, 721-726 (2010)

[BK12] Burgdorf, S., Klep, I.: The truncated tracial moment problem. J. Oper. Theory 68, 141-163 (2012)

[BKP16] Burgdorf, S., Klep, I., Povh, J.: Optimization of polynomials in non-commuting variables. SpringerBriefs in Mathematics, Springer-Verlag (2016)

[Caf13] Cafuta, K.: On matrix algebras associated to sum-of-squares semidefinite programs, Linear Multilinear Algebra 61 (2013) $1496-1509$.

[CMN11] Cimprič, J., Marshall, M., Netzer, T.: On the real multidimensional rational $K$-moment problem. Trans. Amer. Math. Soc. 363, 5773$5788(2011)$

[CZ12] Cimprič, J., Zalar, A.: Moment problems for operator polynomials. J. Math. Anal. Appl. 401, 307-316 (2013)

[Con76] Connes, A.: Classification of injective factors. Cases $I I_{1}, I I_{\infty}, I I I_{\lambda}, \lambda \neq 1$. Ann. Math. 104, 73-115 (1976)

[CF96] Curto, R., Fialkow, L.: Solution of the truncated complex moment problem for flat data. Mem. Amer. Math. Soc. 119, no. 568 (1996)

[CF98a] Curto, R., Fialkow, L.: Flat extensions of positive moment matrices: relations in analytic or conjugate terms. Oper. Theory Adv. Appl. 104 59-82 (1998)

[CF98b] Curto, R., Fialkow, L.: Flat extensions of positive moment matrices: recursively generated relations. Mem. Amer. Math. Soc. 136, no. 648 (1998)

[CF02] Curto, R., Fialkow, L.: Solution of the singular quartic moment problem. J. Operator Theory 48, 315-354 (2002)

[CF04] Curto, R., Fialkow, L.: Solution of the truncated parabolic moment problem. Integral Equations Operator Theory 50, 169-196 (2004)

[CF05] Curto, R., Fialkow, L.: Solution of the truncated hyperbolic moment problem. Integral Equations Operator Theory 52, 181-218 (2005)

[CF08] Curto, R., Fialkow, L.: An analogue of the Riesz-Haviland theorem for the truncated moment problem. J. Funct. Anal. 225, 2709-2731 (2008)

[CS16] Curto, R., Seonguk, Y.: Concrete solution to the nonsingular quartic binary moment problem. Proc. Amer. Math. Soc. 144, 249-258 (2016)

[DP01] Delzell, C.N., Prestel, A.: Positive polynomials. From Hilbert's 17th problem to real algebra. Springer Monogr. Math., (2001)

[DLTW08] Doherty, A.C., Liang, Y.-C., Toner, B., Wehner, S.: The quantum moment problem and bounds on entangled multi-prover games. In: Twenty-Third Annual IEEE Conference on Computational Complexity, pp. 199-210. IEEE Computer Soc., Los Alamitos, CA (2008)

[Hav35] Haviland, E.K.: On the momentum problem for distribution functions in more than one dimension II. Amer. J. Math. 58, 164-168 (2006)

[FN10] Fialkow, L., Nie, J.: Positivity of Riesz functionals and solutions of quadratic and quartic moment problems. J. Funct. An. 258, 328-356 (2010)

[Fia14] Fialkow, L.: The truncated moment problem on parallel lines. The Varied Landscape of Operator Theory, 99-116 (2014)

[GKM16] Ghasemi, M., Kuhlmann, S., Marshall, M.: Moment problem in infinitely many variables. Israel J. Math. 212, 989-1012 (2016)

[Hel02] Helton, J.W.: "Positive" non-commutative polynomials are sums of squares. Ann. of Math. 156, 675-694 (2002)

[HKM12] Helton, J.W., Klep, I., McCullough, S.: The convex Positivstellensatz in a free algebra. Adv. Math. 231, 516-534 (2012)

[HM04] Helton, J.W., McCullough, S.: A Positivstellensatz for noncommutative polynomials. Trans. Amer. Math. Soc. 365, 3721-3737 (2004)

[IKR14] Infusino, M., Kuna, T., Rota, A.: The full infinite dimensional moment problem on semialgebraic sets of generalized functions. J. Funct. Analysis 267, 1382-1418 (2014)

[KW13] Kimsey, D.P., Woerdeman, H.J.: The multivariable matrix valued $K$-moment problem on $\mathbb{R}^{d}, \mathbb{C}^{d}, \mathbb{T}^{d}$. Trans. Amer. Math. Soc. 365, 5393-5430 (2013).

[Kov83] Kovalishina, I.V.: Analytic theory of a class of interpolation problems. Izv. Akad. Nauk SSSR Ser. Mat. 47, 455-497 (1983)

[Kre49] Krein, M.: Infinite J-matrices and a matrix-moment problem. Doklady Akad. Nauk SSSR (N.S.) 69, 125-128 (1949)

[KN77] Krein, M.G., Nudelman, A.A.: The Markov moment problem and extremal problems. Translations of Mathematical Monographs, Amer. Math. Soc., (1977)

[KM02] Kuhlmann, S., Marshall, M.: Positivity, sums of squares and the multidimensional moment problem. Trans. Amer. Math. Soc. 354, 4285-4301 (2002)

[KS08a] Klep, I., Schweighofer, M.: Connes' embedding conjecture and sums of hermitian squares. Adv. Math. 217, 1816-1837 (2008)

[KS08b] Klep, I., Schweighofer, M.: Sums of hermitian squares and the BMV conjecture. J. Stat. Phys. 133, 739-760 (2008)

[Las09] Lasserre, J.B.: Moments, positive polynomials and their applications. Imperial College Press, (2009)

[Lau05] Laurent, M.: Revising two theorems of Curto and Fialkow on moment matrices. Proc. Amer. Math. Soc. 133, 2965-2976 (2005)

[Lau09] Laurent, M.: Sums of squares, moment matrices and optimization over polynomials. In: Emerging Applications of Algebraic Geometry, Vol. 149 of IMA Volumes in Mathematics and its Applications, pp. 157-270, Springer-Verlag, (2009)

[LP15] Laurent, M., Piovesan, T.: Conic approach to quantum graph parameters using linear optimization over the completely positive semidefinite cone. SIAM J. Optim. 25, 2461-2493 (2015) 
[JP01] Jacobi, T., Prestel, A.: Distinguished representations of strictly positive polynomials. J. Reine Angew. Math. 532, 223-235 (2001)

[Mar08] Marshall, M.: Positive polynomials and sums of squares. Mathematical Surveys and Monographs 146, Amer. Math. Soc., (2008)

[McC01] McCullough, S.: Factorization of operator-valued polynomials in several non-commuting variables. Linear Algebra Appl. 326, 193-204 (2001)

[PS01] Powers, V., Scheiderer, C.: The moment problem for non-compact semialgebraic sets. Adv. Geom. 1, 71-88 (2001)

[Put93] Putinar, M.: Positive polynomials on compact semi-algebraic sets. Indiana Univ. Math. J. 42, 969-984 (1993)

[PS06] Putinar, M., Scheiderer, C.: Multivariate moment problems: Geometry and indeterminateness. Ann. Sc. Norm. Super. Pisa Cl. Sci. 5, 137-157 (2006)

[PS08] Putinar, M., Schmüdgen, K.: Multivariate determinateness. Indiana Univ. Math. J. 57, 2931-2968 (2008)

[PV99] Putinar, M., Vasilescu, F.-H.: Solving moment problems by dimensional extension. Ann. of Math. 149, 1087-1107 (1999)

[Qua15] Quarez, R.: Trace-positive non-commutative polynomials. Proc. Amer. Math. Soc. 143, 3357-3370 (2015)

[Sch91] Schmüdgen, K.: The K-moment problem for compact semi-algebraic sets. Math. Ann. 289, 203-206 (1991)

[Smu59] Smul'jan, J.L.: An operator Hellinger integral, Mat. Sb. (N.S.) 49, 381-430 (1959)

[Sto01] Stochel, J.: Solving the truncated moment problem solves the moment problem/ Glasgow J. Math. 43, 335- 341 (2001)

[Tch57] Tchakaloff, V.: Formules de cubatures mécaniques à coefficients non négatifs. Bull. Sci. Math. 81, 123-134 (1957)

[Vas03] Vasilescu, F.H.: Spectral measures and moment problems. In: Spectral theory and its applications, pp. 173-215 (2003)

[Wol] Wolfram Research, Inc., Mathematica, Version 9.0, Wolfram Research, Inc., Champaign, IL (2012) 